University of South Florida

DIGITAL COMMONS

Digital Commons @ University of

@ UNIVERSITY OF SOUTH FLORIDA

South Florida

School of Geosciences Faculty and Staff

Publications

School of Geosciences

4-2002

\title{
REVEL: A Model for Recent Plate Velocities from Space Geodesy
}

Giovanni F. Sella

Louisiana State University

Timothy H. Dixon

University of Miami, thd@usf.edu

Ailin Mao

University of Miami

Follow this and additional works at: https://digitalcommons.usf.edu/geo_facpub

Part of the Earth Sciences Commons

\section{Scholar Commons Citation}

Sella, Giovanni F.; Dixon, Timothy H.; and Mao, Ailin, "REVEL: A Model for Recent Plate Velocities from Space Geodesy" (2002). School of Geosciences Faculty and Staff Publications. 468.

https://digitalcommons.usf.edu/geo_facpub/468

This Article is brought to you for free and open access by the School of Geosciences at Digital Commons @ University of South Florida. It has been accepted for inclusion in School of Geosciences Faculty and Staff Publications by an authorized administrator of Digital Commons @ University of South Florida. For more information, please contact digitalcommons@usf.edu. 


\title{
REVEL: A model for Recent plate velocities from space geodesy
}

\author{
Giovanni F. Sella ${ }^{1}$ \\ Department of Geology and Geophysics, Louisiana State University, Baton Rouge, Louisiana
}

\author{
Timothy H. Dixon and Ailin $\mathrm{Mao}^{2}$ \\ Rosenstiel School of Marine and Atmospheric Science, University of Miami, Miami, Florida
}

Received 30 October 2000; revised 21 January 2002; accepted 31 January 2002; published 27 April 2002.

[1] We present a new global model for Recent plate velocities, REVEL, describing the relative velocities of 19 plates and continental blocks. The model is derived from publicly available space geodetic (primarily GPS) data for the period 1993-2000. We include an independent and rigorous estimate for GPS velocity uncertainties to assess plate rigidity and propagate these uncertainties to the velocity estimates. The velocity fields for North America, Eurasia, and Antarctica clearly show the effects of glacial isostatic adjustment, and Australia appears to depart from rigid plate behavior in a manner consistent with the mapped intraplate stress field. Two thirds of tested plate pairs agree with the NUVEL-1A geologic (3 Myr average) velocities within uncertainties. Three plate pairs (Caribbean-North America, Caribbean-South America, and North America-Pacific) exhibit significant differences between the geodetic and geologic model that may reflect systematic errors in NUVEL-1A due to the use of seafloor magnetic rate data that do not reflect the full plate rate because of tectonic complexities. Most other differences probably reflect real velocity changes over the last few million years. Several plate pairs (Arabia-Eurasia, Arabia-Nubia, Eurasia-India) move more slowly than the 3 Myr NUVEL-1A average, perhaps reflecting long-term deceleration associated with continental collision. Several other plate pairs, including Nazca-Pacific, Nazca-South America and Nubia-South America, are experiencing slowing that began $\sim 25 \mathrm{Ma}$, the beginning of the current phase of Andean crustal shortening. INDEX TERMS: 1243 Geodesy and Gravity: Space geodetic surveys; 8107 Tectonophysics: Continental neotectonics; 8150 Evolution of the Earth: Plate boundary - general (3040); 8158 Evolution of the Earth: Plate motions - present and recent (3040); KEYWORDS: Plate tectonics, geodesy, GPS, global plate model, present-day, REVEL

\section{Introduction}

[2] The present-day velocities of the Earth's lithospheric plates are an important kinematic boundary condition for many geologic and geophysical studies, including regional neotectonics, seismogenic zone processes, and earthquake hazards. Currently, the most comprehensive picture of geologically young plate motion comes from the global geologic model NUVEL-1A [DeMets et al., 1990, 1994], a significant update of earlier global models [Chase, 1972, 1978; Minster et al., 1974; Minster and Jordan, 1978]. NUVEL-1A is based in large part on mid-ocean ridge spreading rates dated from magnetic anomaly $2 \mathrm{~A}(\sim 3 \mathrm{Ma}$, or mid-Pliocene time) and thus describes relative plate velocities averaged over Pliocene to Recent time. NUVEL-1A is a robust model based on a large data set, but it nevertheless has some deficiencies. First, some smaller plates are necessarily omitted because of lack of data. Second, the geologic model may be biased due to poor or insufficient kinematic data, e.g., North America-Pacific relative motion [DeMets, 1995; DeMets and Dixon, 1999] and motion of the Caribbean plate relative to North and South America [Dixon et al., 1998; DeMets et al., 2000; Weber et al., 2001; Perez et al., 2001]. Third, the 3-Myr average velocity predicted by NUVEL$1 \mathrm{~A}$ or any other geologic model may yield biased estimates of

\footnotetext{
${ }^{1}$ Now at Department of Geological Sciences, Northwestern University, Evanston, Illinois.

${ }^{2}$ Also at Magellan Systems, San Dimas, California.

present-day velocity for some plate pairs because the plates are speeding up, slowing down, or changing direction, e.g., NazcaSouth America [Norabuena et al., 1998, 1999; Angermann et al., 1999].

[3] Space geodesy has the potential to measure relative plate velocities directly over periods of just a few years, as demonstrated by satellite laser ranging (SLR) [Smith et al., 1990; Robbins et al., 1993; Cazenave et al., 1993; Sengoku, 1998], very long baseline interferometry (VLBI) [Argus and Gordon, 1990; Robaudo and Harrison, 1993; Ryan et al., 1993; Sato, 1993]; Doppler Orbitography and Radiopositioning Integrated by Satellite (DORIS) [Cazenave et al., 1992; Soudarin and Cazenave, 1993, 1995; Cretaux et al., 1998] and the Global Positioning System (GPS) [Dixon et al., 1991a; Dixon, 1993; Argus and Heflin, 1995; Larson et al., 1997; Dixon and Mao, 1997]. Most studies to date suggest that the great majority of plate velocities estimated from space geodesy are consistent with the NUVEL-1A model within $95 \%$ confidence. However, uncertainties in the geodetic estimates have been large enough that important differences may have been missed. The true uncertainty of space geodetic data has also been difficult to quantify. Therefore it has been difficult to address an important tectonophysical problem, namely, the extent to which individual plate velocities may be changing over the last few million years and to what extent such changes, if they occur, can be understood in terms of simple plate-driving forces.

[4] New space geodetic data and new analytical techniques now permit a significant refinement of our description of presentday plate motion. In this paper we present a comprehensive velocity model for most major and several minor plates and 
continental blocks, based primarily on GPS. Our study differs from previous global studies in several respects:

1. A very large geodetic data set is now publicly available through the efforts of many individuals, institutions, and geodetic agencies, permitting a more accurate and more comprehensive geodetic plate motion model. This data set represents both a large number of sites, giving generally good geographic distribution, and long, nearly continuous time series at many individual sites, resulting in precise site velocity estimates. Most major plates now have at least two GPS sites, the minimum number to determine a plate's angular velocity with space geodesy.

2. We include velocity estimates for the Amuria, Anatolia, Caribbean, Nubia, Okhotsk, Philippine, Sierra Nevada, Somalia, South China, and Sunda plates or continental blocks. While these and other plates and continental blocks have been the subject of earlier local studies, most previous global geologic and geodetic plate motion models have omitted one or more of these plates because of sparse data or have approximated Nubia (west Africa) by combining it with Somalia (east Africa).

3. We incorporate a rigorous, independent estimate for GPS velocity errors. This permits simple, objective tests of whether the GPS site velocity for a given location is consistent with rigid plate behavior and whether plate motions averaged over the last few years differ significantly from motions averaged over the last few million years.

[5] The velocity predictions of geologic plate motion models are sometimes termed "present-day" or "current" because they describe geologically young plate motion, derived from the youngest easily identified magnetic anomaly, typically $2 \mathrm{~A}(\sim 3 \mathrm{Ma}$, or mid-Pliocene) [Minster and Jordan, 1978; DeMets et al., 1990, $1994]$ to, in some cases, anomaly $1 \mathrm{~N}(\sim 0.8 \mathrm{Ma}$, or mid-Pleistocene) [DeMets, 1995; Conder and Forsyth, 2000]. Our geodetic plate motion model is derived from data over a very different time span, roughly the last decade. It is probably representative of plate motions over the Holocene or Recent epoch (last $\sim 10,000$ years) and possibly the late Pleistocene epoch (last few hundred thousand years) provided that we account for, or avoid, short-term strain effects related to the seismic cycle and isostatic effects associated with the last glacial cycle. The former can impact site velocities in the vicinity of active plate boundary zone faults, while the latter may impact site velocities in parts of North America, Eurasia, Greenland, and Antarctica. To emphasize the time span over which we believe our model to be valid, we have termed it "REVEL" (for Recent velocities) with the suffix 2000, to indicate the last year of data included in the model. We expect that the model can be improved significantly in subsequent years as additional data are added and time series lengthen.

\section{Data Analysis}

[6] Uncertainty in the positions of the GPS satellites is a major error source for the coordinate time series used in this study. By 1993 the global tracking network for GPS satellites became sufficiently robust to produce more accurate satellite ephemerides (satellite positions as a function of time) compared to earlier periods. Our data span the time period 1 January 1993 to 31 December 2000. To ensure consistency, all data were reanalyzed specifically for this study, resulting in a uniform set of site velocity and error estimates. The great majority of sites used in this study are continuous, in the sense that they record data for 20-24 hours per day, typically for at least 300 days per year (Table 1). For more than $98 \%$ of the continuous sites presented here, all known existing data acquired after 1 January 1993 were processed (for the remaining sites, there may be additional data that were not available to us). In some cases (the Caribbean and Philippine plates and the Sierra Nevada block), continuous site distribution is limited, and we have augmented these data with data from "episodic" sites occupied periodically, usually every year or two. A total of $\sim 345,000$ station days of data were analyzed for this study, most of which lie in stable plate interiors and are used to estimate plate velocities (Table 1). The data were analyzed at the University of Miami, generally following procedures outlined by Dixon et al. [1993, 1997], although some of these procedures have been updated considerably. Salient points are listed here:

1. We use GIPSY/OASIS II, Release 5.0 software developed at the Jet Propulsion Laboratory (JPL) and nonfiducial satellite orbit and clock files provided by JPL [Zumberge et al., 1997].

2. We use the ionosphere-free combination of both undifferenced carrier phase and $\mathrm{P}$ code pseudorange data (data weights of 1 $\mathrm{cm}$ and $1 \mathrm{~m}$, respectively), typically recorded at 30-s intervals, with a 5-min decimation rate.

3. We created a comprehensive antenna height and type change file going back to 1993 for all sites, and used the relative dome-free models of Mader [1999] for first-order antenna phase center corrections.

4. To improve the definition of horizontal atmospheric gradients and to reduce their effect on the final position estimates, we use an elevation angle cutoff of $10^{\circ}$ (where data are available) and the horizontal gradient model of Bar-Sever et al. [1998]. We use the same random walk model for the zenith delay as Bar-Sever et al. $(3 \mathrm{~mm} / \sqrt{ } \mathrm{h})$, but a looser constraint for the two orthogonal horizontal gradients $(5 \mathrm{~mm} / \sqrt{ } \mathrm{h})$. We use the mapping function of Niell [1996], which describes how the average atmospheric path delay varies as a function of elevation angle.

5. All sites are corrected for ocean tidal loading, using the NLOADF program of Agnew [1997], and the Schwiderski [1980] ocean tide model.

6. Carrier phase cycle ambiguities are estimated, not fixed.

7. We estimated offset parameters at the date of each change of antenna height or model to correct for second-order effects such as inaccuracy of phase center estimates and incorrect records of antenna height changes; these can exceed $1 \mathrm{~cm}$ in the vertical component, but generally are a few millimeters or less for horizontal components. Offsets were also calculated for antenna dome changes.

8. Daily position estimates are generated with loose constraints [Heflin et al., 1992; Blewitt et al., 1992], then transformed to International Terrestrial Reference Frame (ITRF)-97 [Boucher et al., 1999] using up to 51 colocated sites whose positions are defined in ITRF-97. The number of colocated sites depends on station availability. For 1998 and later, this usually exceeds 45, but in 1993, for example, it was typically 15-20. Transformation parameters are computed each day; hence each daily position estimate is essentially independent.

9. Position estimates with formal errors $>9.9 \mathrm{~m}$ are removed from the database (typically $<2$ days per site per year).

[7] Velocity estimates are based on a weighted least squares line fit to the daily position estimates, including the offset parameters described above (Figure 1). Outliers, defined as points that lie off the best fit line by more than 3 times the formal error, are flagged, but not removed from the database, and are not used in the line fit (the fitting and outlier definition are done iteratively). Sites listed in upper case letters in Table 1 and in figures (e.g., STJO) lie on the stable plate and are used to define the rigid plate angular velocity. Sites in lowercase (e.g., cic*, algo) are not used in the rigid plate definition because they may lie in the deforming boundary zone or may be affected by glacial isostatic adjustment or other nonrigid process. In some cases a station was moved or offset during the 1993-2000 period of this study. Where possible, we used publicly available vector tie information ("site ties") to link the two time series. If vector tie information was unavailable, we used a procedure similar to that described in point 7 above to tie the two time series together, in effect estimating the vector tie along with the slope (velocity) parameter of interest (Figure 1 shows an example). In such cases we are estimating three parameters (slope, intercept, and offset) rather 


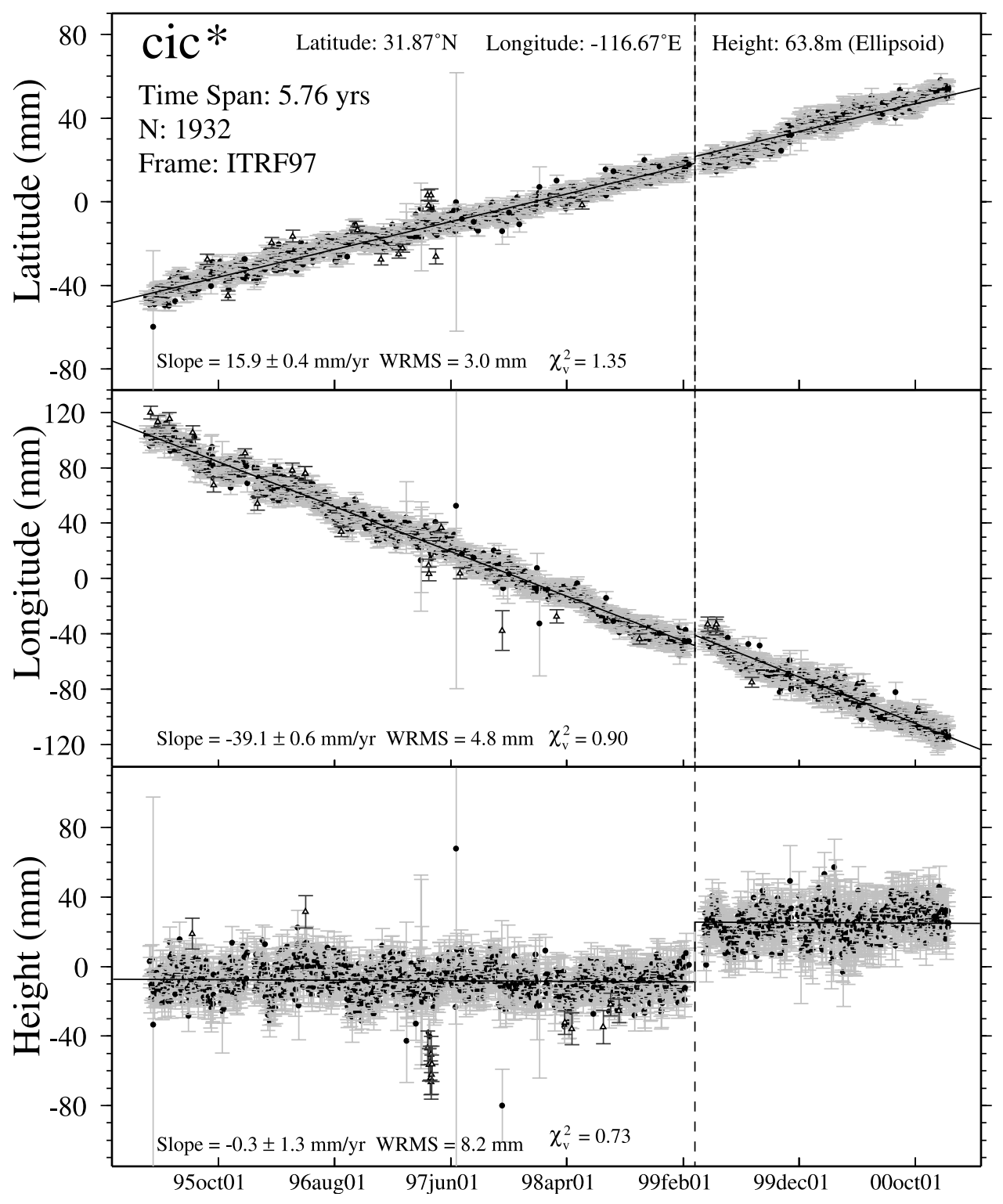

Figure 1. Example coordinate time series for site cic* (Table 1), a composite time series composed of Cice prior to 1 March 1999 and Cic1 thereafter. Note offset estimate at date of site change (vertical dashed line) after site tie correction. Site velocity is given by slope of weighted least squares line fit (thin solid line) through the data (solid circles), excluding outliers (open triangles). Site velocity error is the uncertainty of the slope estimate, accounting for uncorrelated and time-correlated noise, total time span of observations, and total number of observations.

than the normal two parameters for a straight line. This allows us to take advantage of the reduction of velocity uncertainty associated with longer time series. Site names for such linked time series are indicated by asterisks in Table 1 and in the figures; e.g., cic* represents the combination of time series from sites Cice and Cic1 (Figure 1). Velocities for the original sites are also listed in Table 1 immediately preceding the combined solution with only the first letter capitalized. In a few cases, time series have obvious offsets, presumably associated with an equipment or other significant change, even though no change is reported. In cases where such offsets are large enough to affect the velocity significantly, we have estimated the offset following the procedure outlined above. These sites are noted in Table 1 .

[8] Our velocity error estimates account for both white (uncorrelated) and colored (time-correlated) noise [Langbein and Johnson, 1997] and are modeled following Mao et al. [1999]. This approach is based on a numerical analysis of 23 globally distributed GPS sites with time series spanning the period 1994-1997. The error model avoids any assumptions concerning the fit of GPS data to a particular geophysical model (e.g., rigid plate model) and thus provides independent error estimates. To account for improvements in analytical techniques since 1997 and for site-specific effects, we exploit the correlation between WRMS (the weighted root mean square scatter of the daily position estimates about a best fit straight line) and white and flicker noise amplitudes observed in the data of Mao et al. [1999], as outlined by Dixon et al. [2000a]. Random walk noise is assumed to be zero. When this error model is applied to our data set of 64 site velocities from the stable interior of North America, we obtain a $\chi^{2}$ per degree of freedom of 1.05 for the rigid plate model, close to the expected value of 1.0. This suggests that the error model is reasonable and that the region sampled by these data can be assumed rigid within data uncertainty. This is discussed in more detail in section 4.1. 
Table 1. Site Positions and Velocities Relative to ITRF-97

\begin{tabular}{|c|c|c|c|c|c|c|c|c|c|c|c|c|}
\hline \multirow[t]{2}{*}{ Site $^{\mathrm{a}}$} & \multicolumn{2}{|c|}{ Position $^{\mathrm{b}}$} & \multirow{2}{*}{$\begin{array}{l}\Delta T_{,}^{\mathrm{c}} \\
\text { years }\end{array}$} & \multirow{2}{*}{$\begin{array}{l}\text { Total } \\
\text { Data }^{\mathrm{d}}\end{array}$} & \multicolumn{3}{|c|}{ Velocity $^{\mathrm{e}}$} & \multicolumn{3}{|c|}{ WRMS $^{\mathrm{f}}$} & \multicolumn{2}{|c|}{ Rate Res. ${ }^{g}$} \\
\hline & ${ }^{\circ} \mathrm{N}$ & ${ }^{\circ} \mathrm{E}$ & & & North & East & Vertical & $\mathrm{N}$ & E & V & $\mathrm{N}$ & E \\
\hline \multicolumn{13}{|l|}{ Amuria (Am) } \\
\hline Taej $^{\text {h }}$ & 36.37 & 127.37 & 3.32 & 1185 & $-17.8 \pm 0.9$ & $27.9 \pm 1.2$ & $3.8 \pm 2.7$ & 3.7 & 5.9 & 11.3 & - & - \\
\hline Daej ${ }^{\mathrm{h}}$ & 36.40 & 127.37 & 1.79 & 619 & $-12.4 \pm 1.6$ & $25.6 \pm 2.1$ & $-1.0 \pm 4.8$ & 3.5 & 5.3 & 10.2 & - & - \\
\hline $\mathrm{DAE}^{* \mathrm{i}}$ & 36.40 & 127.37 & 5.12 & 1804 & $-17.0 \pm 0.6$ & $27.6 \pm 0.8$ & $3.0 \pm 1.8$ & 4.0 & 5.8 & 11.1 & -1.1 & -0.2 \\
\hline SUWN & 37.28 & 127.05 & 3.07 & 920 & $-14.2 \pm 0.9$ & $27.8 \pm 1.2$ & $1.9 \pm 2.7$ & 3.2 & 5.2 & 9.2 & 1.7 & 0.2 \\
\hline VLAD & 43.20 & 131.93 & 4.47 & 1363 & $-15.7 \pm 0.8$ & $24.8 \pm 0.8$ & $-1.5 \pm 2.0$ & 4.4 & 5.4 & 11.0 & 0.3 & 0.1 \\
\hline bjfs & 39.61 & 115.89 & 1.20 & 419 & $-13.8 \pm 2.4$ & $29.9 \pm 2.8$ & $-3.9 \pm 7.5$ & 3.4 & 4.9 & 11.0 & 1.3 & 1.1 \\
\hline irkt & 52.22 & 104.32 & 5.27 & 1867 & $-9.1 \pm 0.7$ & $26.5 \pm 0.7$ & $-1.8 \pm 1.7$ & 4.3 & 5.4 & 10.6 & 4.6 & -0.3 \\
\hline xian & 34.37 & 109.22 & 3.48 & 991 & $-14.7 \pm 0.9$ & $34.5 \pm 1.1$ & $0.1 \pm 2.5$ & 3.8 & 5.2 & 10.0 & -0.4 & 3.4 \\
\hline \multicolumn{13}{|l|}{ Anatolia (At) } \\
\hline Ankr ${ }^{\mathrm{h}}$ & 39.89 & 32.76 & 5.52 & 1580 & $10.3 \pm 0.7$ & $0.9 \pm 1.0$ & $-0.9 \pm 1.5$ & 4.9 & 7.5 & 9.4 & 0.0 & 0.0 \\
\hline $\mathrm{ANK}^{* \mathrm{j}}$ & 39.89 & 32.76 & 4.14 & 1280 & $8.1 \pm 0.7$ & $4.8 \pm 0.7$ & $-3.7 \pm 1.9$ & 3.4 & 4.4 & 8.6 & -0.2 & 0.2 \\
\hline $7580^{\mathrm{k}}$ & 37.38 & 33.19 & 10 & - & $12.0 \pm 4.0$ & $5.2 \pm 3.9$ & $-5.2 \pm 4.0$ & - & - & - & 3.1 & -3.7 \\
\hline $7585^{\mathrm{k}}$ & 39.80 & 34.81 & 10 & - & $17.7 \pm 4.3$ & $-3.2 \pm 4.3$ & $-3.3 \pm 4.3$ & - & - & - & 6.8 & -8.2 \\
\hline $7589^{k}$ & 39.89 & 32.76 & 10 & - & $8.0 \pm 2.0$ & $5.3 \pm 1.7$ & $21.1 \pm 2.0$ & - & - & - & -0.3 & 0.8 \\
\hline Antarctica (A & & & & & & & & & & & & \\
\hline CAS1 & -66.28 & 110.52 & 6.49 & 1839 & $-11.6 \pm 0.7$ & $3.7 \pm 0.6$ & $6.4 \pm 1.5$ & 5.2 & 5.5 & 12.4 & 0.2 & 0.2 \\
\hline DAV1 & -68.58 & 77.97 & 6.49 & 1849 & $-6.6 \pm 0.7$ & $-2.1 \pm 0.7$ & $4.0 \pm 1.5$ & 5.2 & 6.3 & 12.2 & 0.3 & 0.4 \\
\hline KERG & -49.35 & 70.26 & 6.13 & 1971 & $-5.3 \pm 0.7$ & $5.2 \pm 0.8$ & $6.5 \pm 1.5$ & 5.1 & 7.1 & 11.7 & 0.1 & 0.3 \\
\hline MAW1 & -67.60 & 62.87 & 7.00 & 1830 & $-3.6 \pm 0.7$ & $-3.5 \pm 0.7$ & $3.9 \pm 1.3$ & 5.8 & 7.2 & 10.2 & 0.2 & -0.1 \\
\hline MCM4 & -77.84 & 166.67 & 5.93 & 2060 & $-12.2 \pm 0.6$ & $10.6 \pm 0.7$ & $4.6 \pm 1.9$ & 4.5 & 5.7 & 15.5 & -0.9 & -0.5 \\
\hline SYOG & -69.01 & 39.58 & 4.00 & 1164 & $0.3 \pm 1.0$ & $-4.4 \pm 1.2$ & $5.8 \pm 2.2$ & 5.0 & 6.8 & 10.4 & -1.2 & 0.1 \\
\hline VESL & -71.67 & 357.16 & 2.39 & 648 & $8.2 \pm 1.8$ & $-4.7 \pm 1.4$ & $1.1 \pm 3.6$ & 5.0 & 4.8 & 9.8 & -1.7 & -3.3 \\
\hline ohig & -63.32 & 302.10 & 5.70 & 1330 & $10.1 \pm 0.9$ & $14.7 \pm 0.8$ & $8.7 \pm 1.8$ & 6.1 & 6.5 & 12.9 & -2.6 & 2.3 \\
\hline palm & -64.78 & 295.95 & 2.46 & 851 & $14.1 \pm 1.9$ & $11.7 \pm 1.5$ & $3.3 \pm 3.4$ & 5.7 & 5.3 & 9.5 & 1.8 & -1.5 \\
\hline Arabia (Ar) & & & & & & & & & & & & \\
\hline BAHR & 26.21 & 50.61 & 4.52 & 1592 & $26.9 \pm 0.6$ & $29.5 \pm 0.7$ & $-0.9 \pm 1.8$ & 3.0 & 4.9 & 9.1 & 0.2 & -0.6 \\
\hline KATZ & 33.00 & 35.69 & 4.18 & 590 & $19.3 \pm 0.7$ & $22.7 \pm 1.0$ & $3.9 \pm 2.4$ & 3.2 & 5.3 & 11.4 & -0.2 & 1.1 \\
\hline $7832^{\mathrm{k}}$ & 24.91 & 46.40 & 5 & - & $20.3 \pm 8.2$ & $25.9 \pm 8.5$ & $-4.3 \pm 8.4$ & - & - & - & -4.6 & -4.3 \\
\hline Australia $(\mathrm{Au})$ & & & & & & & & & & & & \\
\hline ALIC & -23.67 & 133.89 & 6.45 & 1574 & $57.0 \pm 0.4$ & $33.9 \pm 0.7$ & $2.0 \pm 1.5$ & 3.1 & 6.0 & 11.8 & 0.1 & -0.4 \\
\hline CEDU & -31.87 & 133.81 & 6.63 & 915 & $57.4 \pm 0.5$ & $31.5 \pm 0.6$ & $3.5 \pm 1.4$ & 3.4 & 5.2 & 10.0 & 0.5 & 0.5 \\
\hline DARW & -12.84 & 131.13 & 6.61 & 972 & $57.4 \pm 0.5$ & $38.4 \pm 1.0$ & $2.2 \pm 1.6$ & 3.4 & 8.2 & 12.2 & 0.2 & 0.2 \\
\hline HOB2 & -42.80 & 147.44 & 6.49 & 1873 & $54.2 \pm 0.5$ & $15.5 \pm 0.6$ & $2.5 \pm 1.4$ & 3.7 & 5.6 & 10.7 & 0.2 & -1.1 \\
\hline JAB1 & -12.66 & 132.89 & 3.40 & 501 & $58.2 \pm 0.8$ & $35.3 \pm 1.4$ & $-1.9 \pm 2.8$ & 3.1 & 6.1 & 10.5 & 1.2 & -2.6 \\
\hline KARR & -20.98 & 117.10 & 6.44 & 1576 & $56.7 \pm 0.4$ & $43.3 \pm 0.7$ & $1.0 \pm 1.4$ & 3.2 & 6.5 & 10.9 & 0.6 & 2.1 \\
\hline PERT & -31.80 & 115.89 & 7.36 & 2387 & $55.5 \pm 0.4$ & $42.1 \pm 0.6$ & $0.4 \pm 1.3$ & 3.3 & 6.3 & 11.8 & -0.4 & 1.8 \\
\hline TID2 & -35.40 & 148.98 & 5.00 & 1386 & $53.4 \pm 0.5$ & $19.5 \pm 0.7$ & $3.6 \pm 1.7$ & 3.2 & 4.9 & 10.0 & -0.1 & -1.4 \\
\hline TIDB & -35.40 & 148.98 & 8.00 & 2468 & $53.3 \pm 0.5$ & $19.4 \pm 0.6$ & $5.6 \pm 1.2$ & 4.3 & 6.7 & 12.0 & -0.2 & -1.5 \\
\hline TOW2 & -19.27 & 147.06 & 5.96 & 1500 & $53.5 \pm 0.5$ & $30.5 \pm 0.8$ & $3.5 \pm 1.6$ & 3.5 & 6.8 & 11.1 & -0.7 & -1.1 \\
\hline YAR1 & -29.05 & 115.35 & 8.00 & 2718 & $54.9 \pm 0.4$ & $41.9 \pm 0.6$ & $3.8 \pm 1.2$ & 3.5 & 6.3 & 11.7 & -0.9 & 0.8 \\
\hline auck & -36.60 & 174.83 & 5.29 & 1885 & $37.5 \pm 0.5$ & $5.1 \pm 0.7$ & $3.9 \pm 1.5$ & 3.4 & 5.7 & 8.9 & -1.8 & -2.3 \\
\hline $\operatorname{coco}^{1}$ & -12.19 & 96.83 & 4.55 & 1363 & $47.7 \pm 0.7$ & $43.0 \pm 1.3$ & $0.3 \pm 2.2$ & 3.9 & 8.5 & 12.4 & -1.1 & -2.2 \\
\hline dgar & -7.27 & 72.37 & 4.60 & 1556 & $29.8 \pm 0.7$ & $46.0 \pm 1.4$ & $2.5 \pm 2.2$ & 4.1 & 8.8 & 13.5 & -2.3 & 0.5 \\
\hline hyde & 17.42 & 78.55 & 5.47 & 268 & $32.2 \pm 0.8$ & $42.1 \pm 1.5$ & $2.1 \pm 2.5$ & 4.4 & 9.5 & 15.2 & -4.8 & 17.0 \\
\hline iisc & 13.02 & 77.57 & 5.98 & 1611 & $31.8 \pm 0.6$ & $42.4 \pm 1.0$ & $-0.1 \pm 1.7$ & 3.9 & 8.2 & 12.8 & -4.5 & 13.5 \\
\hline mald $^{1}$ & 4.19 & 73.53 & 1.39 & 485 & $30.1 \pm 2.8$ & $41.5 \pm 4.2$ & $8.6 \pm 6.5$ & 4.7 & 8.2 & 11.0 & -3.0 & 5.1 \\
\hline noum & -22.27 & 166.41 & 3.00 & 986 & $43.1 \pm 0.9$ & $21.8 \pm 1.6$ & $1.8 \pm 2.9$ & 3.1 & 6.7 & 10.2 & -1.9 & -1.8 \\
\hline Caribbean & & & & & & & & & & & & \\
\hline AVES & 15.67 & -63.62 & 3.87 & 29 & $11.5 \pm 0.9$ & $13.2 \pm 2.1$ & $5.0 \pm 3.4$ & 2.5 & 7.0 & 9.4 & 1.1 & 0.6 \\
\hline BARB & 13.09 & 300.39 & 3.09 & 569 & $12.6 \pm 1.0$ & $13.1 \pm 1.8$ & $-0.7 \pm 3.3$ & 3.3 & 7.3 & 11.8 & 0.2 & -0.3 \\
\hline CRO1 & 17.76 & 295.42 & 6.96 & 1828 & $10.7 \pm 0.4$ & $10.5 \pm 0.7$ & $2.5 \pm 1.5$ & 3.5 & 6.8 & 13.1 & 0.1 & -0.6 \\
\hline ROJO & 17.90 & -71.67 & 6.25 & 21 & $6.2 \pm 0.9$ & $11.5 \pm 1.9$ & $-5.5 \pm 3.7$ & 3.8 & 9.0 & 18.4 & 0.8 & -1.7 \\
\hline SANA & 12.52 & -81.73 & 6.25 & 29 & $4.8 \pm 0.9$ & $14.1 \pm 1.2$ & $0.8 \pm 3.8$ & 4.1 & 6.2 & 20.8 & 1.1 & 1.0 \\
\hline TDAD & 10.68 & -61.40 & 4.05 & 7 & $10.7 \pm 1.4$ & $15.9 \pm 3.2$ & $5.9 \pm 4.1$ & 3.2 & 8.1 & 9.4 & 1.5 & -1.1 \\
\hline isab & 18.47 & -67.05 & 4.18 & 26 & $9.3 \pm 1.4$ & $11.1 \pm 2.0$ & $-0.8 \pm 7.8$ & 4.2 & 7.0 & 30.8 & 0.4 & -0.4 \\
\hline pur3 & 18.46 & 292.93 & 3.56 & 1096 & $9.5 \pm 0.8$ & $7.5 \pm 1.4$ & $0.2 \pm 2.9$ & 3.2 & 7.0 & 13.7 & -0.2 & -3.2 \\
\hline Eurasia & & & & & & & & & & & & \\
\hline ARTU & 56.43 & 58.56 & 1.40 & 388 & $4.8 \pm 1.6$ & $24.5 \pm 1.6$ & $-0.9 \pm 5.6$ & 2.6 & 3.3 & 8.2 & -0.2 & -0.8 \\
\hline BOGO & 52.48 & 21.04 & 4.00 & 1293 & $12.7 \pm 0.5$ & $22.2 \pm 0.5$ & $0.9 \pm 2.0$ & 2.4 & 3.0 & 8.8 & 0.1 & 0.8 \\
\hline BOR1 & 52.28 & 17.07 & 6.26 & 2221 & $12.5 \pm 0.3$ & $20.4 \pm 0.4$ & $-1.9 \pm 1.2$ & 2.6 & 3.8 & 8.1 & -0.6 & -0.4 \\
\hline GLSV & 50.36 & 30.50 & 2.85 & 948 & $11.6 \pm 0.9$ & $22.4 \pm 0.8$ & $0.0 \pm 2.7$ & 2.9 & 3.5 & 8.1 & 0.6 & -1.0 \\
\hline GOPE & 49.91 & 14.79 & 5.30 & 1826 & $13.0 \pm 0.4$ & $21.6 \pm 0.5$ & $-4.9 \pm 1.7$ & 2.6 & 4.0 & 10.9 & -0.4 & 0.7 \\
\hline JOZE & 52.10 & 21.03 & 7.36 & 2577 & $12.5 \pm 0.3$ & $21.3 \pm 0.4$ & $-5.2 \pm 1.2$ & 3.0 & 4.1 & 10.8 & -0.1 & -0.2 \\
\hline KSTU & 55.99 & 92.79 & 2.92 & 713 & $-5.6 \pm 1.1$ & $25.3 \pm 1.3$ & $-0.4 \pm 2.9$ & 3.6 & 5.2 & 9.5 & -1.7 & -0.4 \\
\hline LAMA & 53.89 & 20.67 & 6.03 & 1844 & $12.8 \pm 0.4$ & $20.9 \pm 0.5$ & $-2.6 \pm 1.3$ & 3.1 & 4.4 & 8.2 & 0.2 & -0.1 \\
\hline
\end{tabular}


Table 1. (continued)

\begin{tabular}{|c|c|c|c|c|c|c|c|c|c|c|c|c|}
\hline \multirow[t]{2}{*}{ Site $^{a}$} & \multicolumn{2}{|c|}{ Position $^{\mathrm{b}}$} & \multirow{2}{*}{$\begin{array}{c}\Delta T^{\mathrm{c}} \\
\text { years }\end{array}$} & \multirow{2}{*}{$\begin{array}{l}\text { Total } \\
\text { Data }^{\mathrm{d}}\end{array}$} & \multicolumn{3}{|c|}{ Velocity } & \multicolumn{3}{|c|}{ WRMS $^{\mathrm{f}}$} & \multicolumn{2}{|c|}{ Rate Res. ${ }^{\mathrm{g}}$} \\
\hline & ${ }^{\circ} \mathrm{N}$ & ${ }^{\circ} \mathrm{E}$ & & & North & East & Vertical & $\mathrm{N}$ & E & $\mathrm{V}$ & $\mathrm{N}$ & E \\
\hline $\mathrm{MDVO}^{1}$ & 56.03 & 37.22 & 5.84 & 2030 & $10.5 \pm 0.5$ & $23.4 \pm 0.5$ & $-5.4 \pm 1.7$ & 3.7 & 4.3 & 12.8 & 0.7 & 0.3 \\
\hline NYAL & 78.93 & 11.87 & 7.97 & 2405 & $14.5 \pm 0.4$ & $10.4 \pm 0.4$ & $4.8 \pm 1.1$ & 3.5 & 4.1 & 10.0 & 0.8 & -0.3 \\
\hline POTS & 52.38 & 13.07 & 6.25 & 2131 & $13.4 \pm 0.3$ & $20.0 \pm 0.4$ & $-0.1 \pm 1.2$ & 2.4 & 3.5 & 7.7 & -0.2 & 0.0 \\
\hline TIXI & 71.63 & 128.87 & 2.23 & 764 & $-11.8 \pm 1.3$ & $16.2 \pm 1.2$ & $2.7 \pm 3.7$ & 3.6 & 3.9 & 9.4 & -0.1 & -0.5 \\
\hline WROC & 51.11 & 17.06 & 3.66 & 533 & $13.9 \pm 0.6$ & $20.6 \pm 0.6$ & $-0.7 \pm 2.1$ & 2.5 & 3.1 & 7.0 & 0.8 & -0.4 \\
\hline WTZR & 49.14 & 12.88 & 4.98 & 1770 & $13.5 \pm 0.4$ & $20.5 \pm 0.5$ & $-0.6 \pm 1.4$ & 2.4 & 3.4 & 7.2 & -0.1 & -0.3 \\
\hline ZWEN & 55.70 & 36.76 & 5.74 & 1841 & $9.6 \pm 0.5$ & $23.8 \pm 0.5$ & $-4.5 \pm 1.5$ & 3.1 & 4.4 & 9.5 & -0.3 & 0.7 \\
\hline bili & 68.08 & 166.44 & 1.33 & 369 & $-21.1 \pm 2.0$ & $11.4 \pm 1.6$ & $1.2 \pm 5.9$ & 3.1 & 3.1 & 8.1 & -6.1 & 1.9 \\
\hline brst & 48.38 & 355.50 & 2.16 & 528 & $15.8 \pm 0.9$ & $13.9 \pm 1.3$ & $-4.2 \pm 3.4$ & 2.2 & 4.0 & 7.0 & 0.9 & -3.8 \\
\hline brus & 50.80 & 4.36 & 6.69 & 2365 & $13.7 \pm 0.3$ & $18.0 \pm 0.4$ & $0.0 \pm 1.1$ & 2.5 & 3.8 & 7.5 & -0.7 & -0.8 \\
\hline casc & 38.69 & 350.58 & 3.67 & 980 & $15.2 \pm 0.6$ & $15.6 \pm 0.9$ & $0.6 \pm 2.1$ & 2.5 & 4.6 & 7.7 & 0.2 & -3.9 \\
\hline geno & 44.42 & 8.92 & 3.77 & 937 & $15.6 \pm 0.6$ & $19.8 \pm 0.7$ & $-4.3 \pm 2.3$ & 2.6 & 3.9 & 9.3 & 1.6 & -1.4 \\
\hline hers & 50.87 & 0.34 & 7.91 & 2417 & $15.0 \pm 0.4$ & $15.7 \pm 0.5$ & $0.1 \pm 1.1$ & 3.3 & 6.0 & 9.5 & 0.3 & -2.2 \\
\hline hofn & 64.27 & 344.80 & 3.24 & 1093 & $14.7 \pm 0.7$ & $11.4 \pm 0.8$ & $4.0 \pm 2.3$ & 2.7 & 3.8 & 7.7 & -0.3 & 1.5 \\
\hline irkt & 52.22 & 104.32 & 5.27 & 1867 & $-9.1 \pm 0.7$ & $26.5 \pm 0.7$ & $-1.8 \pm 1.7$ & 4.3 & 5.4 & 10.6 & -2.4 & 0.9 \\
\hline kiru & 67.86 & 20.97 & 7.48 & 2599 & $13.4 \pm 0.5$ & $15.9 \pm 0.6$ & $4.7 \pm 1.3$ & 4.2 & 5.9 & 12.5 & 0.8 & -0.9 \\
\hline kit3 & 39.13 & 66.89 & 6.24 & 1631 & $2.3 \pm 0.5$ & $28.6 \pm 0.7$ & $-2.6 \pm 1.4$ & 3.8 & 6.2 & 10.1 & -0.6 & 0.4 \\
\hline kosg & 52.18 & 5.81 & 8.00 & 2804 & $14.7 \pm 0.3$ & $17.7 \pm 0.3$ & $-1.1 \pm 0.9$ & 2.5 & 4.0 & 7.5 & 0.4 & -0.9 \\
\hline mans & 48.02 & 0.16 & 2.74 & 841 & $15.6 \pm 0.7$ & $16.7 \pm 1.0$ & $-2.7 \pm 2.5$ & 2.2 & 4.0 & 6.2 & 0.9 & -2.0 \\
\hline mets & 60.22 & 24.40 & 8.00 & 2684 & $11.4 \pm 0.3$ & $19.8 \pm 0.4$ & $2.7 \pm 1.0$ & 3.3 & 4.9 & 8.5 & -0.7 & -0.1 \\
\hline $\mathrm{mlvl}^{1}$ & 48.84 & 2.59 & 2.79 & 801 & $13.8 \pm 1.7$ & $16.8 \pm 1.1$ & $-8.2 \pm 2.8$ & 5.5 & 4.5 & 8.1 & -0.7 & -2.2 \\
\hline noto & 36.88 & 14.99 & 4.88 & 1657 & $16.4 \pm 0.5$ & $21.9 \pm 0.6$ & $0.2 \pm 1.7$ & 3.2 & 4.6 & 9.0 & 3.0 & -1.7 \\
\hline onsa & 57.40 & 11.93 & 8.00 & 2779 & $13.0 \pm 0.3$ & $15.9 \pm 0.4$ & $1.7 \pm 0.9$ & 2.7 & 4.2 & 7.5 & -0.7 & -2.4 \\
\hline penc & 47.79 & 19.28 & 4.67 & 1525 & $12.6 \pm 0.5$ & $22.6 \pm 0.5$ & $-7.3 \pm 2.1$ & 2.6 & 3.7 & 12.5 & -0.2 & 0.4 \\
\hline pol2 & 42.68 & 74.69 & 5.61 & 1632 & $2.1 \pm 0.5$ & $28.3 \pm 0.7$ & $0.2 \pm 1.4$ & 3.5 & 5.2 & 8.1 & 1.3 & 0.2 \\
\hline reyk & 64.14 & 338.04 & 4.95 & 1676 & $18.8 \pm 0.6$ & $-11.4 \pm 0.6$ & $-3.4 \pm 1.5$ & 3.4 & 4.4 & 7.9 & 4.0 & -19.8 \\
\hline sjdv & 45.88 & 4.68 & 3.20 & 1045 & $14.8 \pm 0.6$ & $18.6 \pm 0.7$ & $-0.6 \pm 2.3$ & 2.2 & 3.5 & 7.5 & 0.4 & -1.5 \\
\hline sofi & 42.56 & 23.39 & 3.48 & 972 & $10.4 \pm 0.7$ & $25.3 \pm 1.0$ & $-2.7 \pm 2.4$ & 2.9 & 4.7 & 9.3 & -1.8 & 1.4 \\
\hline sumk & 44.21 & 74.00 & 3.22 & 1002 & $-1.6 \pm 0.9$ & $27.8 \pm 0.9$ & $4.1 \pm 2.8$ & 3.3 & 4.4 & 10.7 & -2.6 & -0.1 \\
\hline toul & 43.56 & 1.48 & 3.84 & 1228 & $15.0 \pm 0.5$ & $18.5 \pm 0.7$ & $-2.3 \pm 1.9$ & 2.3 & 4.0 & 7.2 & 0.4 & -1.6 \\
\hline trom & 69.66 & 18.94 & 7.99 & 2139 & $15.5 \pm 0.4$ & $14.0 \pm 0.4$ & $1.7 \pm 1.0$ & 4.1 & 5.0 & 8.8 & 2.6 & -1.8 \\
\hline urum & 43.81 & 87.60 & 2.17 & 626 & $6.1 \pm 1.4$ & $30.4 \pm 1.6$ & $-6.9 \pm 3.9$ & 3.6 & 4.8 & 9.7 & 8.7 & 2.6 \\
\hline vill & 40.44 & 356.05 & 6.11 & 2100 & $14.2 \pm 0.4$ & $19.1 \pm 0.5$ & $-2.8 \pm 1.3$ & 2.8 & 4.4 & 8.3 & -0.7 & -0.9 \\
\hline wsrt & 52.91 & 6.60 & 3.51 & 1223 & $15.6 \pm 0.5$ & $17.3 \pm 0.6$ & $-1.5 \pm 1.9$ & 2.0 & 3.0 & 6.3 & 1.4 & -1.3 \\
\hline yakz & 62.03 & 129.68 & 3.13 & 945 & $-10.0 \pm 1.8$ & $22.8 \pm 1.0$ & $0.1 \pm 2.6$ & 6.8 & 4.5 & 9.2 & 1.8 & 3.2 \\
\hline zeck & 43.79 & 41.57 & 3.26 & 1112 & $9.4 \pm 0.6$ & $26.9 \pm 0.9$ & $3.1 \pm 2.3$ & 2.5 & 4.1 & 8.1 & 0.5 & 0.9 \\
\hline \multicolumn{13}{|l|}{ India (In) } \\
\hline HYDE & 17.42 & 78.55 & 5.47 & 268 & $32.2 \pm 0.8$ & $42.1 \pm 1.5$ & $2.1 \pm 2.5$ & 4.4 & 9.5 & 15.2 & 0.3 & 0.3 \\
\hline IISC & 13.02 & 77.57 & 5.98 & 1611 & $31.8 \pm 0.6$ & $42.4 \pm 1.0$ & $-0.1 \pm 1.7$ & 3.9 & 8.2 & 12.8 & -0.1 & -0.0 \\
\hline MALD $^{1}$ & 4.19 & 73.53 & 1.39 & 485 & $30.1 \pm 2.8$ & $41.5 \pm 4.2$ & $8.6 \pm 6.5$ & 4.7 & 8.2 & 11.0 & -1.8 & -1.6 \\
\hline $\operatorname{coco}^{1}$ & -12.19 & 96.83 & 4.55 & 1363 & $47.7 \pm 0.7$ & $43.0 \pm 1.3$ & $0.3 \pm 2.2$ & 3.9 & 8.5 & 12.4 & 17.9 & 3.0 \\
\hline dgar & -7.27 & 72.37 & 4.60 & 1556 & $29.8 \pm 0.7$ & $46.0 \pm 1.4$ & $2.5 \pm 2.2$ & 4.1 & 8.8 & 13.5 & -2.0 & 2.8 \\
\hline Nazca $(\mathrm{Nz})$ & & & & & & & & & & & & \\
\hline EISL & -27.15 & 250.62 & 6.94 & 1947 & $-9.0 \pm 0.7$ & $65.5 \pm 1.0$ & $1.8 \pm 1.7$ & 5.5 & 9.9 & 16.1 & -0.2 & -2.4 \\
\hline GALA & -0.74 & 269.70 & 4.91 & 1001 & $9.5 \pm 0.7$ & $53.0 \pm 1.1$ & $2.9 \pm 1.9$ & 4.0 & 7.4 & 10.6 & 1.3 & 1.9 \\
\hline GALD $^{\mathrm{m}}$ & -0.90 & -89.62 & 9 & - & $4.4 \pm 5.8$ & $56.3 \pm 8.2$ & $3.8 \pm 7.0$ & - & - & - & -4.4 & 5.1 \\
\hline EASA $^{\mathrm{m}}$ & -27.15 & -109.38 & 12 & - & $-9.3 \pm 0.7$ & $68.1 \pm 0.8$ & $3.9 \pm 0.8$ & - & - & - & -0.5 & 0.2 \\
\hline $7097^{\mathrm{k}}$ & -27.15 & -109.38 & 17 & - & $-9.3 \pm 0.8$ & $68.1 \pm 0.9$ & $3.9 \pm 0.9$ & - & - & - & -0.5 & 0.2 \\
\hline North Americ & & & & & & & & & & & & \\
\hline AMC2 & 38.80 & -104.52 & 2.20 & 760 & $-8.9 \pm 0.8$ & $-11.5 \pm 1.2$ & $-4.2 \pm 3.2$ & 2.2 & 4.0 & 6.8 & 0.6 & 1.7 \\
\hline AML5 & 35.15 & -101.88 & 4.93 & 1575 & $-8.1 \pm 0.5$ & $-12.3 \pm 0.9$ & $-5.4 \pm 2.2$ & 3.0 & 6.1 & 14.3 & 0.5 & 0.2 \\
\hline ANTO & 29.49 & -98.58 & 4.93 & 1661 & $-7.1 \pm 0.5$ & $-10.8 \pm 0.9$ & $-2.3 \pm 2.4$ & 3.2 & 6.5 & 17.0 & 0.3 & 0.2 \\
\hline AOML & 25.73 & -80.16 & 3.11 & 1051 & $-0.1 \pm 0.7$ & $-9.4 \pm 1.4$ & $-1.1 \pm 2.7$ & 2.5 & 6.2 & 9.7 & 0.3 & 1.0 \\
\hline ARL5 & 32.76 & -97.06 & 4.93 & 1585 & $-6.3 \pm 0.6$ & $-13.4 \pm 0.9$ & $-5.5 \pm 2.2$ & 3.7 & 6.1 & 14.6 & 0.5 & -1.3 \\
\hline ASHV & 35.60 & -82.55 & 3.16 & 1034 & $-0.6 \pm 0.7$ & $-13.9 \pm 1.1$ & $1.4 \pm 2.6$ & 2.7 & 5.1 & 9.1 & 0.7 & -0.3 \\
\hline ATL1 & 33.94 & -84.52 & 2.55 & 732 & $-2.1 \pm 0.9$ & $-13.9 \pm 1.6$ & $4.7 \pm 3.7$ & 2.8 & 5.8 & 11.5 & 0.0 & -0.9 \\
\hline BARH & 44.40 & -68.22 & 2.25 & 807 & $3.2 \pm 0.9$ & $-14.8 \pm 1.2$ & $0.4 \pm 3.3$ & 2.3 & 4.0 & 7.8 & -1.0 & 1.0 \\
\hline BRMU & 32.37 & -64.70 & 7.78 & 2697 & $6.1 \pm 0.3$ & $-12.4 \pm 0.5$ & $-0.4 \pm 1.1$ & 2.6 & 5.1 & 10.3 & 0.6 & -0.2 \\
\hline BRU1 & 43.89 & -69.95 & 4.97 & 1764 & $3.2 \pm 0.5$ & $-15.3 \pm 0.7$ & $0.2 \pm 1.8$ & 3.2 & 5.1 & 10.9 & -0.3 & 0.5 \\
\hline Ccv1 & 28.46 & -80.54 & 1.15 & 396 & $-3.6 \pm 1.9$ & $-15.9 \pm 3.3$ & $3.9 \pm 8.0$ & 2.5 & 5.3 & 11.1 & - & - \\
\hline Ccv3 & 28.46 & -80.55 & 2.42 & 836 & $-0.9 \pm 0.9$ & $-12.6 \pm 1.7$ & $0.5 \pm 3.6$ & 2.7 & 5.9 & 10.2 & - & - \\
\hline $\mathrm{CCV}^{* \mathrm{i}}$ & 28.46 & -80.55 & 3.56 & 1232 & $-1.2 \pm 0.6$ & $-13.2 \pm 1.1$ & $0.9 \pm 2.5$ & 2.7 & 5.8 & 10.6 & -0.6 & -1.9 \\
\hline CHL1 & 38.78 & -75.09 & 5.29 & 1806 & $0.7 \pm 0.4$ & $-15.4 \pm 0.7$ & $-0.7 \pm 1.8$ & 2.8 & 5.0 & 12.0 & -0.8 & -0.9 \\
\hline COLA & 34.08 & -81.12 & 2.00 & 712 & $-0.7 \pm 1.0$ & $-11.3 \pm 1.8$ & $-4.4 \pm 4.0$ & 2.4 & 5.3 & 8.8 & 0.1 & 1.8 \\
\hline COSA & 33.57 & -111.88 & 2.30 & 640 & $-12.4 \pm 1.3$ & $-12.1 \pm 1.6$ & $3.1 \pm 3.6$ & 3.5 & 5.2 & 8.9 & -0.4 & -1.1 \\
\hline DNRC & 39.16 & -75.52 & 2.77 & 856 & $0.7 \pm 0.8$ & $-13.9 \pm 1.3$ & $-3.0 \pm 3.1$ & 2.5 & 5.0 & 9.7 & -0.7 & 0.7 \\
\hline DQUA & 34.11 & -94.29 & 3.97 & 1364 & $-5.3 \pm 0.6$ & $-13.0 \pm 0.9$ & $7.5 \pm 2.5$ & 3.0 & 5.2 & 13.0 & 0.5 & -0.3 \\
\hline DUCK & 36.18 & -75.75 & 3.34 & 1054 & $1.3 \pm 0.7$ & $-14.4 \pm 1.0$ & $-8.7 \pm 2.8$ & 2.8 & 5.0 & 11.6 & 0.0 & -0.7 \\
\hline
\end{tabular}


Table 1. (continued)

\begin{tabular}{|c|c|c|c|c|c|c|c|c|c|c|c|c|}
\hline \multirow[t]{2}{*}{ Site $^{a}$} & \multicolumn{2}{|c|}{ Position $^{\text {b }}$} & \multirow{2}{*}{$\begin{array}{l}\Delta T_{,}^{\mathrm{c}} \\
\text { years }\end{array}$} & \multirow{2}{*}{$\begin{array}{l}\text { Total } \\
\text { Data }^{d}\end{array}$} & \multicolumn{3}{|c|}{ Velocity $^{\mathrm{e}}$} & \multicolumn{3}{|c|}{ WRMS $^{f}$} & Rate & es. ${ }^{g}$ \\
\hline & ${ }^{\circ} \mathrm{N}$ & ${ }^{\circ} \mathrm{E}$ & & & North & East & Vertical & $\mathrm{N}$ & $\mathrm{E}$ & $\mathrm{V}$ & $\mathrm{N}$ & $\mathrm{E}$ \\
\hline ERLA & 39.02 & -84.61 & 4.17 & 1263 & $-1.5 \pm 0.6$ & $-14.3 \pm 0.7$ & $0.4 \pm 1.8$ & 2.7 & 4.2 & 7.8 & 0.6 & 0.2 \\
\hline GAIT & 39.13 & -77.22 & 6.00 & 1705 & $0.6 \pm 0.3$ & $-14.0 \pm 0.5$ & $0.9 \pm 1.3$ & 2.3 & 4.5 & 8.6 & -0.1 & 0.6 \\
\hline GALB & 39.21 & -84.49 & 2.99 & 999 & $-1.2 \pm 0.7$ & $-14.2 \pm 1.1$ & $-1.5 \pm 2.7$ & 2.5 & 4.6 & 9.2 & 0.9 & 0.4 \\
\hline GDAC & 37.78 & -102.18 & 3.99 & 1407 & $-7.2 \pm 0.5$ & $-14.0 \pm 0.8$ & $2.4 \pm 2.0$ & 2.4 & 4.8 & 8.9 & 1.5 & -0.9 \\
\hline GLPT & 37.25 & -76.50 & 3.57 & 1227 & $1.3 \pm 0.6$ & $-14.4 \pm 1.0$ & $-3.9 \pm 2.3$ & 2.5 & 5.2 & 9.4 & 0.3 & -0.3 \\
\hline GODE & 39.02 & -76.83 & 7.64 & 2642 & $0.9 \pm 0.3$ & $-14.0 \pm 0.4$ & $-0.9 \pm 1.0$ & 2.6 & 4.5 & 8.9 & 0.0 & 0.6 \\
\hline HBRK & 38.30 & -97.29 & 5.71 & 1938 & $-7.1 \pm 0.4$ & $-13.7 \pm 0.6$ & $0.0 \pm 1.4$ & 3.0 & 4.7 & 9.1 & -0.2 & 0.0 \\
\hline HKLO & 35.68 & -95.86 & 5.71 & 1957 & $-6.3 \pm 0.4$ & $-13.4 \pm 0.5$ & $1.4 \pm 1.5$ & 3.0 & 4.5 & 9.7 & 0.1 & -0.3 \\
\hline HVLK & 37.65 & -99.11 & 4.59 & 1587 & $-7.6 \pm 0.5$ & $-13.8 \pm 0.7$ & $0.3 \pm 1.8$ & 2.7 & 4.6 & 9.3 & 0.0 & -0.4 \\
\hline KAN1 & 39.13 & -95.40 & 4.30 & 1510 & $-5.4 \pm 0.7$ & $-13.8 \pm 0.8$ & $2.2 \pm 2.0$ & 3.7 & 5.1 & 10.2 & 0.8 & 0.3 \\
\hline KELY & 66.99 & -50.94 & 5.33 & 1693 & $10.2 \pm 0.5$ & $-18.3 \pm 0.6$ & $-3.7 \pm 1.5$ & 3.1 & 4.4 & 8.6 & -0.2 & 0.0 \\
\hline KULU & 65.58 & -37.15 & 4.44 & 886 & $14.6 \pm 0.6$ & $-15.6 \pm 0.6$ & $0.6 \pm 2.1$ & 2.9 & 3.6 & 10.4 & -0.2 & -0.3 \\
\hline KYW1 & 24.58 & -81.65 & 3.56 & 999 & $-0.6 \pm 0.7$ & $-9.7 \pm 1.4$ & $2.6 \pm 2.6$ & 3.1 & 7.1 & 11.2 & 0.4 & 0.3 \\
\hline LMNO & 36.69 & -97.48 & 5.86 & 2014 & $-7.0 \pm 0.5$ & $-13.3 \pm 0.6$ & $-3.4 \pm 1.5$ & 3.2 & 5.0 & 10.1 & 0.0 & -0.1 \\
\hline LUBB & 33.54 & -101.84 & 4.93 & 1673 & $-9.0 \pm 0.6$ & $-12.2 \pm 1.0$ & $0.0 \pm 2.3$ & 3.6 & 6.9 & 16.4 & -0.4 & -0.2 \\
\hline MDO1 & 30.68 & -104.01 & 7.57 & 2626 & $-9.7 \pm 0.3$ & $-11.3 \pm 0.5$ & $0.8 \pm 1.0$ & 2.7 & 5.0 & 8.3 & -0.4 & -0.3 \\
\hline Mial & 25.73 & -80.16 & 2.82 & 921 & $-1.0 \pm 0.8$ & $-11.2 \pm 1.5$ & $3.1 \pm 3.4$ & 2.7 & 6.0 & 11.6 & - & - \\
\hline Mia3 & 25.73 & -80.16 & 2.83 & 824 & $-0.5 \pm 1.0$ & $-11.3 \pm 1.6$ & $5.3 \pm 3.2$ & 3.2 & 6.3 & 10.9 & - & - \\
\hline MIA*i & 25.73 & -80.16 & 5.29 & 1745 & $-0.8 \pm 0.5$ & $-11.6 \pm 0.8$ & $4.1 \pm 1.7$ & 3.0 & 6.0 & 11.3 & -0.4 & -1.2 \\
\hline MNP1 & 41.07 & -71.86 & 3.04 & 1031 & $3.5 \pm 0.9$ & $-15.9 \pm 1.1$ & $2.6 \pm 2.7$ & 3.2 & 5.0 & 9.4 & 0.7 & -0.8 \\
\hline NDSK & 37.38 & -95.64 & 4.00 & 1386 & $-5.9 \pm 0.6$ & $-13.6 \pm 0.8$ & $1.3 \pm 2.0$ & 2.9 & 4.5 & 9.3 & 0.4 & 0.0 \\
\hline NLIB & 41.77 & -91.57 & 7.83 & 2744 & $-4.8 \pm 0.3$ & $-14.1 \pm 0.4$ & $-1.5 \pm 1.0$ & 2.6 & 4.3 & 8.4 & 0.0 & 0.9 \\
\hline ODS5 & 31.87 & -102.32 & 4.93 & 1699 & $-9.5 \pm 0.6$ & $-11.8 \pm 1.0$ & $-1.7 \pm 2.3$ & 3.5 & 7.0 & 16.3 & -0.8 & -0.3 \\
\hline OMH1 & 41.78 & -95.91 & 2.23 & 768 & $-6.0 \pm 1.3$ & $-13.8 \pm 2.5$ & $6.9 \pm 4.2$ & 3.4 & 7.8 & 11.7 & 0.4 & 1.0 \\
\hline PASO & 31.77 & -106.41 & 4.89 & 1638 & $-10.8 \pm 0.5$ & $-12.0 \pm 0.8$ & $-5.7 \pm 2.0$ & 3.2 & 5.7 & 13.1 & -0.6 & -0.9 \\
\hline PATT & 31.78 & -95.72 & 3.61 & 1227 & $-5.6 \pm 0.6$ & $-12.9 \pm 1.2$ & $3.4 \pm 2.6$ & 2.7 & 6.1 & 11.8 & 0.7 & -1.0 \\
\hline PIT1 & 40.55 & -79.70 & 3.87 & 1286 & $-1.4 \pm 0.6$ & $-13.8 \pm 0.7$ & $-8.6 \pm 2.1$ & 2.8 & 4.2 & 9.2 & -1.2 & 1.2 \\
\hline PLTC & 40.18 & -104.73 & 5.86 & 2060 & $-10.4 \pm 0.4$ & $-13.4 \pm 0.5$ & $-2.3 \pm 1.3$ & 2.6 & 4.0 & 8.4 & -0.8 & 0.1 \\
\hline Por2 & 43.07 & -70.71 & 3.32 & 1112 & $2.5 \pm 0.8$ & $-16.1 \pm 1.0$ & $5.8 \pm 2.7$ & 3.1 & 5.0 & 10.7 & - & - \\
\hline Por4 & 43.07 & -70.71 & 1.55 & 517 & $2.1 \pm 1.8$ & $-12.3 \pm 2.7$ & $3.0 \pm 6.0$ & 3.4 & 6.0 & 11.6 & - & - \\
\hline $\mathrm{POR}^{* \mathrm{i}}$ & 43.07 & -70.71 & 5.29 & 1629 & $2.5 \pm 0.5$ & $-15.6 \pm 0.7$ & $5.5 \pm 1.7$ & 3.2 & 5.6 & 11.0 & -0.7 & 0.0 \\
\hline PRCO & 34.98 & -97.52 & 5.10 & 1722 & $-7.5 \pm 0.4$ & $-12.6 \pm 0.7$ & $-2.3 \pm 1.6$ & 2.7 & 4.9 & 9.7 & -0.5 & 0.1 \\
\hline PRDS & 50.87 & -114.29 & 3.33 & 1090 & $-13.4 \pm 0.6$ & $-14.0 \pm 0.8$ & $-2.0 \pm 2.1$ & 2.3 & 3.7 & 7.0 & -0.7 & 0.6 \\
\hline PSU1 & 40.81 & -77.85 & 3.16 & 935 & $0.4 \pm 0.7$ & $-14.9 \pm 1.0$ & $-1.8 \pm 2.8$ & 2.5 & 4.4 & 10.1 & -0.1 & 0.2 \\
\hline Rcm5 & 25.61 & -80.38 & 3.05 & 862 & $0.5 \pm 0.8$ & $-9.2 \pm 1.4$ & $-3.7 \pm 3.0$ & 2.8 & 5.8 & 10.8 & - & - \\
\hline Rcm6 & 25.61 & -80.38 & 1.89 & 623 & $-0.9 \pm 1.0$ & $-12.6 \pm 2.2$ & $6.4 \pm 4.5$ & 2.3 & 6.0 & 10.0 & - & - \\
\hline $\mathrm{RCM}^{* \mathrm{i}}$ & 25.61 & -80.38 & 4.94 & 1485 & $0.1 \pm 0.5$ & $-10.0 \pm 0.9$ & $-1.3 \pm 1.9$ & 2.6 & 6.1 & 11.5 & 0.6 & 0.4 \\
\hline RIC1 & 37.54 & -77.43 & 3.72 & 1144 & $0.8 \pm 0.6$ & $-14.0 \pm 0.8$ & $-1.7 \pm 2.3$ & 2.4 & 4.2 & 9.6 & 0.2 & 0.2 \\
\hline SAV1 & 32.14 & -81.70 & 2.09 & 643 & $-1.1 \pm 1.1$ & $-13.2 \pm 1.6$ & $1.9 \pm 4.1$ & 2.7 & 4.7 & 10.0 & -0.1 & -0.7 \\
\hline SOL1 & 38.32 & -76.45 & 5.27 & 1391 & $1.2 \pm 0.4$ & $-14.1 \pm 0.6$ & $-4.8 \pm 1.7$ & 2.4 & 4.8 & 10.4 & 0.2 & 0.3 \\
\hline STJO & 47.60 & -52.68 & 8.00 & 2813 & $10.1 \pm 0.3$ & $-15.7 \pm 0.4$ & $-1.3 \pm 1.0$ & 2.8 & 4.8 & 8.4 & 0.3 & -0.5 \\
\hline Stl3 & 38.61 & -89.76 & 3.93 & 1317 & $-4.1 \pm 0.6$ & $-13.7 \pm 0.9$ & $0.0 \pm 2.3$ & 2.6 & 5.2 & 11.2 & - & - \\
\hline St14 & 38.61 & -89.76 & 0.66 & 218 & $-11.9 \pm 4.6$ & $-14.9 \pm 7.4$ & $-24.0 \pm 13.1$ & 3.6 & 6.8 & 10.0 & - & - \\
\hline $\mathrm{STL}^{* \mathrm{i}}$ & 38.61 & -89.76 & 4.57 & 1535 & $-4.1 \pm 0.5$ & $-13.7 \pm 0.8$ & $0.0 \pm 2.0$ & 2.8 & 5.4 & 11.2 & 0.0 & 0.5 \\
\hline TCUN & 35.09 & -103.61 & 3.10 & 1017 & $-8.4 \pm 0.7$ & $-13.9 \pm 0.9$ & $5.0 \pm 2.5$ & 2.6 & 4.2 & 8.4 & 0.8 & -1.6 \\
\hline THU1 & 76.54 & -68.79 & 5.67 & 1803 & $3.5 \pm 0.4$ & $-22.0 \pm 0.4$ & $-0.7 \pm 1.4$ & 3.0 & 3.2 & 8.9 & -0.5 & -0.7 \\
\hline TMGO & 40.13 & -105.23 & 6.15 & 1882 & $-9.5 \pm 1.4$ & $-14.5 \pm 0.6$ & $-0.7 \pm 1.3$ & 10.6 & 5.1 & 8.1 & 0.3 & -1.0 \\
\hline USNA & 38.98 & -76.48 & 5.27 & 1377 & $-0.5 \pm 0.8$ & $-14.1 \pm 0.9$ & $0.2 \pm 1.5$ & 4.9 & 6.7 & 8.5 & -1.5 & 0.5 \\
\hline USNO & 38.92 & -77.07 & 3.67 & 1157 & $1.2 \pm 0.6$ & $-14.3 \pm 0.8$ & $-2.4 \pm 2.2$ & 2.4 & 4.4 & 8.7 & 0.4 & 0.3 \\
\hline VCIO & 36.07 & -99.22 & 5.71 & 1939 & $-6.3 \pm 0.5$ & $-12.6 \pm 0.6$ & $-0.6 \pm 1.6$ & 3.1 & 5.3 & 10.7 & 1.3 & 0.3 \\
\hline VIMS & 37.61 & -75.69 & 3.84 & 1207 & $1.4 \pm 0.5$ & $-14.5 \pm 0.9$ & $-2.3 \pm 2.1$ & 2.2 & 4.8 & 9.0 & 0.1 & -0.3 \\
\hline WES2 & 42.61 & -71.49 & 7.88 & 2721 & $2.3 \pm 0.3$ & $-14.6 \pm 0.4$ & $-2.3 \pm 1.0$ & 3.2 & 4.8 & 8.2 & -0.6 & 0.9 \\
\hline WHN1 & 42.74 & -103.33 & 2.17 & 759 & $-8.6 \pm 0.9$ & $-13.2 \pm 1.3$ & $4.7 \pm 3.5$ & 2.3 & 4.2 & 8.3 & 0.5 & 1.1 \\
\hline WLCI & 40.81 & -87.05 & 2.55 & 749 & $-4.3 \pm 0.9$ & $-13.7 \pm 1.5$ & $0.1 \pm 3.3$ & 2.6 & 5.3 & 9.6 & -1.2 & 1.3 \\
\hline WSMN & 32.41 & -106.35 & 5.68 & 1939 & $-10.0 \pm 0.5$ & $-12.1 \pm 0.6$ & $0.8 \pm 1.5$ & 3.4 & 5.3 & 9.9 & 0.2 & -0.8 \\
\hline algo & 45.96 & -78.07 & 8.00 & 2851 & $-0.6 \pm 0.3$ & $-16.3 \pm 0.3$ & $2.8 \pm 0.9$ & 2.7 & 3.9 & 7.5 & -1.0 & 0.2 \\
\hline chb1 & 45.65 & -84.47 & 5.29 & 1797 & $-1.3 \pm 0.5$ & $-14.7 \pm 0.6$ & $1.2 \pm 1.5$ & 2.9 & 4.7 & 8.8 & 0.8 & 1.6 \\
\hline chur & 58.76 & -94.09 & 7.68 & 2399 & $-6.8 \pm 0.4$ & $-18.2 \pm 0.4$ & $9.9 \pm 1.0$ & 3.2 & 4.3 & 8.1 & -1.1 & 0.5 \\
\hline $\operatorname{det} 1$ & 42.30 & -83.10 & 5.29 & 1847 & $-3.2 \pm 0.6$ & $-14.3 \pm 0.9$ & $0.5 \pm 1.7$ & 3.7 & 6.5 & 10.5 & -1.7 & 1.2 \\
\hline dubo & 50.26 & -95.87 & 4.20 & 1449 & $-7.6 \pm 0.5$ & $-17.8 \pm 0.6$ & $1.8 \pm 1.8$ & 2.3 & 3.8 & 7.7 & -1.2 & -1.0 \\
\hline flin & 54.73 & -101.98 & 4.56 & 1603 & $-10.5 \pm 0.5$ & $-17.5 \pm 0.6$ & $3.7 \pm 1.6$ & 2.8 & 4.0 & 7.9 & -1.9 & -0.4 \\
\hline kew1 & 47.23 & -88.62 & 3.56 & 1252 & $-5.1 \pm 0.7$ & $-17.6 \pm 0.9$ & $2.4 \pm 2.3$ & 2.9 & 4.8 & 9.2 & -1.4 & -1.0 \\
\hline mill & 43.00 & -87.89 & 3.56 & 1247 & $-3.0 \pm 0.7$ & $-17.3 \pm 0.9$ & $0.2 \pm 2.3$ & 2.8 & 4.6 & 9.4 & 0.5 & -1.8 \\
\hline neb3 & 46.32 & -84.15 & 3.66 & 1277 & $1.1 \pm 0.6$ & $-15.1 \pm 0.9$ & $4.4 \pm 2.2$ & 2.8 & 4.6 & 8.9 & 3.1 & 1.4 \\
\hline $\mathrm{nrc1}$ & 45.45 & -75.62 & 6.59 & 1939 & $0.7 \pm 0.3$ & $-15.8 \pm 0.4$ & $2.7 \pm 1.2$ & 2.4 & 4.2 & 8.3 & -0.6 & 0.5 \\
\hline oro_ & 44.90 & -68.67 & 2.33 & 615 & $4.2 \pm 0.9$ & $-16.3 \pm 1.2$ & $-0.9 \pm 3.4$ & 2.4 & 4.1 & 8.3 & 0.2 & -0.3 \\
\hline $\operatorname{sag} \overline{1}$ & 43.63 & -83.84 & 4.97 & 1715 & $-2.8 \pm 0.5$ & $-18.4 \pm 0.7$ & $1.2 \pm 1.7$ & 2.9 & 5.2 & 10.0 & -1.0 & -2.6 \\
\hline $\operatorname{sch} 2$ & 54.83 & -66.83 & 3.33 & 1150 & $5.2 \pm 0.7$ & $-17.8 \pm 0.8$ & $9.9 \pm 2.3$ & 2.8 & 4.1 & 8.5 & 0.5 & 0.4 \\
\hline stb1 & 44.80 & -87.31 & 3.56 & 1221 & $-2.5 \pm 0.8$ & $-16.8 \pm 1.0$ & $2.0 \pm 2.2$ & 3.2 & 5.0 & 8.4 & 0.7 & -0.8 \\
\hline stp1 & 44.30 & -91.90 & 3.56 & 1251 & $-4.4 \pm 0.6$ & $-16.0 \pm 1.0$ & $-2.2 \pm 2.4$ & 2.6 & 5.0 & 9.8 & 0.5 & -0.3 \\
\hline vcap & 44.26 & -72.58 & 4.57 & 1402 & $2.2 \pm 0.6$ & $-16.0 \pm 0.8$ & $1.4 \pm 2.0$ & 3.3 & 5.0 & 10.8 & -0.3 & -0.1 \\
\hline whp1 & 46.77 & -84.96 & 4.02 & 1335 & $-2.7 \pm 0.6$ & $-16.3 \pm 0.7$ & $3.2 \pm 2.1$ & 2.8 & 4.2 & 9.4 & -0.4 & 0.3 \\
\hline
\end{tabular}


Table 1. (continued)

\begin{tabular}{|c|c|c|c|c|c|c|c|c|c|c|c|c|}
\hline \multirow[t]{2}{*}{ Site $^{\mathrm{a}}$} & \multicolumn{2}{|c|}{ Position $^{\mathrm{b}}$} & \multirow{2}{*}{$\begin{array}{l}\Delta T T^{\mathrm{c}} \\
\text { years }\end{array}$} & \multirow{2}{*}{$\begin{array}{l}\text { Total } \\
\text { Data }^{d}\end{array}$} & \multicolumn{3}{|c|}{ Velocity $^{\mathrm{e}}$} & \multicolumn{3}{|c|}{$W_{R M S}^{f}$} & \multicolumn{2}{|c|}{ Rate Res. ${ }^{g}$} \\
\hline & ${ }^{\circ} \mathrm{N}$ & ${ }^{\circ} \mathrm{E}$ & & & North & East & Vertical & $\mathrm{N}$ & E & V & $\mathrm{N}$ & E \\
\hline wis1 & 46.71 & -92.02 & 3.56 & 1246 & $-4.9 \pm 0.6$ & $-17.5 \pm 0.8$ & $-1.3 \pm 2.3$ & 2.5 & 4.1 & 9.0 & 0.1 & -1 \\
\hline yell & 62.48 & -114.48 & 8.00 & 2840 & $-13.4 \pm 0.3$ & $-16.4 \pm 0.4$ & $2.6 \pm 1.0$ & 3.3 & 4.2 & 9.3 & -0.6 & \\
\hline you1 & 43.23 & -78.97 & 3.56 & 1254 & $-1.8 \pm 0.7$ & $-14.2 \pm 1.1$ & $0.9 \pm 2.6$ & 3.1 & 5.6 & 11.5 & -1.8 & \\
\hline bili & 68.08 & -193.56 & 1.33 & 369 & $-21.1 \pm 2.0$ & $11.4 \pm 1.6$ & $1.2 \pm 5.9$ & 3.1 & 3.1 & 8.1 & -1.0 & \\
\hline cha1 & 32.76 & -79.84 & 5.29 & 1824 & $-0.7 \pm 0.5$ & $-10.8 \pm 0.8$ & $2.2 \pm 1.7$ & 2.9 & 6.1 & 10.5 & -0.4 & \\
\hline drao & 49.32 & -119.62 & 8.00 & 2848 & $-13.5 \pm 0.3$ & $-12.4 \pm 0.4$ & $-0.4 \pm 1.0$ & 3.0 & 4.6 & 8.1 & 0.9 & \\
\hline fair & 64.98 & -147.50 & 8.00 & 2739 & $-22.7 \pm 0.4$ & $-7.4 \pm 0.4$ & $-2.3 \pm 1.2$ & 4.0 & 4.9 & 11.9 & -2.2 & \\
\hline hofn & 64.27 & -15.20 & 3.24 & 1093 & $14.7 \pm 0.7$ & $11.4 \pm 0.8$ & $4.0 \pm 2.3$ & 2.7 & 3.8 & 7.7 & -5.1 & 20.6 \\
\hline mem2 & 35.47 & -90.21 & 5.29 & 1779 & $-3.6 \pm 0.5$ & $-15.7 \pm 0.8$ & $0.8 \pm 1.7$ & 3.1 & 5.8 & 11.0 & 0.7 & -2 \\
\hline piel & 34.30 & -108.12 & 8.00 & 2826 & $-11.6 \pm 0.3$ & $-11.7 \pm 0.4$ & $1.6 \pm 0.9$ & 2.7 & 4.7 & 8.0 & -0.9 & -0.1 \\
\hline reyk & 64.14 & -21.96 & 4.95 & 1676 & $18.8 \pm 0.6$ & $-11.4 \pm 0.6$ & $-3.4 \pm 1.5$ & 3.4 & 4.4 & 7.9 & 0.2 & -0.2 \\
\hline \multicolumn{13}{|l|}{ Nubia $(\mathrm{Nu})$} \\
\hline GOUG & -40.35 & 350.12 & 2.42 & 653 & $15.3 \pm 2.0$ & $19.1 \pm 2.3$ & $-2.6 \pm 4.3$ & 5.7 & 7.6 & 13.1 & -0.9 & -1 \\
\hline Hart & -25.89 & 27.71 & 4.44 & 1427 & $18.3 \pm 1.5$ & $16.3 \pm 2.4$ & $-0.3 \pm 2.9$ & 8.0 & 14.5 & 18.8 & - & - \\
\hline Hark & -25.89 & 27.71 & 3.15 & 997 & $16.0 \pm 1.2$ & $17.9 \pm 1.6$ & $9.8 \pm 3.2$ & 4.4 & 7.0 & 12.9 & - & - \\
\hline Harb & -25.89 & 27.71 & 0.62 & 102 & $20.7 \pm 6.3$ & $11.3 \pm 7.7$ & $2.9 \pm 16.1$ & 4.3 & 6.1 & 11.0 & - & - \\
\hline $\mathrm{HAR}^{* \mathrm{i}}$ & -25.89 & 27.71 & 8.00 & 2528 & $17.4 \pm 0.7$ & $17.0 \pm 1.0$ & $3.7 \pm 1.5$ & 6.3 & 10.9 & 16.4 & 1.0 & -0.7 \\
\hline HRAO & -25.89 & 27.69 & 4.26 & 1099 & $15.5 \pm 0.8$ & $18.3 \pm 1.3$ & $0.8 \pm 2.2$ & 4.2 & 7.7 & 10.9 & -0.9 & \\
\hline Mas $1^{\mathrm{h}}$ & 27.76 & 344.37 & 6.58 & 2116 & $15.6 \pm 0.4$ & $16.2 \pm 0.6$ & $0.6 \pm 1.3$ & 2.8 & 5.8 & 10.0 & - & - \\
\hline Masp $^{\mathrm{h}}$ & 27.76 & 344.37 & 1.69 & 600 & $13.1 \pm 1.8$ & $14.6 \pm 3.0$ & $-8.1 \pm 5.2$ & 3.6 & 7.3 & 10.8 & - & - \\
\hline MAS $^{* \mathrm{n}}$ & 27.76 & 344.37 & 8.00 & 2443 & $15.5 \pm 0.3$ & $16.4 \pm 0.5$ & $1.0 \pm 1.1$ & 3.0 & 6.0 & 10.4 & 0.0 & \\
\hline SUTH & -32.38 & 20.81 & 2.68 & 891 & $15.9 \pm 1.4$ & $17.6 \pm 1.7$ & $-0.5 \pm 3.3$ & 4.4 & 6.6 & 10.8 & -1.0 & \\
\hline katz & 33.00 & 35.69 & 4.18 & 590 & $19.3 \pm 0.7$ & $22.7 \pm 1.0$ & $3.9 \pm 2.4$ & 3.2 & 5.3 & 11.4 & 3.8 & \\
\hline lamp & 35.50 & 12.61 & 1.77 & 586 & $17.5 \pm 1.3$ & $19.5 \pm 1.4$ & $4.5 \pm 4.4$ & 2.7 & 3.7 & 8.5 & 0.3 & 0.9 \\
\hline nico & 35.14 & 33.40 & 3.36 & 959 & $13.6 \pm 0.8$ & $19.0 \pm 0.9$ & $1.1 \pm 2.3$ & 3.0 & 4.2 & 8.0 & -2.2 & -3 \\
\hline $\mathrm{Iavh}^{\mathrm{h}}$ & 33.98 & 353.13 & 0.59 & 170 & $14.7 \pm 3.0$ & $24.0 \pm 5.1$ & $-4.3 \pm 12.1$ & 2.0 & 4.2 & 6.7 & - & - \\
\hline $\mathrm{Rabt}^{\mathrm{h}}$ & 33.98 & 353.14 & 0.56 & 133 & $18.7 \pm 7.4$ & $40.9 \pm 9.7$ & $-43.2 \pm 17.6$ & 4.8 & 7.2 & 11.6 & - & - \\
\hline $\mathrm{rab}^{* \mathrm{n}}$ & 33.98 & 353.14 & 2.54 & 303 & $18.9 \pm 1.1$ & $18.1 \pm 1.7$ & $-7.0 \pm 3.5$ & 3.0 & 5.5 & 8.7 & 2.4 & \\
\hline mali & -3.00 & 40.19 & 5.12 & 1714 & $12.7 \pm 0.7$ & $26.8 \pm 1.4$ & $1.5 \pm 2.3$ & 4.3 & 10.1 & 16.3 & -2.2 & 5 \\
\hline sey* & -4.67 & 55.48 & 3.98 & 276 & $8.0 \pm 1.3$ & $26.3 \pm 2.8$ & $1.9 \pm 3.7$ & 5.1 & 12.8 & 17.8 & -4.0 & 5.1 \\
\hline \multicolumn{13}{|l|}{ Okhotsk (Ok) } \\
\hline GS05 & 42.15 & 139.52 & 4.67 & 280 & $-12.3 \pm 0.8$ & $0.8 \pm 1.0$ & $-11.6 \pm 2.6$ & 3.7 & 5.6 & 12.9 & -0.9 & -1.4 \\
\hline STKW & 43.53 & 141.85 & 4.46 & 1257 & $-13.3 \pm 0.9$ & $3.7 \pm 1.0$ & $-2.0 \pm 3.1$ & 4.9 & 6.4 & 20.2 & -0.5 & 0.8 \\
\hline TKBA & 36.11 & 140.09 & 4.46 & 1031 & $-10.1 \pm 0.8$ & $-4.2 \pm 1.0$ & $-6.3 \pm 2.7$ & 4.1 & 6.0 & 16.0 & 1.6 & -1.4 \\
\hline TSKB & 36.11 & 140.09 & 7.00 & 2445 & $-12.7 \pm 0.7$ & $-4.2 \pm 0.7$ & $-2.3 \pm 1.3$ & 5.8 & 6.9 & 11.7 & -1.0 & -1.4 \\
\hline USUD & 36.13 & 138.36 & 8.00 & 2716 & $-10.1 \pm 0.6$ & $0.0 \pm 0.7$ & $0.2 \pm 1.3$ & 5.8 & 8.1 & 13.4 & 0.6 & 2.6 \\
\hline mag0 & 59.58 & 150.77 & 3.13 & 908 & $-23.8 \pm 1.1$ & $14.9 \pm 0.9$ & $-1.4 \pm 2.4$ & 4.2 & 4.3 & 7.6 & -5.7 & 1.3 \\
\hline petp & 53.07 & 158.61 & 2.23 & 694 & $-9.50 \pm 1.6$ & $0.2 \pm 1.3$ & $3.9 \pm 3.8$ & 3.4 & 4.2 & 9.5 & 12.9 & -6.1 \\
\hline
\end{tabular}

Pacific $(\mathrm{Pa})$

CHAT

FALE

HNLC

KOK1

KOKB

KWJ1

MARC

$\mathrm{Pama}^{\mathrm{h}}$

Tahi ${ }^{\mathrm{h}}$

Thti $^{\text {h }}$

THT*i

TRUK

Cice $^{\mathrm{h}}$

$\mathrm{Cic}^{\mathrm{h}}$

cic*i $^{\mathrm{i}}$

farb
guam

mkea

$\mathrm{pal}^{* \mathrm{i}, \mathrm{l}}$

scip

sni1

spmx

upo1

vndp

Philippine (Ph)

GSI

GSI3

OKTO

$\mathrm{Pala}^{\mathrm{h}}$
$-43.96$

$-13.83$

21.30

21.98

22.13
8.72

24.29

$-17.57$

$-17.58$

$-17.58$

$-17.58$

7.45

31.87

31.87

31.87

37.70

13.59
19.80

7.34

32.91

33.25

31.05

34.56

$183.43 \quad 5.24$

$188.00 \quad 2.00$

$202.14 \quad 2.00$

$200.24 \quad 3.56$

$200.34 \quad 7.99$

$\begin{array}{ll}167.73 & 4.79\end{array}$

$153.98 \quad 4.83$

$210.43 \quad 4.24$

$210.39 \quad 1.02$

$210.39 \quad 2.56$

$210.39 \quad 7.98$

$151.89 \quad 3.88$

$243.33 \quad 3.90$

$243.33 \quad 1.76$

$243.33 \quad 5.76$

$237.00 \quad 4.26$

$144.87 \quad 5.94$

$204.54 \quad 4.25$

$134.48 \quad 4.28$

$\begin{array}{ll}241.51 & 2.94\end{array}$

$240.48 \quad 5.11$

$\begin{array}{ll}244.53 & 2.19\end{array}$

$204.12 \quad 3.56$

$239.38 \quad 7.99$

$\begin{array}{rrr}1879 & 31.3 \pm 0.6 & -39.7 \pm 0.8 \\ 573 & 30.5 \pm 1.7 & -63.2 \pm 3.5 \\ 373 & 32.4 \pm 1.6 & -61.2 \pm 2.7 \\ 1235 & 32.0 \pm 0.8 & -61.8 \pm 1.4 \\ 2768 & 30.8 \pm 0.5 & -60.8 \pm 0.7 \\ 1442 & 25.6 \pm 0.8 & -67.9 \pm 1.2 \\ 1102 & 19.4 \pm 1.0 & -71.2 \pm 1.2 \\ 1423 & 31.5 \pm 1.4 & -63.1 \pm 3.1 \\ 254 & 33.6 \pm 3.7 & -62.1 \pm 6.8 \\ 618 & 31.2 \pm 1.5 & -63.3 \pm 2.7 \\ 2295 & 31.4 \pm 0.7 & -63.3 \pm 1.4 \\ 763 & 20.7 \pm 0.8 & -69.7 \pm 1.4 \\ 1316 & 15.5 \pm 0.6 & -39.1 \pm 0.9 \\ 613 & 20.5 \pm 1.2 & -39.2 \pm 1.8 \\ 1929 & 15.9 \pm 0.4 & -39.1 \pm 0.6 \\ 1364 & 22.3 \pm 0.5 & -39.6 \pm 0.7 \\ 2044 & 0.6 \pm 0.6 & -8.9 \pm 1.0 \\ 1394 & 31.2 \pm 0.7 & -61.9 \pm 1.0 \\ 563 & 19.0 \pm 0.8 & -64.2 \pm 1.8 \\ 723 & 20.4 \pm 0.7 & -42.1 \pm 1.1 \\ 1148 & 20.4 \pm 0.4 & -41.7 \pm 0.7 \\ 386 & 20.4 \pm 1.0 & -46.7 \pm 1.6 \\ 1242 & 34.6 \pm 1.2 & -62.9 \pm 1.7 \\ 2557 & 19.3 \pm 0.4 & -42.2 \pm 0.5\end{array}$

$3.6 \pm 1.5$
$-3.2 \pm 5.1$

$-1.1 \pm 5.2$

$2.6 \pm 2.8$

$1.6 \pm 1.3$

$0.0 \pm 2.3$

$5.6 \pm 2.4$

$1.7 \pm 3.5$

$1.2 \pm 11.3$

$8.9 \pm 4.5$

$3.6 \pm 1.7$

$5.8 \pm 2.8$

$-0.7 \pm 1.9$

$4.1 \pm 4.3$

$-0.3 \pm 1.3$

$0.0 \pm 1.9$

$5.2 \pm 1.8$

$1.1+2.1$

$-4.8 \pm 2.8$

$0.7 \pm 2.5$

$-0.3 \pm 1.6$

$2.4 \pm 3.6$

$-4.3 \pm 3.0$

$-1.0 \pm 1.1$

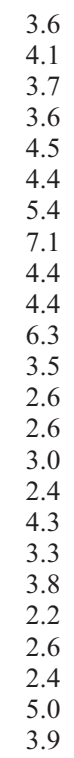

5.7

7.1

7.

7.7

8.1

7.8

18.2

9.2

9.4

14.8
7.3

4.9

4.5

4.8

4.5

8.7

6.3

9.6

4.6

4.7

4.8

8.5

5.7
13.0

12.3

13.4

13.3

14.6

15.2

23.5

15.1

15.2

20.0
13.5

13.5
7.9

7.9

8.2

9.2
14.9

10.5

14.4

6.7

8.4

7.6

14.4

10.5

$\begin{array}{rr}1.4 & 0.4 \\ -0.2 & -1.3 \\ 0.7 & 0.2 \\ 0.3 & -0.3 \\ -0.9 & 0.6 \\ -0.4 & -0.2 \\ -1.5 & -1.5 \\ - & - \\ - & - \\ - & - \\ -0.1 & 0.6 \\ 0.7 & -1.4 \\ - & - \\ - & - \\ -8.2 & 5.9 \\ -3.9 & 1.6 \\ -16.3 & 61.3 \\ -0.5 & -0.4 \\ 7.3 & 4.7 \\ -4.3 & 2.4 \\ -4.7 & 2.7 \\ -3.3 & -1.2 \\ 2.9 & -1.5 \\ -6.1 & 1.3\end{array}$

25.83

25.95

20.43
$131.23 \quad 4.67$

$131.29 \quad 3.58$

$\begin{array}{ll}136.08 & 3.91\end{array}$

$\begin{array}{ll}136.08 & 3.91 \\ 134.48 & 0.66\end{array}$
1147
104
17
178
$21.4 \pm 0.8 \quad-38.1 \pm 0.9$

$22.5 \pm 0.9 \quad-36.4 \pm 1.3$

$14.0 \pm 1.5 \quad-45.7 \pm 2.6$

$15.8 \pm 5.1 \quad-50.6 \pm 11.1$

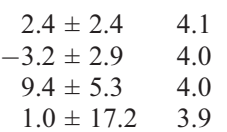

15.2

13.9

15.2

14.8

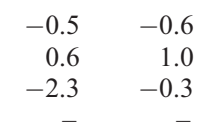


Table 1. (continued)

\begin{tabular}{|c|c|c|c|c|c|c|c|c|c|c|c|c|}
\hline \multirow[t]{2}{*}{ Site $^{a}$} & \multicolumn{2}{|c|}{ Position $^{\mathrm{b}}$} & \multirow{2}{*}{$\begin{array}{l}\Delta T T^{\mathrm{c}} \\
\text { years }\end{array}$} & \multirow{2}{*}{$\begin{array}{l}\text { Total } \\
\text { Data }^{\mathrm{d}}\end{array}$} & \multicolumn{3}{|c|}{ Velocity $^{\mathrm{e}}$} & \multicolumn{3}{|c|}{ WRMS $^{\mathrm{f}}$} & \multicolumn{2}{|c|}{ Rate Res. ${ }^{\mathrm{g}}$} \\
\hline & ${ }^{\circ} \mathrm{N}$ & ${ }^{\circ} \mathrm{E}$ & & & North & East & Vertical & $\mathrm{N}$ & E & $\mathrm{V}$ & $\mathrm{N}$ & $\mathrm{E}$ \\
\hline Pbls h,l & 7.34 & 134.48 & 2.53 & 385 & $19.3 \pm 1.3$ & $-65.0 \pm 2.9$ & $-7.1 \pm 8.8$ & 3.6 & 9.3 & 34.1 & - & - \\
\hline $\mathrm{PAL}^{* \mathrm{i}, 1}$ & 7.34 & 134.48 & 4.28 & 563 & $19.0 \pm 0.8$ & $-64.2 \pm 1.8$ & $-4.8 \pm 2.8$ & 3.8 & 9.6 & 14.4 & 0.8 & 0.2 \\
\hline ccjm & 27.10 & 142.18 & 4.29 & 1167 & $9.3 \pm 1.0$ & $-39.2 \pm 1.5$ & $-0.8 \pm 2.7$ & 5.2 & 8.7 & 15.8 & 0.3 & -5.0 \\
\hline gsi2 & 26.64 & 142.16 & 3.75 & 1058 & $6.6 \pm 1.2$ & $-33.1 \pm 1.7$ & $-2.8 \pm 3.1$ & 5.3 & 9.0 & 15.7 & -2.4 & 1.9 \\
\hline guam & 13.59 & 144.87 & 5.94 & 2044 & $0.6 \pm 0.6$ & $-8.9 \pm 1.0$ & $5.2 \pm 1.8$ & 4.3 & 8.7 & 14.9 & -5.1 & 46.3 \\
\hline haci & 33.07 & 139.82 & 4.43 & 1057 & $6.3 \pm 0.8$ & $-14.8 \pm 1.2$ & $-1.0 \pm 2.3$ & 4.2 & 7.0 & 12.3 & -5.5 & 9.6 \\
\hline oknw & 26.14 & 127.77 & 4.5 & 1278 & $-39.7 \pm 0.9$ & $35.3 \pm 1.2$ & $0.4 \pm 2.3$ & 5.0 & 7.7 & 13.1 & -65.6 & -73.0 \\
\hline s102 & 22.04 & 121.56 & 5.00 & 328 & $41.7 \pm 1.5$ & $-21.0 \pm 2.1$ & $24.8 \pm 3.4$ & 7.7 & 12.2 & 23.6 & 9.0 & 24.2 \\
\hline \multicolumn{13}{|c|}{ South America (Sa) } \\
\hline ASC1 & -7.95 & 345.59 & 4.66 & 1542 & $8.5 \pm 0.8$ & $-5.1 \pm 1.3$ & $0.3 \pm 2.2$ & 4.3 & 8.5 & 13.4 & -0.6 & 0.8 \\
\hline BOMJ & -13.26 & 316.58 & 2.92 & 661 & $10.1 \pm 1.2$ & $-5.7 \pm 2.1$ & $0.3 \pm 3.4$ & 4.0 & 8.3 & 11.8 & -0.6 & -0.6 \\
\hline BRAZ & -15.95 & 312.12 & 5.83 & 1434 & $10.5 \pm 0.6$ & $-5.5 \pm 1.0$ & $2.9 \pm 1.8$ & 4.0 & 7.6 & 13.1 & -0.2 & -0.7 \\
\hline FORT & -3.88 & 321.57 & 7.59 & 2634 & $11.5 \pm 0.5$ & $-6.9 \pm 0.8$ & $0.2 \pm 1.5$ & 4.4 & 8.7 & 16.1 & 0.9 & -1.7 \\
\hline IMPZ & -5.49 & 312.50 & 2.67 & 518 & $10.6 \pm 1.2$ & $-9.1 \pm 2.5$ & $-3.4 \pm 3.7$ & 3.7 & 8.9 & 11.7 & -0.1 & -4.0 \\
\hline KOUR & 5.25 & 307.19 & 7.99 & 2750 & $10.0 \pm 0.5$ & $-3.5 \pm 0.8$ & $1.7 \pm 1.4$ & 4.4 & 8.5 & 15.8 & -0.6 & 1.8 \\
\hline LPGS & -34.91 & 302.07 & 5.50 & 1607 & $10.8 \pm 0.8$ & $-1.6 \pm 1.0$ & $5.5 \pm 1.9$ & 5.3 & 8.0 & 14.2 & 0.4 & 1.3 \\
\hline MANU & -3.12 & 299.94 & 3.06 & 617 & $8.7 \pm 1.3$ & $-4.2 \pm 2.2$ & $-2.6 \pm 6.1$ & 4.6 & 9.0 & 29.2 & -1.6 & 0.8 \\
\hline PARA & -25.45 & 310.77 & 3.18 & 834 & $11.3 \pm 1.3$ & $-4.8 \pm 1.6$ & $7.0 \pm 3.1$ & 4.8 & 7.2 & 12.1 & 0.7 & -0.4 \\
\hline UEPP & -22.12 & 308.59 & 3.40 & 947 & $11.4 \pm 1.2$ & $-4.9 \pm 1.6$ & $6.0 \pm 3.0$ & 4.8 & 7.4 & 13.4 & 0.8 & -0.5 \\
\hline VICO & -20.76 & 317.13 & 2.80 & 737 & $10.2 \pm 1.3$ & $-6.5 \pm 1.9$ & $8.1 \pm 3.6$ & 4.3 & 7.3 & 12.7 & -0.5 & -1.5 \\
\hline \multicolumn{13}{|c|}{ South China (Cs) } \\
\hline S01R & 23.65 & 119.59 & 5.00 & 205 & $-17.8 \pm 0.9$ & $35.1 \pm 1.6$ & $9.1 \pm 3.4$ & 4.4 & 9.0 & 19.9 & -1.3 & 1.1 \\
\hline SHAO & 31.10 & 121.20 & 5.96 & 1827 & $-16.7 \pm 0.5$ & $34.0 \pm 0.8$ & $-0.1 \pm 1.5$ & 3.6 & 6.7 & 10.1 & 0.2 & 0.4 \\
\hline WUHN & 30.53 & 114.36 & 4.93 & 1642 & $-14.8 \pm 0.6$ & $33.9 \pm 0.8$ & $0.2 \pm 1.8$ & 3.8 & 5.9 & 10.8 & 0.3 & -0.7 \\
\hline bjfs & 39.61 & 115.89 & 1.20 & 419 & $-13.8 \pm 2.4$ & $29.9 \pm 2.8$ & $-3.9 \pm 7.5$ & 3.4 & 4.9 & 11.0 & 1.7 & -3.7 \\
\hline kunm & 25.03 & 102.80 & 2.23 & 684 & $-21.2 \pm 1.4$ & $32.3 \pm 2.5$ & $-4.3 \pm 4.5$ & 3.7 & 7.8 & 13.1 & -9.6 & -3.5 \\
\hline oknw & 26.14 & 127.77 & 4.5 & 1278 & $-39.7 \pm 0.9$ & $35.3 \pm 1.2$ & $0.4 \pm 2.3$ & 5.0 & 7.7 & 13.1 & -21.3 & 2.4 \\
\hline urum & 43.81 & 87.60 & 2.17 & 626 & $6.1 \pm 1.4$ & $30.4 \pm 1.6$ & $-6.9 \pm 3.9$ & 3.6 & 4.8 & 9.7 & 12.4 & -6.4 \\
\hline xian & 34.37 & 109.22 & 3.48 & 991 & $-14.7 \pm 0.9$ & $34.5 \pm 1.1$ & $0.1 \pm 2.5$ & 3.8 & 5.2 & 10.0 & -1.1 & -0.6 \\
\hline \multicolumn{13}{|l|}{ Sierra (Sr) } \\
\hline CMBB & 38.03 & 239.61 & 7.06 & 2133 & $-7.1 \pm 0.4$ & $-20.1 \pm 0.6$ & $3.2 \pm 1.9$ & 3.7 & 6.2 & 19.4 & -0.1 & 2.0 \\
\hline MUSB & 37.17 & 240.69 & 3.15 & 804 & $-6.8 \pm 0.7$ & $-24.2 \pm 1.1$ & $3.6 \pm 2.6$ & 2.4 & 4.7 & 8.5 & -0.7 & -2.7 \\
\hline ORVB & 39.55 & 238.50 & 4.00 & 1225 & $-8.5 \pm 0.5$ & $-23.7 \pm 0.7$ & $-1.8 \pm 2.1$ & 2.2 & 4.2 & 9.5 & -0.5 & -0.5 \\
\hline SUTB & 39.21 & 238.18 & 3.70 & 1061 & $-8.1 \pm 0.6$ & $-23.0 \pm 0.8$ & $-2.7 \pm 2.1$ & 2.4 & 4.1 & 7.7 & 0.1 & -0.1 \\
\hline UCD1 & 38.54 & 238.25 & 3.82 & 896 & $-7.3 \pm 0.6$ & $-23.5 \pm 0.8$ & $-8.8 \pm 2.4$ & 2.6 & 4.2 & 10.3 & 0.9 & -1.1 \\
\hline CEDA & 35.75 & -118.59 & 5.12 & 26 & $-5.1 \pm 1.0$ & $-21.0 \pm 1.5$ & $-2.7 \pm 2.4$ & 3.6 & 6.2 & 7.7 & -0.5 & 0.4 \\
\hline KMED & 36.02 & -118.14 & 6.90 & 29 & $-4.5 \pm 0.7$ & $-20.2 \pm 0.8$ & $-1.3 \pm 2.0$ & 3.2 & 4.8 & 9.0 & 0.5 & 0.7 \\
\hline SPRN & 36.18 & -118.73 & 5.08 & 27 & $-7.9 \pm 1.1$ & $-18.0 \pm 1.6$ & $-4.5 \pm 3.3$ & 3.9 & 6.9 & 13.1 & -3.2 & -0.8 \\
\hline TIOG & 37.93 & -119.25 & 4.00 & 11 & $-6.4 \pm 0.9$ & $-24.0 \pm 2.1$ & $-1.1 \pm 3.5$ & 2.2 & 6.1 & 8.1 & 1.3 & 0.3 \\
\hline quin & 39.97 & 239.06 & 7.99 & 2371 & $-8.0 \pm 0.4$ & $-19.8 \pm 0.5$ & $-2.6 \pm 1.2$ & 3.7 & 5.5 & 11.2 & -0.5 & 3.7 \\
\hline \multicolumn{13}{|l|}{ Somalia (So) } \\
\hline MALI & -3.00 & 40.19 & 5.12 & 1714 & $12.7 \pm 0.7$ & $26.8 \pm 1.4$ & $1.5 \pm 2.3$ & 4.3 & 10.1 & 16.3 & 0.0 & -0.1 \\
\hline Sey $1^{\mathrm{h}}$ & -4.67 & 55.48 & 5.63 & 360 & $9.9 \pm 1.0$ & $33.0 \pm 2.4$ & $10.6 \pm 3.1$ & 5.9 & 15.6 & 22.2 & - & - \\
\hline SEY*o & -4.67 & 55.48 & 3.98 & 276 & $8.0 \pm 1.3$ & $26.3 \pm 2.8$ & $1.9 \pm 3.7$ & 5.1 & 12.8 & 17.8 & 0.0 & 0.2 \\
\hline \multicolumn{13}{|l|}{ Sunda $(\mathrm{Su})$} \\
\hline BAKO & -6.49 & 106.85 & 2.83 & 765 & $-8.5 \pm 1.3$ & $24.0 \pm 2.1$ & $-4.6 \pm 3.9$ & 4.2 & 8.3 & 14.6 & -0.4 & 0.5 \\
\hline NTUS & 1.35 & 103.68 & 3.27 & 841 & $-6.0 \pm 1.1$ & $27.8 \pm 2.1$ & $-1.8 \pm 3.2$ & 4.1 & 9.2 & 13.5 & 0.3 & -0.4 \\
\hline kunm & 25.03 & 102.80 & 2.23 & 684 & $-21.2 \pm 1.4$ & $32.3 \pm 2.5$ & $-4.3 \pm 4.5$ & 3.7 & 7.8 & 13.1 & -6.7 & 4.8 \\
\hline
\end{tabular}

${ }^{a}$ Upper case sites are in stable plate interior used to calculate angular velocity for that plate; lowercase sites are in boundary zone or other deforming zone, sites with asterisk indicate composite site (see text).

${ }^{\mathrm{b}}$ Site location in decimal degrees.

${ }^{\mathrm{c}}$ Length of time series.

${ }^{\mathrm{d}}$ Number of station days.

${ }^{\mathrm{e}}$ Velocities in $\mathrm{mm} / \mathrm{yr}$ relative to ITRF-97, \pm 1 standard error

${ }^{\mathrm{f}}$ Weighted root mean square scatter of the daily position estimates about a best fit straight line.

${ }^{\mathrm{g}}$ Residual rate with respect to stable plate angular velocity.

${ }^{\mathrm{h}}$ Site that is part of a composite site solution (see footnotes $\mathrm{i}$ and $\mathrm{n}$ ).

${ }^{i}$ Composite site solution that is made up of two sites linked with an offset (see text).

${ }^{\mathrm{j}}$ Site Ankr without data after 15 August 1999 to avoid coseismic and postseismic effects of $M>7$ earthquakes.

${ }^{\mathrm{k}}$ SLR site.

${ }^{1}$ Site for which an offset has been calculated even though no change in antenna type, height, or dome change was reported.

${ }^{\mathrm{m}}$ DORIS site (GALD is really GALB).

${ }^{\mathrm{n}} \mathrm{A}$ published site tie was used to link two sites together.

${ }^{\circ}$ Site Sey1 without 1995 and 1996 data (see text). 

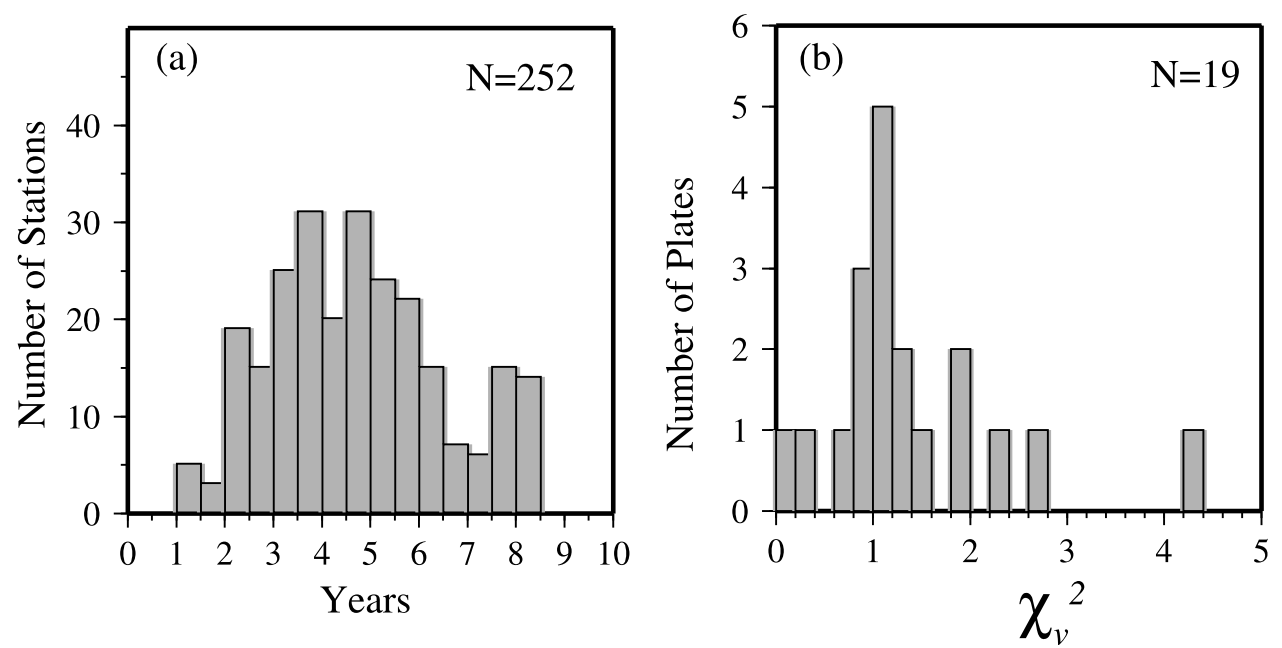

Figure 2. (a) Histogram showing length of time series for the sites analyzed in this study. (b) Histogram of $\chi_{v}^{2}\left(\chi^{2}\right.$ per degree of freedom) calculated for the 19 plates and blocks described in this study.

[9] The total time span of observations has an important influence on the uncertainty of site velocities from space geodetic data [Coates et al., 1985; Dixon, 1991; Zhang et al., 1997; Mao et al., 1999]. Table 1 and Figure 2a show the time span of data for the sites used in this study. The great majority of sites in this study have recorded more than 300 days per year for more than 3.0 years, the mean being 4.7 years. Figure 3 shows the expected evolution of velocity error as a function of time for a typical station that operates essentially continuously, recording 300 days per year. By reference to Figure 3, we can see that on average, the velocity errors for the horizontal components of a 4-year time series will lie in the range from 0.5 to $1.5 \mathrm{~mm} / \mathrm{yr}$. However, in order to obtain adequate geographic coverage we have also used stations with time spans as short as 1.4 years. This type of station will have horizontal component velocity uncertainties of $\sim 2-5 \mathrm{~mm} / \mathrm{yr}$ with the analytical techniques used here.

[10] Table 1 lists the available data per plate. Eight plates or blocks (Amuria, Anatolia, Arabia, India, Philippine, Somalia, South China, and Sunda) have less than 10 velocity data (i.e., less than five sites, with two horizontal velocity components per site); hence their angular velocities may not be well determined.

[11] From the site velocity data listed in Table 1 we seek the best fit angular velocity vector (Euler vector) describing the relative motion of adjacent plates (the relative velocities of nonadjacent plates can also be derived, but the results are more difficult to test against geologic data). We first select sites thought to be representative of stable plate interiors, using standard geologic and seismological criteria. For example, for the Eurasian

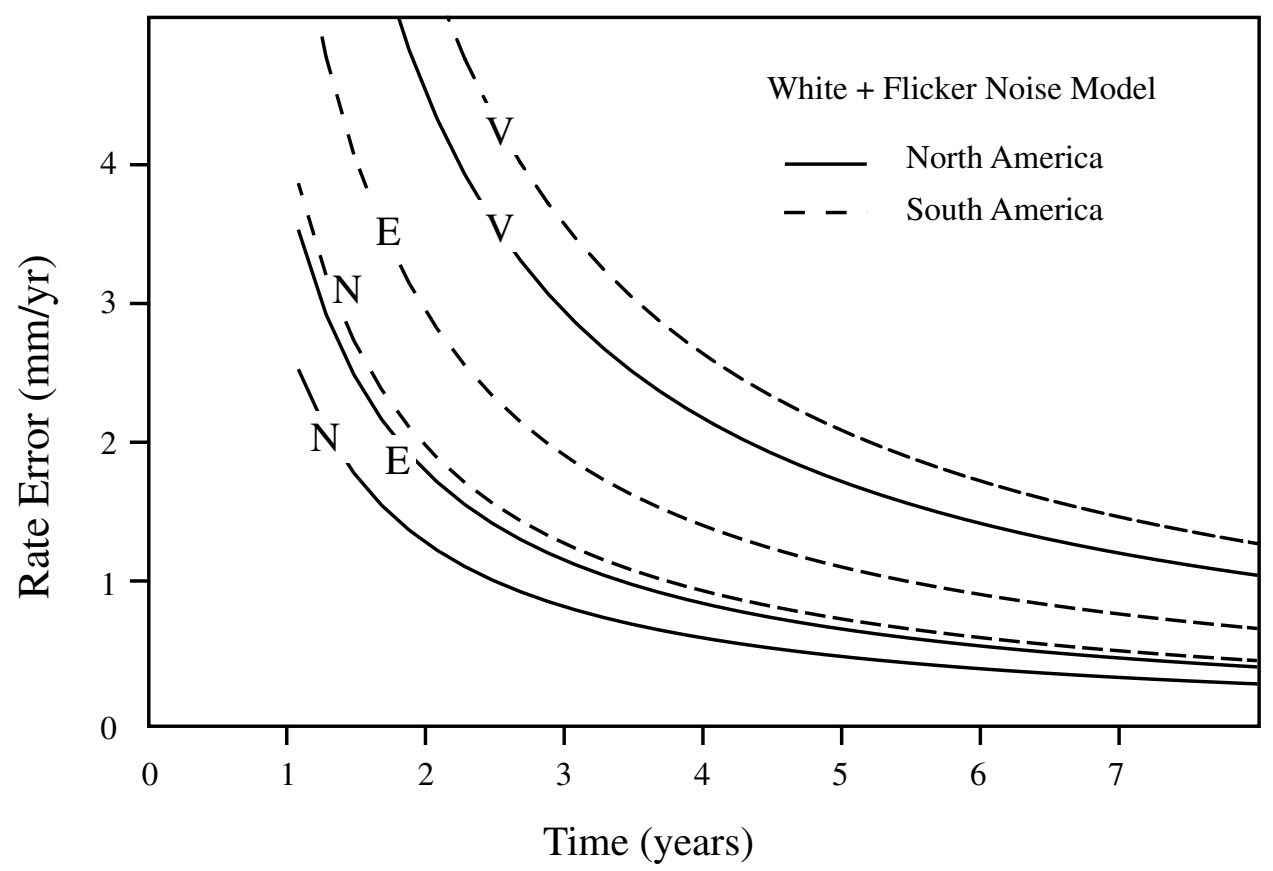

Figure 3. Estimate of GPS velocity error as a function of time this study, calculated according to the model of Mao et al. [1999] as modified by Dixon et al. [2000a]. Solid lines show north (N), east (E) and vertical (V) error for a typical North American site, assuming WRMS for each component is 3, 5, and $10 \mathrm{~mm}$, close to the mean of the 64 sites listed in Table 1. Dashed lines show corresponding curves based on mean South American site. 
Table 2. Plate Angular Velocity Relative to ITRF-97 in Geographic Coordinates

\begin{tabular}{|c|c|c|c|c|c|c|c|c|c|c|c|c|}
\hline \multirow[t]{2}{*}{ Plate $^{\mathrm{a}}$} & \multirow[t]{2}{*}{$\lambda,{ }^{\mathrm{b}}{ }^{\circ} \mathrm{N}$} & \multirow[t]{2}{*}{$\phi,{ }^{\mathrm{b}} \mathrm{E}$} & \multirow{2}{*}{$\begin{array}{c}\omega,{ }^{\mathrm{b}} \mathrm{deg} / \\
\mathrm{Myr}\end{array}$} & \multirow[t]{2}{*}{$\sigma_{\mathrm{maj}}^{\mathrm{c}}$} & \multirow[t]{2}{*}{$\sigma_{\min }{ }^{\mathrm{c}}$} & \multirow[t]{2}{*}{$\zeta,{ }^{\mathrm{d}}$ deg } & \multirow{2}{*}{$\begin{array}{c}\sigma_{\omega}, \\
\operatorname{deg} / \mathrm{Myr}\end{array}$} & \multirow{2}{*}{$\begin{array}{c}\text { Number } \\
\text { of } \\
\text { Sites }\end{array}$} & \multirow[t]{2}{*}{$\chi_{\nu}^{2 \mathrm{f}}$} & \multicolumn{2}{|c|}{ RMS Res. $^{\mathrm{g}}$} & \multirow{2}{*}{$\begin{array}{c}\text { Mean } \\
\text { Rate } \\
\text { Res. }\end{array}$} \\
\hline & & & & & & & & & & $\mathrm{N}$ & $\mathrm{E}$ & \\
\hline Am & 63.75 & -133.76 & 0.327 & 23.5 & 1.6 & -64 & 0.057 & 3 & 2.30 & 1.2 & 0.2 & 1.1 \\
\hline An & 58.48 & -134.00 & 0.226 & 1.6 & 1.0 & 32 & 0.010 & 7 & 1.02 & 0.9 & 1.3 & 1.0 \\
\hline $\mathrm{Ar}$ & 51.47 & 2.89 & 0.521 & 3.1 & 0.7 & -70 & 0.024 & 3 & 0.85 & 2.6 & 2.6 & 2.6 \\
\hline At & 42.19 & 26.18 & 0.882 & 8.2 & 0.7 & -65 & 0.889 & 4 & 1.60 & 3.7 & 4.5 & 4.1 \\
\hline $\mathrm{Au}$ & 34.86 & 38.26 & 0.627 & 1.2 & 0.4 & -65 & 0.004 & 11 & 2.78 & 0.6 & 1.4 & 1.3 \\
\hline $\mathrm{Ca}$ & 37.74 & -90.86 & 0.272 & 3.9 & 1.1 & -50 & 0.021 & 6 & 0.91 & 0.9 & 1.0 & 1.2 \\
\hline Cs & 54.58 & -109.21 & 0.340 & 16.6 & 1.0 & -40 & 0.007 & 3 & 1.27 & 0.8 & 0.8 & 1.0 \\
\hline $\mathrm{Eu}$ & 58.27 & -102.21 & 0.257 & 1.5 & 0.4 & 34 & 0.003 & 15 & 1.02 & 0.6 & 0.5 & 0.7 \\
\hline In & 53.65 & -13.99 & 0.483 & 11.7 & 0.5 & 80 & 0.013 & 3 & 0.26 & 1.0 & 1.0 & 1.0 \\
\hline $\mathrm{Na}$ & -2.39 & -79.08 & 0.199 & 0.8 & 0.3 & -6 & 0.002 & 64 & 1.05 & 0.6 & 0.8 & 0.9 \\
\hline $\mathrm{Nu}$ & 52.25 & -80.18 & 0.253 & 1.6 & 0.9 & -83 & 0.004 & 5 & 0.82 & 0.8 & 0.9 & 1.1 \\
\hline $\mathrm{Nz}$ & 44.45 & -99.49 & 0.647 & 2.9 & 0.6 & 5 & 0.011 & 5 & 1.96 & 2.1 & 2.7 & 2.5 \\
\hline Ok & -37.92 & -58.14 & 0.428 & 6.1 & 1.4 & -85 & 0.124 & 5 & 4.36 & 1.0 & 1.6 & 1.8 \\
\hline $\mathrm{Pa}$ & -64.21 & 112.74 & 0.655 & 0.7 & 0.4 & 75 & 0.004 & 9 & 1.20 & 0.8 & 0.9 & 1.1 \\
\hline $\mathrm{Ph}$ & -46.52 & -30.41 & 0.910 & 2.2 & 0.4 & -35 & 0.046 & 4 & 1.03 & 1.3 & 0.6 & 1.3 \\
\hline $\mathrm{Sa}$ & -25.83 & -135.38 & 0.106 & 7.7 & 2.8 & -80 & 0.003 & 11 & 1.31 & 0.7 & 1.6 & 1.5 \\
\hline So & 53.51 & -101.55 & 0.310 & 0.9 & 0.1 & 42 & 0.002 & 2 & 0.01 & 0.0 & 0.1 & 0.1 \\
\hline $\mathrm{Sr}$ & 10.87 & -112.00 & 0.445 & 9.8 & 0.5 & -14 & 0.099 & 9 & 1.96 & 1.2 & 1.2 & 1.5 \\
\hline$\underline{\mathrm{Su}}$ & 38.86 & -86.94 & 0.393 & 10.2 & 0.8 & -20 & 0.062 & 2 & 0.24 & 0.3 & 0.4 & 0.5 \\
\hline
\end{tabular}

${ }^{\text {a }}$ Plate abbreviations are listed in Table 1.

${ }^{\mathrm{b}}$ Parameter $\lambda$ is latitude and $\phi$ is longitude of the rotation pole in decimal degrees; $\omega$ is the rotation rate.

${ }^{\mathrm{c}}$ Two-dimensional 1-sigma lengths in degrees of the semi-major $\sigma_{\text {maj }}$ and semi-minor axes $\sigma_{\min }$ of the pole error ellipse.

${ }^{\mathrm{d}}$ The parameter $\zeta$ is the azimuth of the semi-major ellipse axis in degrees clockwise from north.

${ }^{\mathrm{e}}$ List of sites used is given in Table 1.

${ }^{\mathrm{f}}$ The parameter $\chi^{2}$ per degree of freedom.

${ }^{\mathrm{g}}$ Root mean square of the rate residual north and east components in $\mathrm{mm} / \mathrm{yr}$.

${ }^{\mathrm{h}}$ Mean rate residual in $\mathrm{mm} / \mathrm{yr}$.

plate we omit all data likely to be influenced by deformation associated with the northward motion of Africa, Arabia, or India, as indicated by seismicity, and also all sites east of longitude $130^{\circ} \mathrm{E}$ because they may lie within the deforming zone between Eurasia and North America or lie on the North American plate. In most cases we also choose sites located $>100 \mathrm{~km}$ from significant plate boundary zone-related seismicity. In this way we hope to avoid seismic cycle effects, including interseismic strain accumulation, which can extend far from faults, as well as postseismic effects, which can persist long after an earthquake because of viscous relaxation in the lower crust and upper mantle [e.g., Pollitz, 1992; Pollitz and Sacks, 1994]. By avoiding these effects our measured decadal velocities should be similar to longer-term plate velocities. In cases where we have had to violate this criterion in order to obtain sufficient data we have tested sensitivity to first-order strain effects via simple elastic half-space models.

[12] Next we invert the site velocity data to derive best fit angular velocities for each plate, termed here ITRF-97 angular velocities (Tables 2 and 3), minimizing the weighted, least squares

Table 3. Plate Angular Velocities Relative to ITRF-97 in Cartesian Coordinates With Covariance Matrix

\begin{tabular}{|c|c|c|c|c|c|c|c|c|c|}
\hline Plate $^{\mathrm{a}}$ & $\omega X^{b}$ & $\omega Y^{b}$ & $\omega Z^{b}$ & $\mathrm{xx}^{\mathrm{c}}$ & $x y^{c}$ & $\mathrm{xz}^{\mathrm{c}}$ & $\mathrm{yy}^{\mathrm{c}}$ & $\mathrm{yz}^{\mathrm{c}}$ & $\mathrm{zz}^{\mathrm{c}}$ \\
\hline Am & -1.746969 & -1.824311 & 5.121903 & 0.857324 & -1.063607 & -1.096796 & 1.347375 & 1.378061 & 1.438827 \\
\hline An & -1.431031 & -1.481635 & 3.358330 & 0.002206 & 0.000481 & -0.001042 & 0.005049 & -0.008574 & 0.028016 \\
\hline $\mathrm{Ar}$ & 5.653346 & 0.285876 & 7.109457 & 0.110933 & 0.107269 & 0.082829 & 0.111556 & 0.083046 & 0.072361 \\
\hline At & 10.231864 & 5.029467 & 10.335698 & 101.943568 & 65.724799 & 100.431138 & 42.396490 & 64.759834 & 98.973900 \\
\hline $\mathrm{Au}$ & 7.046423 & 5.557522 & 6.250947 & 0.009488 & -0.009391 & 0.007173 & 0.012160 & -0.008208 & 0.009718 \\
\hline $\mathrm{Ca}$ & -0.056119 & -3.751012 & 2.903333 & 0.029068 & -0.061974 & 0.019386 & 0.144467 & -0.044556 & 0.020405 \\
\hline $\mathrm{Cs}$ & -1.131332 & -3.247545 & 4.836032 & 0.253516 & -0.454367 & -0.297816 & 0.831822 & 0.542589 & 0.366409 \\
\hline $\mathrm{Eu}$ & -0.499530 & -2.309197 & 3.821139 & 0.002968 & 0.000972 & 0.004096 & 0.000727 & 0.001536 & 0.007045 \\
\hline In & 4.852852 & -1.208854 & 6.795223 & 0.064093 & 0.289369 & 0.072830 & 1.335119 & 0.335702 & 0.088999 \\
\hline $\mathrm{Na}$ & 0.658291 & -3.411026 & -0.144969 & 0.000114 & -0.000075 & 0.000066 & 0.001038 & -0.000799 & 0.000962 \\
\hline $\mathrm{Nu}$ & 0.459991 & -2.658697 & 3.484752 & 0.007755 & -0.000608 & 0.001160 & 0.001553 & -0.000336 & 0.004719 \\
\hline $\mathrm{Nz}$ & -1.329904 & -7.952151 & 7.910255 & 0.015252 & 0.034933 & 0.015391 & 0.140454 & 0.058358 & 0.036803 \\
\hline Ok & 3.107407 & -5.000841 & -4.587389 & 1.822874 & -1.538042 & -1.838730 & 1.317255 & 1.561148 & 1.884001 \\
\hline $\mathrm{Pa}$ & -1.923772 & 4.589783 & -10.300427 & 0.009299 & 0.001117 & -0.000448 & 0.002121 & -0.000067 & 0.005219 \\
\hline $\mathrm{Ph}$ & 9.423451 & -5.530138 & -11.523673 & 0.316389 & -0.337266 & -0.192008 & 0.370821 & 0.208181 & 0.131048 \\
\hline $\mathrm{Sa}$ & -1.189878 & -1.174240 & -0.809045 & 0.015218 & -0.013617 & -0.003420 & 0.015430 & 0.003466 & 0.005410 \\
\hline So & -0.644017 & -3.150603 & 4.346639 & 0.002358 & 0.002195 & -0.000185 & 0.002151 & -0.000177 & 0.000253 \\
\hline $\mathrm{Sr}$ & -2.860888 & -7.064668 & 1.463571 & 0.615256 & 1.043353 & -0.941995 & 1.777408 & -1.602858 & 1.452819 \\
\hline $\mathrm{Su}$ & 0.285162 & -5.332412 & 4.302467 & 0.128464 & -0.464367 & 0.020302 & 1.736351 & -0.075708 & 0.016525 \\
\hline
\end{tabular}

${ }^{\text {a }}$ Plate abbreviations are listed in Table 1.

${ }^{\mathrm{b}}$ Units for $\omega$ are $10^{-3} \mathrm{rads} / \mathrm{Myr}$ with the $\mathrm{X}, \mathrm{Y}, \mathrm{Z}$ axes being parallel to $\left(0^{\circ} \mathrm{N}, 0^{\circ} \mathrm{E}\right),\left(0^{\circ} \mathrm{N}, 90^{\circ} \mathrm{E}\right)$, and $90^{\circ} \mathrm{N}$, respectively.

${ }^{\mathrm{c}}$ These six columns give the indicated elements of the covariance matrix $\left(10^{-6} \mathrm{rads}^{2} / \mathrm{Myr}^{2}\right)$. 
Table 4. Relative Angular Velocities for Adjacent Plate Pairs

\begin{tabular}{|c|c|c|c|c|c|c|c|}
\hline Plate Pair $^{\mathrm{a}}$ & $\lambda^{\mathrm{b}},{ }^{\circ} \mathrm{N}$ & $\phi^{\mathrm{b}},{ }^{\circ} \mathrm{E}$ & $\begin{array}{c}\omega, \\
\operatorname{deg} / \mathrm{Myr}\end{array}$ & $\sigma_{\mathrm{maj}}^{\mathrm{c}}$ & $\sigma_{\min }{ }^{c}$ & $\zeta{ }^{\mathrm{d}}$ deg & $\begin{array}{c}\sigma_{\omega}, \mathrm{deg} / \\
\mathrm{Myr}\end{array}$ \\
\hline $\mathrm{Am}-\mathrm{Cs}$ & 10.45 & 113.39 & 0.090 & 56.7 & 7.5 & 21 & 0.113 \\
\hline $\mathrm{Am}-\mathrm{Eu}$ & 44.18 & 158.76 & 0.107 & 33.3 & 6.6 & 88 & 0.100 \\
\hline Am-Ok & 59.14 & 146.80 & 0.648 & 8.1 & 2.0 & 25 & 0.155 \\
\hline $\mathrm{Am}-\mathrm{Ph}$ & 54.74 & 161.65 & 1.168 & 4.2 & 0.9 & 62 & 0.105 \\
\hline $\mathrm{An}-\mathrm{Au}$ & -14.71 & -140.30 & 0.653 & 1.6 & 0.8 & 33 & 0.004 \\
\hline $\mathrm{An}-\mathrm{Nu}$ & -3.25 & 148.10 & 0.128 & 6.8 & 2.7 & 16 & 0.006 \\
\hline An-Nz & -35.12 & 90.90 & 0.453 & 4.0 & 1.0 & -15 & 0.015 \\
\hline $\mathrm{An}-\mathrm{Pa}$ & 65.96 & -85.36 & 0.857 & 0.6 & 0.4 & 88 & 0.011 \\
\hline $\mathrm{An}-\mathrm{Sa}$ & 84.64 & -128.11 & 0.240 & 3.5 & 1.4 & -80 & 0.011 \\
\hline An-So & -28.17 & 115.25 & 0.120 & 5.2 & 3.1 & 16 & 0.007 \\
\hline Ar-At & -26.08 & -133.99 & 0.421 & 52.7 & 1.7 & 36 & 0.853 \\
\hline Ar-Eu & 26.22 & 22.87 & 0.427 & 2.1 & 1.1 & 76 & 0.029 \\
\hline Ar-In & 10.50 & 61.83 & 0.099 & 17.2 & 10.4 & 88 & 0.073 \\
\hline $\mathrm{Ar}-\mathrm{Nu}$ & 31.26 & 29.55 & 0.400 & 1.8 & 1.3 & -85 & 0.030 \\
\hline Ar-So & 21.06 & 28.62 & 0.441 & 1.8 & 1.0 & 55 & 0.029 \\
\hline At-Eu & 26.62 & 34.37 & 0.833 & 20.0 & 0.7 & -5 & 0.871 \\
\hline $\mathrm{Au}-\mathrm{Eu}$ & 12.57 & 46.19 & 0.640 & 1.3 & 0.5 & -52 & 0.005 \\
\hline $\mathrm{Au}-\mathrm{In}$ & -4.38 & 72.04 & 0.409 & 4.7 & 1.7 & 15 & 0.066 \\
\hline $\mathrm{Au}-\mathrm{Pa}$ & 61.40 & 6.16 & 1.080 & 0.6 & 0.4 & 62 & 0.008 \\
\hline $\mathrm{Au}-\mathrm{So}$ & 9.31 & 48.55 & 0.675 & 1.2 & 0.4 & -57 & 0.004 \\
\hline $\mathrm{Au}-\mathrm{Su}$ & 8.64 & 58.16 & 0.743 & 6.5 & 0.9 & -78 & 0.053 \\
\hline $\mathrm{Ca}-\mathrm{Na}$ & 75.45 & -154.55 & 0.180 & 10.9 & 1.3 & 88 & 0.008 \\
\hline $\mathrm{Ca}-\mathrm{Nz}$ & -48.76 & 73.13 & 0.382 & 5.6 & 3.1 & 21 & 0.020 \\
\hline $\mathrm{Ca}-\mathrm{Sa}$ & 52.83 & -66.25 & 0.267 & 5.4 & 1.4 & -5 & 0.021 \\
\hline Cs-Eu & 41.90 & -123.95 & 0.087 & 65.1 & 6.6 & -50 & 0.006 \\
\hline Cs-Ph & 56.57 & 167.80 & 1.123 & 4.2 & 0.9 & 66 & 0.063 \\
\hline $\mathrm{Cs}-\mathrm{Su}$ & 11.95 & 124.19 & 0.148 & 19.0 & 11.6 & 39 & 0.097 \\
\hline Eu-In & -28.56 & -168.38 & 0.357 & 14.4 & 1.1 & -89 & 0.033 \\
\hline Eu-Na & 68.05 & 136.42 & 0.245 & 1.5 & 0.8 & -38 & 0.004 \\
\hline $\mathrm{Eu}-\mathrm{Nu}$ & 18.23 & 159.99 & 0.062 & 9.5 & 3.7 & -17 & 0.005 \\
\hline Eu-Ok & 61.84 & 143.27 & 0.546 & 8.0 & 1.0 & 7 & 0.117 \\
\hline Eu-Pa & 63.49 & -78.34 & 0.904 & 0.6 & 0.4 & 78 & 0.006 \\
\hline Eu-Ph & 55.79 & 162.02 & 1.063 & 2.6 & 0.5 & 45 & 0.040 \\
\hline $\mathrm{Eu}-\mathrm{Su}$ & -8.76 & 104.55 & 0.181 & 5.4 & 2.2 & -4 & 0.078 \\
\hline In-So & 22.78 & 19.46 & 0.362 & 13.1 & 0.9 & 89 & 0.040 \\
\hline $\mathrm{Na}-\mathrm{Nu}$ & -77.90 & -75.23 & 0.213 & 2.0 & 1.2 & 64 & 0.004 \\
\hline $\mathrm{Na}-\mathrm{Ok}$ & 56.68 & 147.01 & 0.305 & 11.8 & 1.7 & 18 & 0.121 \\
\hline $\mathrm{Na}-\mathrm{Pa}$ & 50.38 & -72.11 & 0.755 & 0.6 & 0.4 & -79 & 0.004 \\
\hline $\mathrm{Na}-\mathrm{Sa}$ & 12.89 & -50.43 & 0.171 & 2.7 & 1.3 & -10 & 0.009 \\
\hline $\mathrm{Na}-\mathrm{Sr}$ & -17.59 & 46.07 & 0.305 & 12.2 & 0.9 & -27 & 0.103 \\
\hline $\mathrm{Nu}-\mathrm{Sa}$ & 62.67 & -41.98 & 0.277 & 3.1 & 1.3 & -2 & 0.006 \\
\hline $\mathrm{Nu}-\mathrm{So}$ & -35.49 & 24.02 & 0.085 & 4.9 & 3.1 & -19 & 0.005 \\
\hline $\mathrm{Nz}-\mathrm{Pa}$ & 55.41 & -87.29 & 1.267 & 1.6 & 0.5 & 17 & 0.008 \\
\hline $\mathrm{Nz}-\mathrm{Sa}$ & 52.13 & -91.18 & 0.633 & 3.1 & 1.2 & 8 & 0.009 \\
\hline Ok-Pa & 27.81 & -62.32 & 0.702 & 14.1 & 0.9 & -19 & 0.047 \\
\hline Ok-Ph & 47.58 & 175.21 & 0.538 & 10.5 & 1.7 & 79 & 0.120 \\
\hline $\mathrm{Pa}-\mathrm{Ph}$ & 4.60 & 138.27 & 0.874 & 1.7 & 0.6 & -15 & 0.049 \\
\hline $\mathrm{Pa}-\mathrm{Sr}$ & -45.18 & 85.40 & 0.950 & 3.5 & 0.4 & -78 & 0.105 \\
\hline $\mathrm{Pa}-\mathrm{Su}$ & -55.16 & 102.55 & 1.019 & 5.1 & 0.5 & 3 & 0.048 \\
\hline $\mathrm{Ph}-\mathrm{Su}$ & -59.99 & -1.24 & 1.047 & 6.8 & 0.8 & -72 & 0.035 \\
\hline
\end{tabular}

${ }^{a}$ The first plate rotates counterclockwise relative to the second plate around the listed rotation pole. Plate abbreviations are given in Table 1 .

${ }^{\mathrm{b}}$ Location of rotation pole, $\lambda$ is latitude and $\phi$ is longitude in decimal degrees.

${ }^{\mathrm{c}}$ Two-dimensional 1-sigma lengths in degrees of the semimajor $\sigma_{\text {maj }}$ and semiminor $\sigma_{\min }$ axes of the pole error ellipse.

${ }^{d}$ The parameter $\zeta$ is the azimuth of the semimajor ellipse axis in degrees clockwise from north.

misfit to the data for a given plate, as described by Ward [1990] and Mao [1998]. Relative angular velocities for pairs of adjacent plates are then derived by differencing the ITRF-97 angular velocities, with appropriate error propagation (Table 4). Tables 2, 3, and 4 constitute REVEL-2000.

[13] One advantage of our approach is that GPS site velocities can easily be compared to independent space geodetic data from SLR, VLBI, and DORIS, whose solutions are also available in ITRF-97. In addition, for plates with limited GPS data it is straightforward to incorporate data from these other techniques [e.g., Norabuena et al., 1998, 1999]. (For these sites we use the ITRF-97 SINEX velocity and error solutions available from http://
lareg.ensg.ign.fr/ITRF.) We use these additional data for Arabia, Anatolia, and Nazca. One disadvantage of our approach is that three steps are required to derive a relative angular velocity (namely, transform coordinate time series to ITRF-97 and derive site velocities; derive ITRF-97 plate angular velocities by inverting the site velocities; and difference these angular velocities to derive the relative angular velocities of adjacent plates), whereas it is possible to go directly from loosely constrained (pre-ITRF) position estimates to a plate velocity-minimized frame, deriving a relative angular velocity in only two steps [e.g., Kogan et al., 2000].

[14] A plot of site velocities relative to ITRF-97 (Figure 4) superficially resembles the velocity field predicted by the 
ETG 11 - 12 SELLA ET AL.: REVEL-RECENT PLATE VELOCITIES FROM SPACE GEODESY

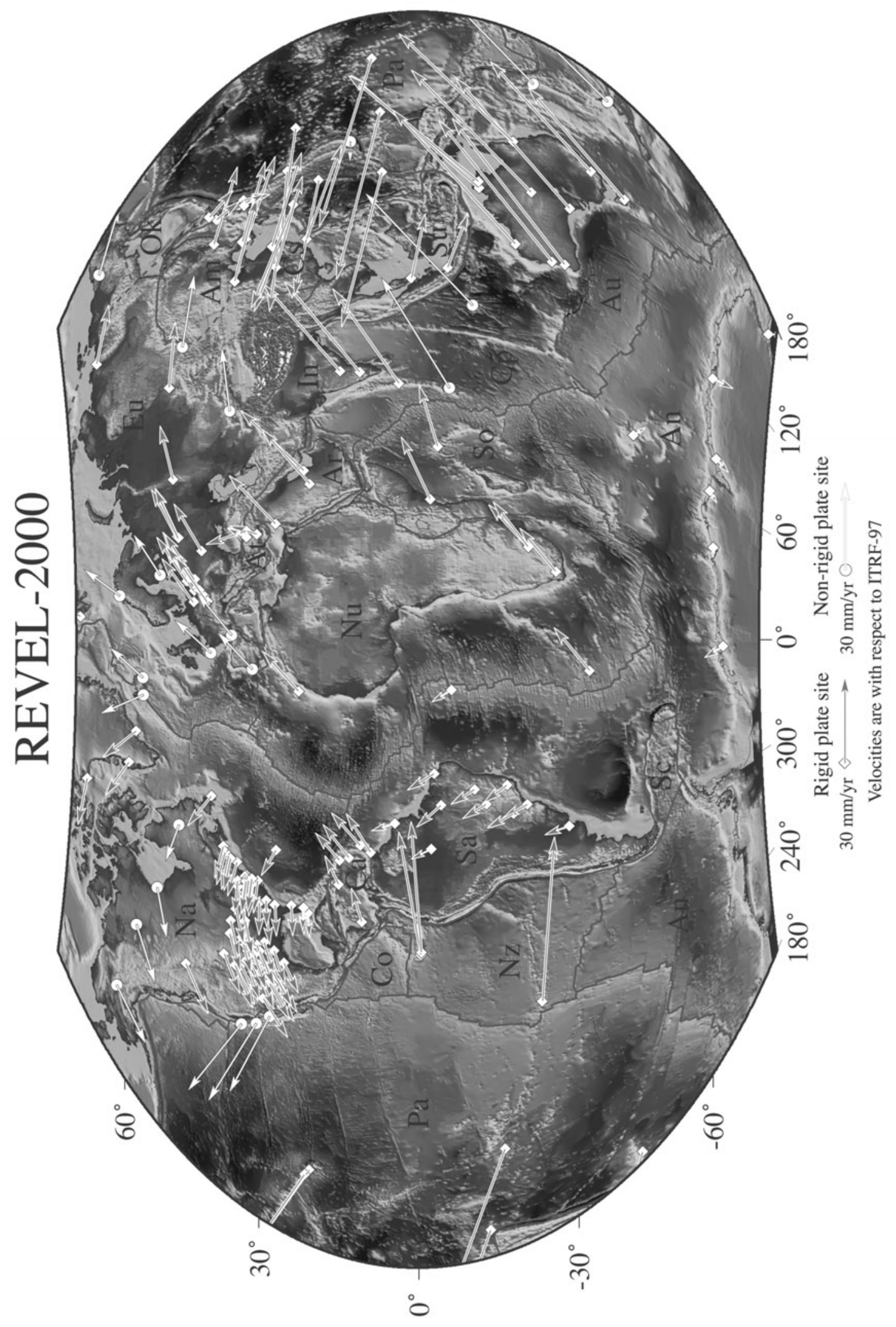

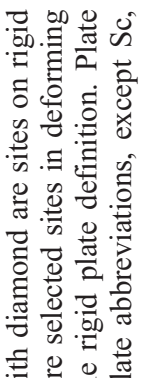

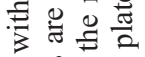

จิ

品

일

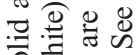

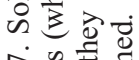

市造㐘

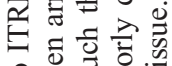

원 0

舫.

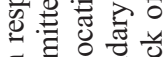

들 0 음

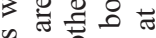

氖范

응

.

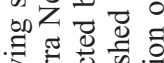

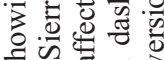

की

응

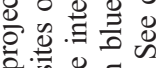

远言

恶 影号

可额 不

के

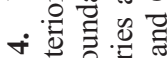

$0 . \Xi \frac{\pi}{3}$

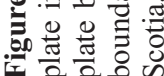


Table 5. Comparison of Relative Angular Velocities for Selected Plate Pairs

\begin{tabular}{|c|c|c|c|c|c|c|c|c|}
\hline Plate Pair ${ }^{\mathrm{a}}$ & $\phi,{ }^{\mathrm{b}} \mathrm{N}$ & $\lambda,{ }^{\mathrm{b}} \mathrm{E}$ & $\omega, \mathrm{deg} / \mathrm{Myr}$ & $\sigma_{\mathrm{maj}}{ }^{\mathrm{c}}$ & $\sigma_{\min }{ }^{\mathrm{c}}$ & $\zeta,^{\mathrm{d}} \mathrm{deg}$ & $\begin{array}{c}\sigma_{\omega}, \\
\operatorname{deg} / \mathrm{Myr}\end{array}$ & Author (Type of Data ${ }^{e}$ ) \\
\hline $\mathrm{Ar}-\mathrm{Nu}$ & 31.26 & 29.55 & 0.400 & 1.8 & 1.3 & -85 & 0.030 & REVEL-2000 \\
\hline $\mathrm{Ar}-\mathrm{Nu}$ & 31.50 & 23.00 & 0.403 & 3.5 & 1.5 & -74 & 0.045 & Chu and Gordon [1998] (MM) ${ }^{\mathrm{f}}$ \\
\hline $\mathrm{Ar}-\mathrm{Nu}$ & 32.59 & 23.70 & 0.400 & - & - & - & - & Jestin et al. [1994] (MM, TA $)^{\mathrm{g}}$ \\
\hline Au-An & 14.71 & 39.70 & 0.653 & 1.6 & 0.8 & -33 & 0.004 & REVEL-2000 \\
\hline $\mathrm{Au}-\mathrm{An}$ & 10.92 & 41.60 & 0.660 & - & - & - & - & Bouin and Vigny [2000] (GP) \\
\hline $\mathrm{Au}-\mathrm{An}$ & 11.89 & 42.82 & 0.650 & 3.1 & 1.0 & -35 & 0.007 & Conder and Forsyth [2000] (MM, TA) \\
\hline $\mathrm{Au}-\mathrm{An}$ & 9.80 & 43.20 & 0.650 & 4.4 & 2.6 & 20 & 0.01 & Larson et al. [1997] (GPS) ${ }^{i}$ \\
\hline $\mathrm{Au}-\mathrm{An}$ & 13.20 & 38.20 & 0.650 & 1.3 & 1.0 & -63 & 0.01 & DeMets et al. [1994] (ES, MM, TA) \\
\hline $\mathrm{Ca}-\mathrm{Na}$ & 75.45 & -154.55 & 0.180 & 10.9 & 1.3 & 88 & 0.008 & REVEL-2000 \\
\hline $\mathrm{Ca}-\mathrm{Na}$ & 69.10 & -136.70 & 0.192 & 31.3 & 4.8 & -7 & 0.036 & DeMets et al. [2000] (GP) \\
\hline $\mathrm{Ca}-\mathrm{Na}$ & 64.90 & -109.50 & 0.214 & 14.6 & 1.5 & -35 & 0.030 & DeMets et al. [2000] (GP, MM, TA) \\
\hline $\mathrm{Ca}-\mathrm{Na}$ & 68.40 & -126.30 & 0.210 & - & - & - & - & Deng and Sykes [1995] (ES) \\
\hline $\mathrm{Ca}-\mathrm{Na}$ & 74.30 & 153.90 & 0.100 & 24.7 & 2.6 & -52 & 0.030 & DeMets et al. [1994] (ES, MM, TA) \\
\hline $\mathrm{Ca}-\mathrm{Sa}$ & 52.83 & -66.25 & 0.267 & 5.4 & 1.4 & -5 & 0.021 & REVEL-2000 \\
\hline $\mathrm{Ca}-\mathrm{Sa}$ & 61.90 & -75.70 & 0.229 & - & - & - & - & Perez et al. [2001] (GP) \\
\hline $\mathrm{Ca}-\mathrm{Sa}$ & 51.50 & -65.70 & 0.272 & 6.1 & 1.9 & -9 & 0.023 & Weber et al. [2001] (GP) \\
\hline $\mathrm{Ca}-\mathrm{Sa}$ & 50.00 & -65.30 & 0.180 & 14.9 & 4.3 & -2 & 0.030 & DeMets et al. [1994] (ES, MM, TA) \\
\hline $\mathrm{Cs}-\mathrm{Eu}$ & 41.90 & -123.95 & 0.087 & 65.1 & 6.6 & -50 & 0.006 & REVEL-2000 \\
\hline $\mathrm{Cs}-\mathrm{Eu}$ & 61.20 & 142.00 & 0.206 & 23.3 & 2.2 & 39 & 0.123 & Heki et al. [1999] (GP) \\
\hline $\mathrm{Cs}-\mathrm{Eu}$ & 64.84 & 156.74 & 0.120 & - & - & - & - & Holt et al. [2000] (GP, QF) \\
\hline $\mathrm{Cs}-\mathrm{Eu}$ & -5.10 & 109.00 & -0.190 & 6.2 & 0.7 & -78 & 0.010 & Peltzer and Saucier [1996] (ES) \\
\hline Eu-Ok & 61.84 & 143.27 & 0.546 & 8.0 & 1.0 & 7 & 0.117 & REVEL-2000 \\
\hline Eu-Ok & 53.02 & 142.09 & 0.405 & 13.5 & 3.4 & -72 & - & Seno et al. [1996] (ES) \\
\hline $\mathrm{Eu}-\mathrm{Na}$ & 68.05 & 136.42 & 0.245 & 1.5 & 0.8 & -38 & 0.004 & REVEL-2000 \\
\hline $\mathrm{Eu}-\mathrm{Na}$ & 74.30 & 123.00 & 0.231 & 1.8 & 1.4 & 16 & 0.008 & Kogan et al. [2000] (GP) \\
\hline $\mathrm{Eu}-\mathrm{Na}$ & 78.50 & 122.00 & 0.230 & 8.2 & 4.9 & -8 & 0.030 & Argus and Heflin [1995] (GP) \\
\hline $\mathrm{Eu}-\mathrm{Na}$ & 62.40 & 135.80 & 0.310 & 4.1 & 2.3 & -11 & 0.010 & DeMets et al. [1994] (ES, MM, TA) \\
\hline In-Eu & 28.56 & 11.62 & 0.357 & 14.4 & 1.1 & 89 & 0.033 & REVEL-2000 \\
\hline In-Eu & 25.60 & 11.10 & 0.440 & - & - & - & 0.030 & Paul et al. [2001] (GP) \\
\hline $\mathrm{In}-\mathrm{Eu}$ & 29.78 & 7.51 & 0.353 & - & - & - & - & Holt et al. [2000] (GP) \\
\hline In-Eu & 24.40 & 17.70 & 0.510 & 8.8 & 1.8 & -79 & 0.050 & DeMets et al. [1994] (ES, MM, TA) \\
\hline $\mathrm{Na}-\mathrm{Pa}$ & 50.38 & -72.11 & 0.755 & 0.6 & 0.4 & -79 & 0.004 & REVEL-2000 \\
\hline $\mathrm{Na}-\mathrm{Pa}$ & 51.50 & -73.70 & 0.765 & 2.0 & 1.0 & -85 & 0.016 & DeMets and Dixon [1999] (GS) \\
\hline $\mathrm{Na}-\mathrm{Pa}$ & 50.00 & -76.10 & 0.777 & 0.8 & 0.6 & 65 & 0.007 & DeMets and Dixon [1999] (MM, TA) \\
\hline $\mathrm{Na}-\mathrm{Pa}$ & 49.60 & -84.30 & 0.830 & 2.0 & 1.0 & -86 & 0.020 & Larson et al. [1997] (GP) \\
\hline $\mathrm{Na}-\mathrm{Pa}$ & 49.10 & -73.00 & 0.790 & 4.1 & 2.2 & -83 & 0.030 & Argus and Heflin [1995] (GP) \\
\hline $\mathrm{Na}-\mathrm{Pa}$ & 48.70 & -78.20 & 0.750 & 1.3 & 1.2 & -61 & 0.010 & DeMets et al. [1994] (ES, MM, TA) \\
\hline $\mathrm{Na}-\mathrm{Sa}$ & 12.89 & -50.43 & 0.171 & 2.7 & 1.3 & -10 & 0.009 & REVEL-2000 \\
\hline $\mathrm{Na}-\mathrm{Sa}$ & 12.00 & -50.60 & 0.130 & 6.8 & 3.0 & -19 & 0.03 & Dixon and Mao [1997] (GP) \\
\hline $\mathrm{Na}-\mathrm{Sa}$ & 11.10 & -53.30 & 0.290 & 76.6 & 3.9 & 37 & 0.08 & Larson et al. [1997] (GP) ${ }^{\mathrm{i}}$ \\
\hline $\mathrm{Na}-\mathrm{Sa}$ & 6.50 & -55.60 & 0.280 & 8.3 & 7.4 & -55 & 0.12 & Argus and Heflin [1995] (GP) \\
\hline $\mathrm{Na}-\mathrm{Sa}$ & 16.30 & -58.10 & 0.150 & 5.9 & 3.7 & -9 & 0.01 & DeMets et al. [1994] (ES, MM, TA) \\
\hline Nu-So & -35.49 & 24.02 & 0.085 & 4.9 & 3.1 & -19 & 0.005 & REVEL-2000 \\
\hline $\mathrm{Nu}-\mathrm{So}$ & -27.30 & 36.20 & 0.089 & 6.9 & 5.5 & 38 & 0.004 & Chu and Gordon [1999] (MM, TA) ${ }^{\mathrm{f}}$ \\
\hline Nu-So & -55.73 & 19.76 & 0.052 & - & - & - & - & Jestin et al. [1994] $(\mathrm{TA}, \mathrm{MM})^{\mathrm{g}}$ \\
\hline $\mathrm{Ph}-\mathrm{Pa}$ & -4.60 & -41.73 & 0.874 & 1.7 & 0.6 & 15 & 0.049 & REVEL-2000 \\
\hline $\mathrm{Ph}-\mathrm{Pa}$ & -7.51 & -42.81 & 1.009 & - & - & - & - & Wei and Seno [1998] (ES) \\
\hline $\mathrm{Ph}-\mathrm{Pa}$ & -1.24 & -45.81 & 1.000 & - & - & - & - & Seno et al. [1993] (ES) \\
\hline
\end{tabular}

${ }^{a}$ The first plate rotates counterclockwise relative to the second plate around the listed rotation pole. Plate abbreviations are given in Table 1.

${ }^{\mathrm{b}}$ Location of rotation pole, $\lambda$ is latitude and $\phi$ is longitude in decimal degrees.

${ }^{\mathrm{c}}$ Two-dimensional 1-sigma lengths in degrees of the semimajor $\sigma_{\text {maj }}$ and semiminor $\sigma_{\min }$ axes of the pole error ellipse.

${ }^{\mathrm{d}}$ The parameter $\zeta$ is the azimuth of the semimajor ellipse axis in degrees clockwise from north.

${ }^{\mathrm{e}} \mathrm{ES}$, earthquake slip vectors; GP, GPS; MM, marine magnetic; QF, Quaternary fault slip rates; TA, transform azimuth; VL, VLBI.

${ }^{\mathrm{f}}$ Estimated from their covariance matrix.

${ }^{\mathrm{g}}$ The best enforced closure solution and velocity reduced by $4.4 \%$ to match Hilgen timescale.

h Based on 1 Ma "Jaramillo event."

${ }^{\mathrm{i}}$ Originally published as reverse plate pair.

NUVEL-1 No-Net-Rotation (NUVEL-1-NNR) velocity field [Argus and Gordon, 1991] or its updated version, NUVEL-1ANNR [DeMets et al., 1994], because of the way ITRF is defined. Inasmuch as the NUVEL-1A geologic model may be a biased representation of Recent plate motions for some plate pairs, as described earlier, it may be inappropriate to use the NNR reference frame based on NUVEL-1A for geodetic observations. Recent versions of the ITRF are believed to be free of biases associated with a particular plate motion model [Sillard et al., 1998]. However, to be conservative, in our approach, ITRF-97 is treated as an arbitrary initial reference frame, differenced out in the subsequent calculation of relative angular velocities. Hence any bias in the ITRF should not influence our results. The success of our approach can be assessed in two ways:

1. The orientation of residual velocities on a given plate should be random, and their magnitude should be similar to the site velocity uncertainty.

2. Independent geologic and geodetic estimates along a given plate boundary should agree with the predictions of our angular velocities.

[15] Both tests are applied in this paper and suggest that for the most part our approach is valid. Briefly, residual velocities for most 


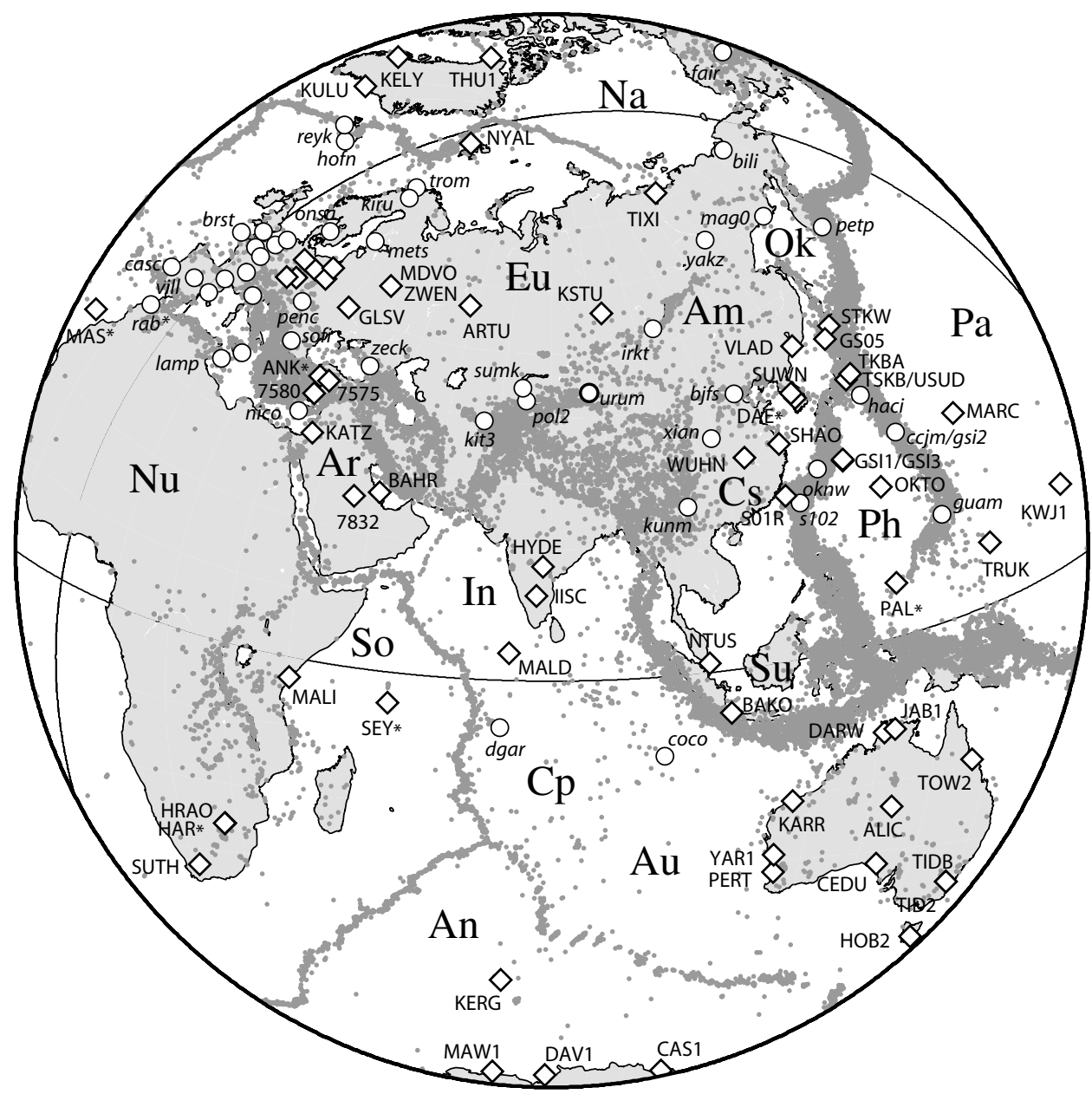

Figure 5. Lambert azimuthal equal-area projection showing all space geodetic site locations used in this study for the area around the Indian Ocean. Earthquake epicenters (magnitude $>4.5$, depth $<50 \mathrm{~km}$, between 1973 and 2000) suggest plate boundaries. Sites labeled with diamonds and upper case letters are assumed to lie on the rigid plate interior and are used to define the plate's angular velocity (Table 2). Sites labeled with circles and lowercase letters are not used in the rigid plate definition (Table 2). See Table 1 for plate abbreviations, except Cp, Capricorn. For clarity, site names in some areas have been omitted, e.g., Europe.

plates are randomly oriented in azimuth and have magnitudes that are about the level expected given observational error. Exceptions are explicitly discussed below.

[16] As our new velocity data incorporate a longer time span, additional sites, and improved analytical techniques, the angular velocities presented here are more precise than our previous published estimates for individual plate pairs. However, the major conclusions of those studies remain unchanged [Dixon and Mao, 1997; Dixon et al., 1998; DeMets and Dixon, 1999; Norabuena et al., 1999; Dixon et al., 2000a, 2000b; Weber et al., 2001].

\section{Results}

[17] Our results are summarized in Tables 1, 2, 3, and 4 and Figures 4-21. Site locations are displayed in the global map figures (Figures 5, 13, and 17), along with seismicity (magnitude $>4.5$ and depth $<50 \mathrm{~km}$ ) for the period 1973-2000, from the National Earthquake Information Center (http://neic.usgs. gov), which generally outlines the appropriate plate boundary. Table 1 lists the individual site velocities in ITRF-97 grouped by plate, as well as site positions, total time span of data, number of data (number of daily position estimates), weighted root mean square scatter (WRMS) of the data about a best fit line (an indicator of data quality), and the residual velocities predicted from the best fitting ITRF-97 angular velocity for each plate (Table 2).

[18] Table 2 lists the best fitting angular velocity for each plate or block with respect to ITRF-97, the mean rate and RMS of the velocity residuals, and the $x^{2}$ per degree of freedom. Table 3 lists the corresponding Cartesian coordinates and covariance matrix. Table 4 lists the relative angular velocities for pairs of plates sharing a common boundary. Table 5 compares a subset of these angular velocities to some previously published values.

\section{Discussion}

\subsection{Plate Rigidity and Error Model}

[19] Rigid plate behavior on timescales of several million years is demonstrated by the success of rigid plate geologic models, and the exceptions are well described and spatially limited [Gordon, 1998]. Space geodesy can test the assumption of rigid plates on shorter timescales [Argus and Gordon, 1996; Dixon et al., 1996]. The existence of intraplate seismicity such as the New Madrid seismic zone [Nuttli, 1973; Schweig and Ellis, 1994; Weber et al., 1998; Newman et al., 1999] argues that some local intraplate deformation must occur. Plate rigidity over several years at the level of a few millimeters per year is suggested by the generally good agreement between geologic models and space geodetic 
estimates of plate motion [Smith et al., 1990]. On the other hand, the spatial sampling afforded by techniques such as VLBI and SLR is limited, and until recently, the precision of GPS, which in principle can provide better spatial sampling, has also been limited. Dixon et al. [1996] noted that with the data then available, GPS data lacked the sensitivity to detect a clear postglacial rebound signal and provided only a crude upper limit to plate rigidity, as apparent deviations from a rigid plate model (e.g., the velocity residuals in Tables 1 and 2) mainly reflect GPS velocity uncertainties rather than true nonrigid plate processes. While the current data set is a significant improvement relative to the data available in 1996, and apparently has some sensitivity to postglacial rebound (see section 4.2), the same limitation still applies, although we can now place a tighter upper bound on plate rigidity from the GPS data. Useful constraints are available in the current data set for the North American, Eurasian, and possibly Australian plates. For the remaining plates, the number of data is too small to derive rigorous estimates of plate rigidity, although we note that the velocity data fit a rigid plate model at about the expected level for most of the plates studied (Figure $2 b$ ).

[20] Using all 84 available sites to define stable North America, comprising sites on the plate interior that have observations spanning as short as 2.0 years, the mean rate residual is 0.99 $\mathrm{mm} / \mathrm{yr}$. Below, we describe a 64-site solution that eliminates sites most sensitive to postglacial rebound, resulting in a mean rate residual of $0.86 \mathrm{~mm} / \mathrm{yr}$. In either case the residual magnitude is roughly the same as the GPS velocity error, suggesting that the rigid plate model is appropriate and that the residuals likely reflect GPS observation errors rather than nonrigid plate processes, as found in earlier studies [e.g., Dixon et al., 1996]. Since GPS velocity errors are a strong function of total observing time, one way to check this conclusion is to restrict the data set to sites with longer time spans and to investigate the effect on residuals. The residual magnitudes as a function of mean observing time are consistent with the error model illustrated in Figure 3. For example, if we base the angular velocity estimate for North America on the 36 sites in our database that are not sensitive to postglacial rebound and have time spans of 4 years or longer, the mean rate residual is $0.71 \mathrm{~mm} / \mathrm{yr}$, compared to $0.86 \mathrm{~mm} / \mathrm{yr}$ for the larger data set, supporting the inference that the rate residuals reflect GPS velocity errors rather than nonrigid plate processes.

[21] Rate residuals are positively biased (Argus and Gordon [1996] and Argus et al. [1999] give a complete discussion) implying that the actual level of plate nonrigidity could be higher than the mean rate residual. If we arbitrarily assume that an upper bound on plate rigidity is 3 times the mean rate residual, then the GPS data set composed of 4 years or longer time series suggests that the stable interior of North America is rigid to better than 2.2 $\mathrm{mm} / \mathrm{yr}$. Presumably, this bound will be better constrained in future years as GPS velocity uncertainties decline further and new sites are added. Of the 19 plates and continental blocks examined here, all but three (Anatolia, Arabia, Nazca) have mean rate residuals of $1.8 \mathrm{~mm} / \mathrm{yr}$ or less (Table 2). These three plates or blocks have a very limited distribution of sites, suggesting that their angular velocities are not well determined. The main point for this paper is that the velocity residuals, whether reflecting true nonrigid plate processes, local site effects, or (our preferred explanation) GPS velocity uncertainties, are small enough for the rigid plate approximation to be assumed valid for the data set under consideration.

[22] The relatively uniform nature of space geodetic data makes it feasible to apply a consistent error model, facilitating statistical tests of plate rigidity. If our error model for GPS velocities is approximately correct, $\chi^{2}$ per degree of freedom (hereafter $\chi_{v}^{2}$ ) for the individual plates should approximately equal 1.0 , provided that the data fit the rigid plate model, and that the data set is large enough to be statistically representative. The latter criterion is met for the North American plate $\left(128\right.$ data; $\left.\chi_{v}^{2}=1.05\right)$ and the
Eurasian plate ( 30 data; $\chi_{v}^{2}=1.02$ ). However, the majority of plates tested also appear to approximately satisfy this "rule of thumb," despite the relatively sparse data (Figure $2 b$ ). All but three plates or blocks (Amuria, Australia, Okhotsk) have $x_{v}^{2}<2.0$, and two of these (Amuria and Okhotsk) are probably related to limited data (Table 2). Since the error model is derived independently of any rigid plate criteria, this tends to confirm the joint hypothesis that the great majority of plates and blocks tested are rigid within the velocity uncertainty, that the sites used to define the various plates lie on the rigid portion of the plate interior, and that the error model is approximately correct. The fact that $\chi_{v}^{2}$ for North America and Eurasia is slightly larger than 1.00 might reflect the fact that we have neglected random walk noise. However, the effect is small and is ignored here.

[23] For several plates the number of space geodetic data is small enough that the solutions are sensitive to outliers at one or two sites, the statistical effect of which is diminished when a large number of sites is available. For example, Amuria and Okhotsk, with only three sites and five sites, respectively, have $\chi_{v}^{2}=2.3$ and 4.4 , suggesting that these data are not well fit by the rigid plate model. Possible explanations include that (1) the number of data on the plate are too small to be statistically representative; (2) one or more site velocities have a systematic error not reflected in the error model; (3) our choice of sites is inappropriate, and one or more sites lie in a deforming boundary zone rather than the rigid plate or block interior; and (4) the plate is not rigid. We suspect that explanations 1,2, and 3 together explain such misfits, reflecting limitations in available data.

\subsection{Glacial Isostatic Adjustment}

[24] The lithosphere in North America, Eurasia, and Antarctica is isostatically adjusting from mass loading and unloading of the last glaciation, reflecting the delayed response associated with viscous flow in the Earth's mantle. This adjustment imparts both vertical and horizontal motions to the surface velocity field [e.g., Peltier, 1998a] and needs to be considered when using geodetic data to derive plate motion models because the velocity effects are not representative of longer-term motions. That part of the surface velocity field due to glacial isostatic adjustment (GIA) is a function of both ice loading history and mantle viscosity structure, neither of which is well known, so the corresponding model predictions can vary significantly [e.g., Argus et al., 1999; Mitrovica et al., 2000]. However, most models agree on the region of maximum glacial isosatic effect, even if they do not agree on the magnitude or direction of predicted motion. Rather than correcting each site velocity by a specific model prediction, we take a more conservative approach and simply eliminate the subset of sites most likely to be affected by GIA. For North America, maximum GIA-related motion is centered in a broad region around Hudson Bay. We use the ICE-4G model [Peltier, 1994] to define sites with horizontal rates due to GIA $\geq 0.7 \mathrm{~mm} / \mathrm{yr}$ and compare solutions with and without this data subset. Thus we compare a solution for North America using all available data $\left(84\right.$ sites, $\left.\chi_{v}^{2}=1.63\right)$ versus a model that omits 20 sites most affected by GIA (algo, chb1, chur, det1, dubo, flin, kew1, mil1, neb3, nrc1, oro , sag1, sch2, stb1, stp1, vcap, whp1, wis1, yell, you1) giving a 64-site model with $\chi_{v}^{2}=1.05$. Of these 20 sites, 9 exhibit statistically significant uplift (greater than one standard error), and 5 exhibit uplift rates greater than $3.0 \mathrm{~mm} / \mathrm{yr}$ (Table 1). Sites may also subside due to GIA, associated with collapse of the peripheral bulge, but associated horizontal velocities are thought to be small compared to our observational error.

[25] For Eurasia we compared the solution using all available sites (19 sites, $\left.\chi_{v}^{2}=3.73\right)$ versus a solution that omits four sites on the Fennoscandian platform (kiru, mets, onsa, trom) most likely to be affected by GIA. The omitted sites were chosen using the same criteria for horizontal motions as for North America 
[Peltier, 1998b], approximately equivalent to eliminating sites with vertical motion $>3 \mathrm{~mm} / \mathrm{yr}$, as predicted by the uplift model of Davis et al. [1999]. In this case the fit is significantly improved $\left(15\right.$ sites, $\left.\chi_{v}^{2}=1.02\right)$. We conclude that the current GPS velocity field is sensitive to GIA and that correcting for this effect or eliminating sites sensitive to it is important for obtaining accurate plate angular velocity estimates for comparison to geologic values. For Antarctica we cannot do this as all sites are experiencing GIA uplift (see Table 1).

\subsection{Comparison to Geologic and Geodetic Data}

[26] In this section we discuss the criteria for inclusion or omission of key sites by plate (listed alphabetically, except South America, included in the Nazca plate discussion), compare our results to independent data and the NUVEL-1A geologic model [DeMets et al., 1994], and discuss some implications. The discussion below focuses on those plates or blocks where limited available data make the results sensitive to the selection criteria, where differences appear to exist with NUVEL-1A, and on the relative velocities of several of the "new" plates or continental blocks that have not been included in previous global models. We omit discussion of plates or blocks where our data are limited or where our results do not differ significantly from previous publications: Anatolia [Reilinger et al., 1997; McClusky et al., 2000], Okhotsk [Seno et al., 1996; Wei and Seno, 1998], Sierra Nevada [Dixon et al., 2000a], and Sunda [Walpersdorf et al., 1998; Rangin et al., 1999; Chamot-Rooke and Le Pichon, 1999].

4.3.1. Amuria. [27] Angular velocities for the Amurian plate have been reported by Zonenshain and Savostin [1981], Wei and Seno [1998], Heki et al. [1999], and Holt et al. [2000]. Following Heki et al. [1999], we use three sites to define this plate, DAE*, SUWN, and VLAD (Figure 5). The limited geographic distribution results in correspondingly large uncertainties. Site bjfs is not included in our rigid plate definition since it is located in an area of relatively high seismicity and active faulting [Shen et al., 2000] (the 4-site solution including bjfs, $62.64^{\circ} \mathrm{N},-128.76^{\circ} \mathrm{E}$, $0.319^{\circ} / \mathrm{Myr}, \sigma_{\text {maj }}=17.1, \sigma_{\text {min }}=1.3, \sigma_{\omega}=0.036^{\circ} / \mathrm{Myr}$, mean rate residual $(\mathrm{MMR})=1.2 \mathrm{~mm} / \mathrm{yr}$, is similar to the 3 -site solution listed in Table 2 and improves $\chi_{v}^{2}$ from 2.30 to 1.46).

[28] The formation of the Baikal rift reflects relative motion between Amuria and Eurasia [Zonenshain and Savostin, 1981]. Our relative angular velocity predicts south-southeast extension across the rift at $6-7 \mathrm{~mm} / \mathrm{yr}$. At $47.5^{\circ} \mathrm{N}, 106.5^{\circ} \mathrm{E}$, close to where Calais and Amarjargal [2000] measure a velocity of $6.4 \pm 1.6 \mathrm{~mm} / \mathrm{yr}$ at an azimuth of $125 \pm 30^{\circ}$ from continuous GPS data, we predict (Amuria relative to Eurasia) $7.0 \pm 3.2 \mathrm{~mm} / \mathrm{yr}$, $165 \pm 19^{\circ}$.

4.3.2. Antarctica. [29] We define the Antarctic plate using seven sites (Table 1), excluding ohig and palm because of their proximity to the complex plate boundary with the Scotia plate [Pelayo and Wiens, 1989; Klepeis and Lawver, 1996]. The Antarctic-Australia and Antarctic-Pacific boundaries are spreading ridges, and their velocities are well determined in geologic models such as NUVEL-1A. Pacific and Australia velocities are also well determined in REVEL-2000 (Tables 1, 2, and 3). Any difference in velocity between the two models probably indicates real velocity changes. Figures 6 and 7 indicate that the rates and azimuths predicted by the two models for both plate pairs are virtually identical along most of the respective plate boundaries. For Australia-Antarctica, REVEL-2000 and NUVEL-1A agree to better than $0.6 \mathrm{~mm} / \mathrm{yr}$ in rate for $>80 \%$ of the entire plate boundary, implying a remarkable steadiness of plate motion for this plate pair over the last $3 \mathrm{Myr}$.

4.3.3. Arabia. [30] We define the Arabian plate using a combination of two GPS sites (BAHR, KATZ), and one SLR site (7832) (Figure 5). KATZ is within $30 \mathrm{~km}$ of the active Dead Sea fault and thus does not meet our criterion for minimum distance from the plate boundary. However, the amount of strain accumulation is believed to be small here [Pe'eri et al., 2002]. The north striking Dead Sea fault is a left-lateral strike-slip fault separating Arabia and the Sinai block, a small continental block that may move relative to Nubia. Young $(<140$ kyr $)$ offset geomorphic features along the Dead Sea fault at $30.8^{\circ} \mathrm{N}, 35.4^{\circ} \mathrm{E}$ indicate a slip rate of $4 \pm 2 \mathrm{~mm} / \mathrm{yr}$ at a strike of $028^{\circ}$ [Klinger et al., 2000]. Our predicted Arabia-Nubia velocity at this location is very similar, $3.9 \pm 0.8 \mathrm{~mm} / \mathrm{yr}$ at an azimuth of $009 \pm 10^{\circ}$, implying very slow motion of Sinai relative to Nubia.

[31] If motion of Arabia relative to Nubia has been steady over the last few million years, we expect agreement between the REVEL-2000 prediction and the rate of seafloor spreading across the Red Sea. However, measured spreading rates averaged over the last $3 \mathrm{Myr}$ [Chu and Gordon, 1998], as well as the model rates of Jestin et al. [1994] based on a similar time period, are both systematically higher than REVEL-2000 (Figure 8 and Table 5). Similarly, NUVEL-1A's predicted Arabia-Eurasia rate is higher than REVEL-2000 (Figure 9). McClusky et al. [2000] obtained a similar result. These rate differences may reflect gradual slowing of the Arabian plate as it moves north and collides with Eurasia. Associated crustal thickening forms the Zagros [Alavi, 1994] and Caucasus Mountains, increasing gravitational body forces that oppose Arabia's northward motion, perhaps resulting in gradual slowing of Arabian-Eurasia convergence and Red Sea spreading.

4.3.4. Australia. [32] We define Australian plate motion using 11 sites on Australia and Tasmania (Table 1 and Figure 10). Sites noum and auck have velocities relative to stable Australia that are larger than the corresponding velocities of stable interior sites, perhaps reflecting strain accumulation associated with nearby subduction zones, suggesting that they should not be used for the Australian plate definition. The parameter $\chi_{v}^{2}$ is 3.18 for a 13 -site solution that includes noum and auck versus 2.78 for the 11 -site solution; $\chi_{v}^{2}$ is 3.31 for a 12 -site solution that includes auck. However, even omitting noum and auck results in misfits and a $\chi_{v}^{2}$ that is higher than expected. The velocity residual pattern is very similar to the direction of maximum horizontal compression in the region. Recent compilations of stress data [Hillis and Reynolds, 2000] suggest east-west compression in western Australia, rotating to northwest-southeast compression in southeastern Australia and northeast-southwest compression in northeast Australia (e.g., in vicinity of TOW2) (Figure 10). This may explain why $\chi_{v}^{2}$ for this plate is high. In other words, the rigid plate assumption may not be strictly valid for the continental portion of the plate, and Australia may be undergoing intraplate deformation in response to these compressional stresses. Alternatively, we may have underestimated the site velocity errors for Australia. We prefer the former explanation because the error model gives consistent results for most other plates (Figure $2 b$ ).

[33] Separate Indian and Australian plates have been recognized for some time [Wiens et al., 1985; DeMets et al., 1988], and a Capricorn plate has also been proposed in the central western Indian Ocean with a broad, diffuse boundary [Royer and Gordon, 1997; Gordon et al., 1998]. We are unable to calculate relative angular velocities for plate pairs involving the Capricorn plate because of insufficient geodetic data. However, we can use the $\chi^{2}$ test to evaluate the fit of the velocity data for coco (Cocos Island, on the southern edge of India-Australia boundary), dgar (Diego Garcia, near the inferred boundary between the India and Capricorn plates), HYDE (Hyderbad, India), IISC (Bangalore, India), and MALD (Male, Maldives) to our rigid Australian plate model, with 11 sites and $\chi_{v}^{2}=2.78$ (Figure 5). The $\chi_{v}^{2}$ for the $11+5$ sites is 15.25 , i.e., significantly higher, indicating a very poor fit. If we exclude HYDE, IISC, and MALD (11 + 2 sites), $\chi_{v}^{2}$ drops to 2.74 , consistent with separate Indian and Australian plates (e.g., using the $F$ ratio test of Stein and Gordon [1984]). However, when we exclude either dgar or coco in an $11+1$ site solution for Australia, we get little or no improvement (excluding dgar gives $\chi_{v}^{2}=2.72$, excluding coco gives $\chi_{v}^{2}=2.86$ ). The small change from excluding 

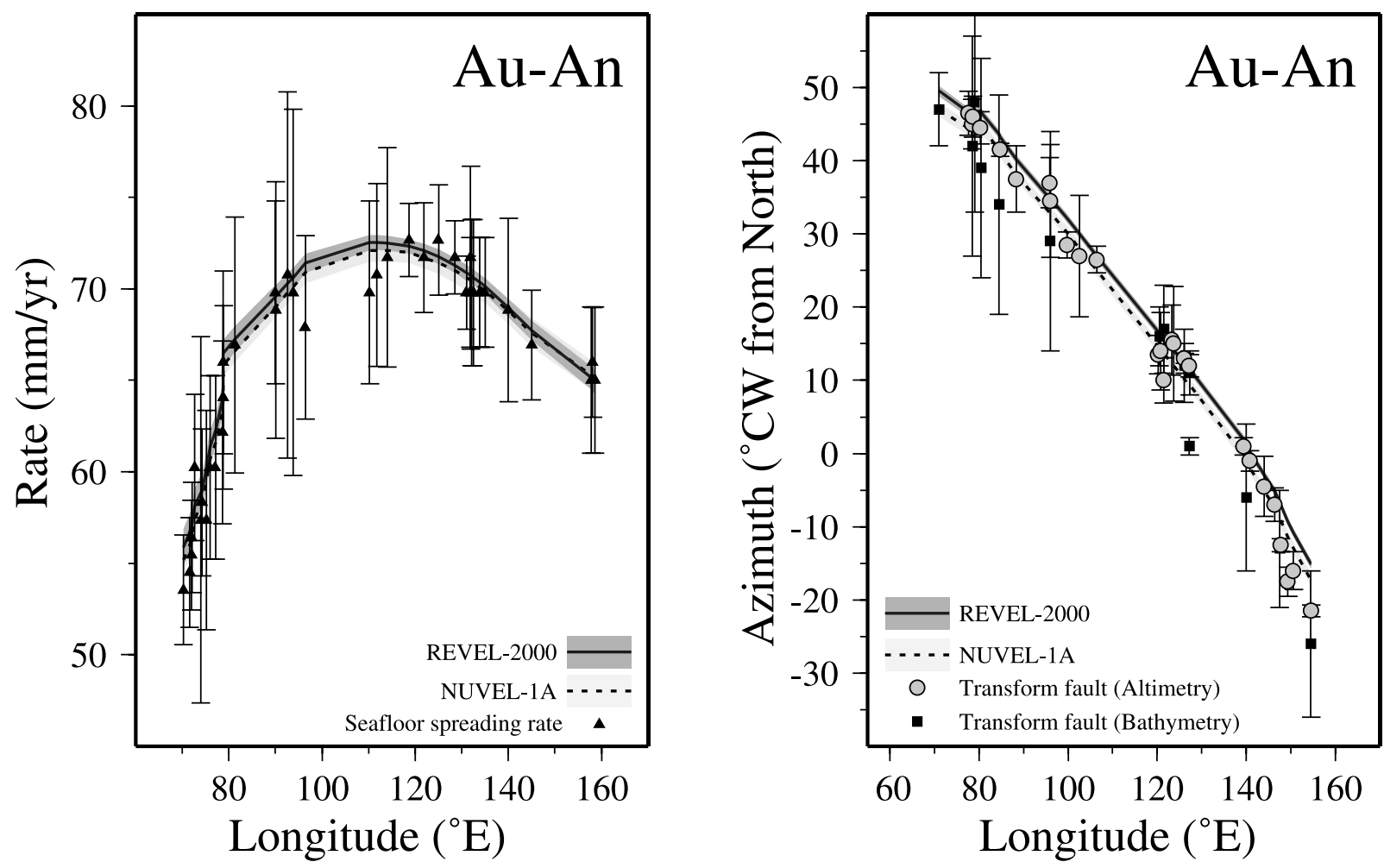

Figure 6. Measured seafloor spreading rates and transform fault azimuths for Australia-Antarctica compared to predicted rate and azimuth at the same location from REVEL-2000 (this study) and NUVEL-1A [DeMets et al., 1994]. Spreading rates are from DeMets et al. [1990] corrected according to DeMets et al. [1994]. Transform azimuths from bathymetric measurements are from DeMets et al. [1990] unless otherwise noted. Transform azimuths from altimetry are from Spitzak and DeMets [1996]. Dark shading indicates \pm 1 standard error for REVEL-2000. Light shading indicates \pm 1 standard error for NUVEL-1A.
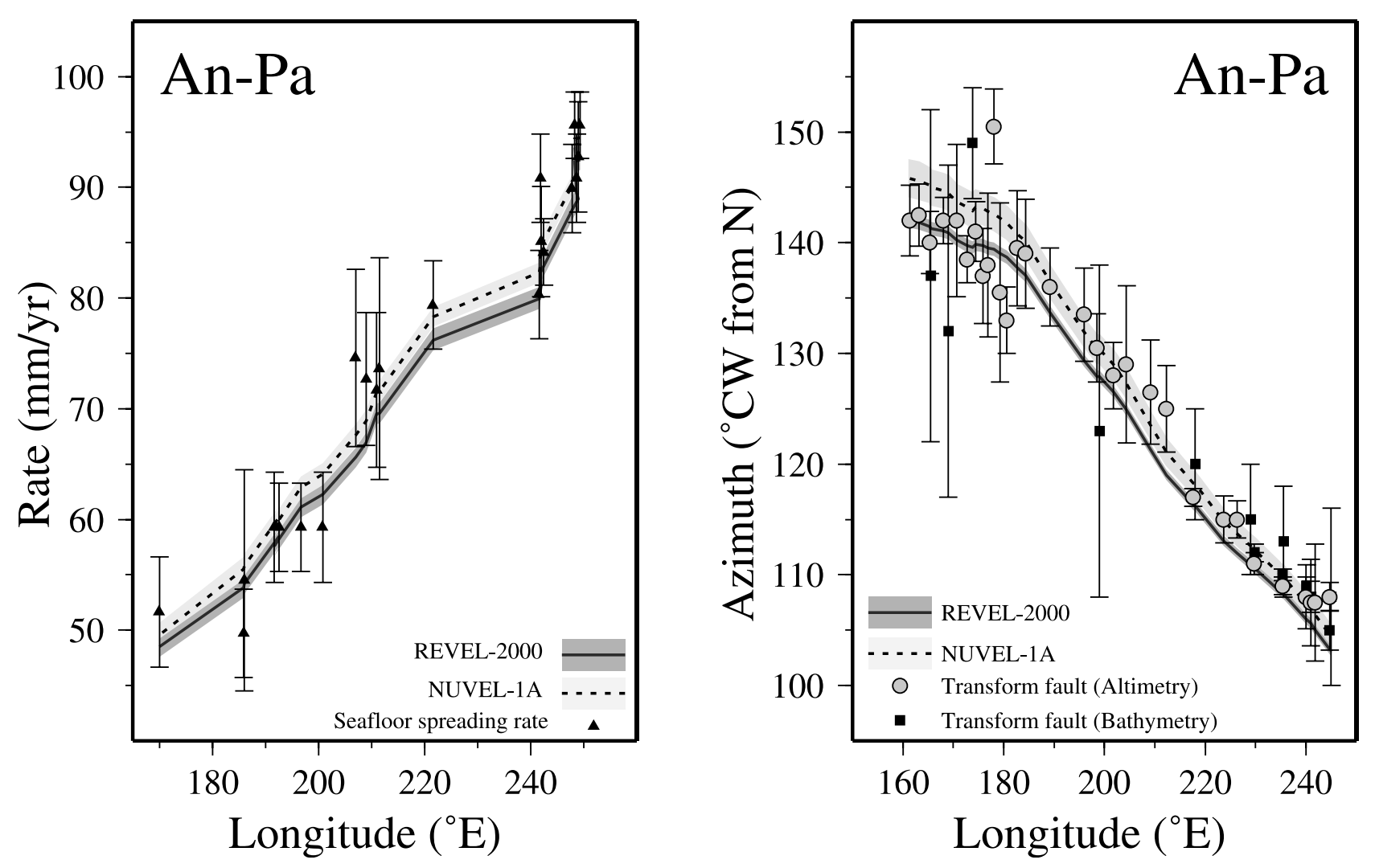

Figure 7. Similar to Figure 6, for Antarctica-Pacific. 

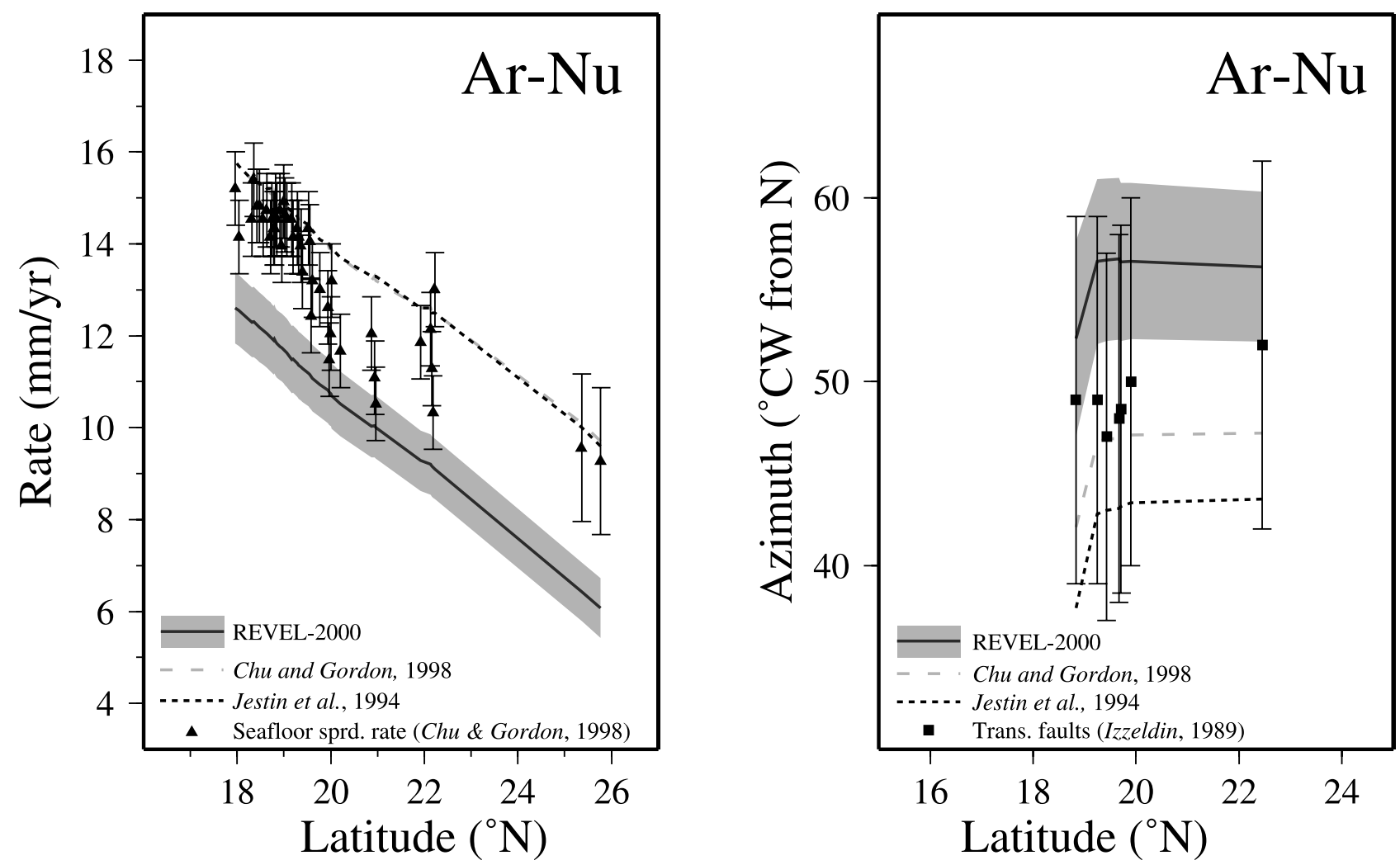

Figure 8. Similar to Figure 6, for Arabia-Nubia. Jestin et al. [1994] pole is their best enforced closure pole (their Table 4) and has been converted to Hilgen timescale by reducing the angular velocity by $4.4 \%$. Transform fault azimuth data of Izzeldin [1989] are only those transform faults that offset at least two marine magnetic anomalies (his Table 1).
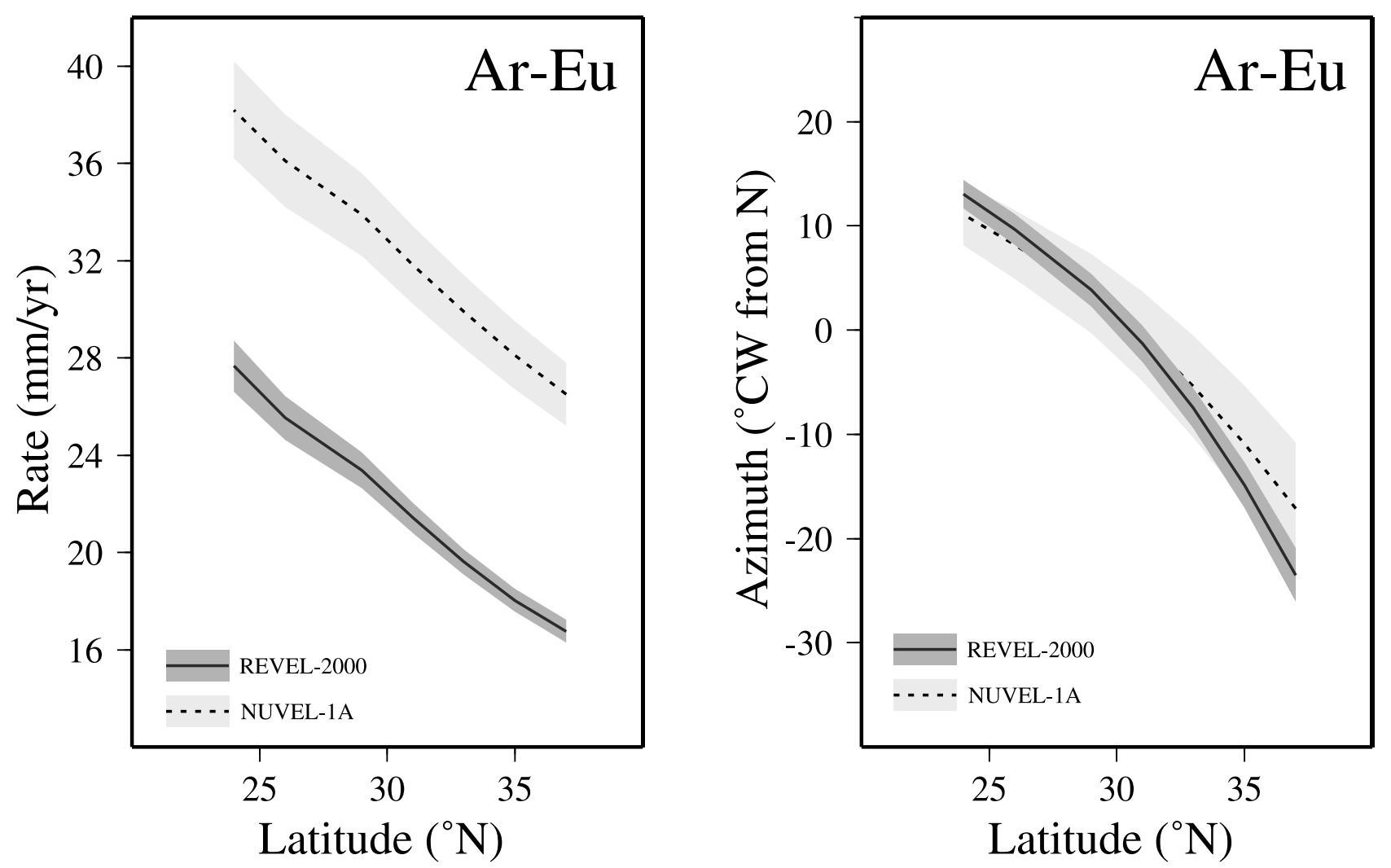

Figure 9. Similar to Figure 6, for Arabia-Eurasia. Site predictions are calculated along a line that approximates the plate boundary, subparallel to the north shore of the Persian Gulf $\left(37^{\circ} \mathrm{N}, 44^{\circ} \mathrm{E}\right.$ to $\left.24^{\circ} \mathrm{N}, 62^{\circ} \mathrm{E}\right)$. 


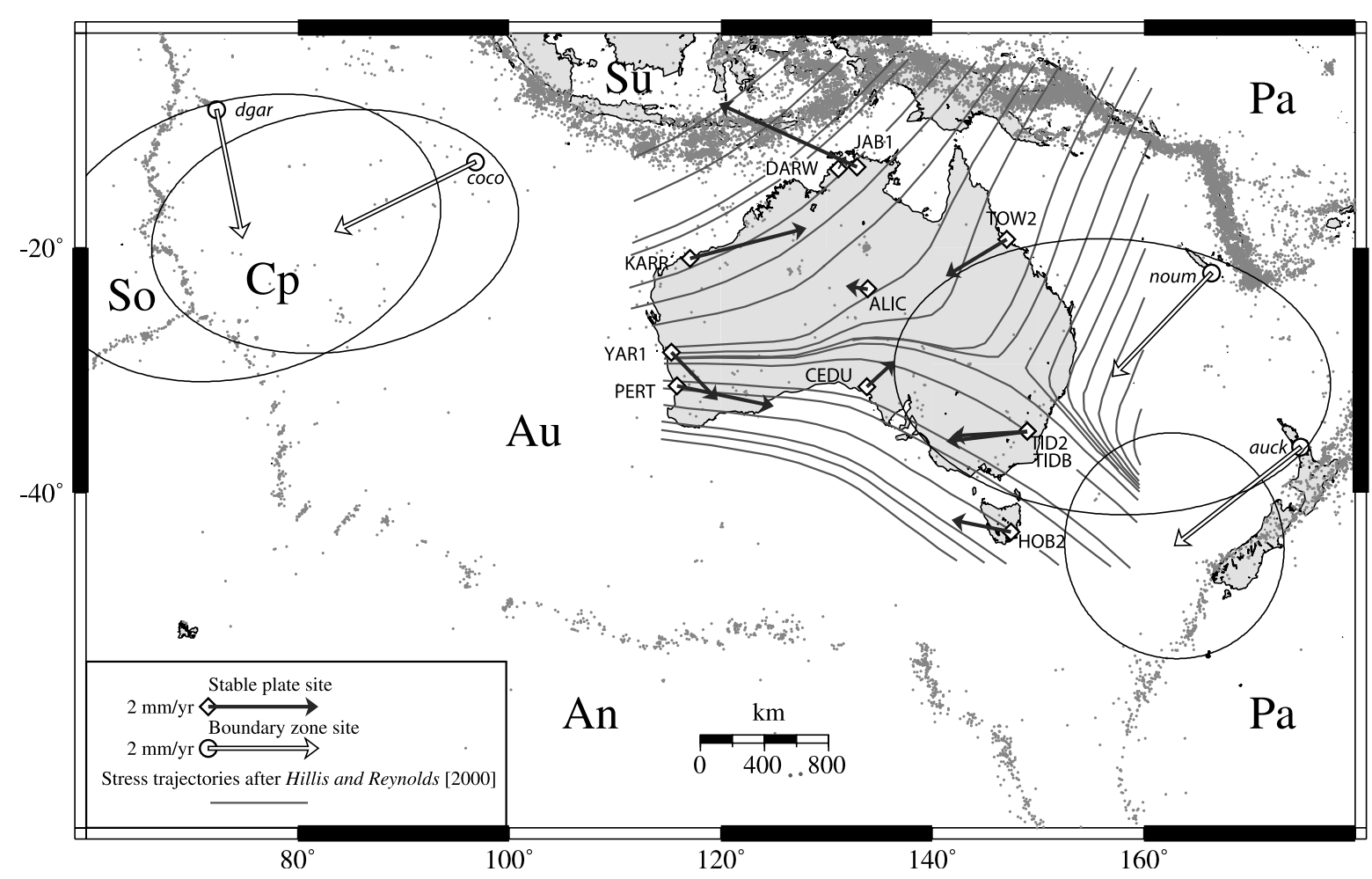

Figure 10. Lambert azimuthal equal-area projection showing space geodetic site locations for the Australian plate and earthquake epicenters (magnitude $>4.5$, depth $<50 \mathrm{~km}$, between 1973 and 2000), indicating plate boundaries. Sites with diamonds and upper case letters are assumed to lie on the stable plate interior and are used to define the plate's angular velocity. Their residual velocities with respect to the plate interior are shown by solid arrows (Table 2), and for clarity, their error ellipses are omitted. Sites with circles and lowercase letters lie in deforming boundary zones and are not used in the rigid plate definition. Their residual velocities with respect to the plate interior are shown with open arrows (Table 2) and error ellipses representing two-dimensional 95\% confidence regions. Stress trajectories (orientation of maximum horizontal compression) from Hillis and Reynolds [2000] are superimposed, showing a similar pattern to residual velocities in the plate interior. See Table 1 for plate abbreviations, except $\mathrm{Cp}$, Capricorn.

coco or dgar reflects the fact that these two sites have velocities that are negligibly different from stable Australia, consistent with very slow relative motion between Capricorn and Australia [Royer and Gordon, 1997; Conder and Forsyth, 2001].

4.3.5. Caribbean. [34] The Caribbean plate has been the focus of kinematic studies for at least 25 years [Jordan, 1975; Stein et al., 1988; Deng and Sykes, 1995; DeMets et al., 2000]. Geologic estimates of the velocity of the Caribbean plate with respect to its neighbors are hampered by the paucity of relevant data (especially rate data from spreading centers) and the tectonic complexity of the boundary zones around the plate (see discussions by DeMets et al. [1990] and Dixon et al. [1991b]). The velocity data set used here is similar to that presented by Weber et al. [2001], adding only additional position data at the continuous sites BARB and CRO1. Also, we take a slightly more conservative approach, omitting two sites in Puerto Rico (isab, pur3) because of the possibility of independent motion of the Puerto Rico block [Jansma et al., 2000]. The resulting Caribbean-South America angular velocity is nevertheless essentially identical to that presented by Weber et al. [2001] (Table 5). The Caribbean-North America angular velocity is similar to that presented by DeMets et al. [2000], although the current vector is constrained by additional position data at SANA, ROJO, and CRO1, which improves their velocity estimate, as well as by velocities from new sites at BARB and TDAD. As pointed out by Dixon et al. [1998], Pollitz and Dixon [1998], DeMets et al. [2000], Weber et al. [2001], and Perez et al. [2001], the motion of the Caribbean plate with respect to both North and South America is considerably faster than predicted by NUVEL-1A, probably reflecting systematic errors in the geologic model (Table 5).

[35] With the benefit of hindsight, it is clear why NUVEL-1A systematically underestimates the speed of the Caribbean plate (the predicted azimuths for NUVEL-1A and REVEL-2000 are very similar along much of the Caribbean boundary). Sykes et al. [1982], Rosencrantz and Mann [1991], and Mann et al. [1995] discuss the importance of the Gonave microplate, separating the North American and Caribbean plates. This microplate is defined by the Cayman spreading center on the west [Macdonald and Holcombe, 1978; Rosencrantz et al., 1988], the Oriente-Septentrional fault zone on the north, and the Enriquillo-Plantain Garden fault zone on the south [Leroy et al., 2000; Pubellier et al., 2000] (Figure 11). Dixon et al. [1998] estimated $8 \pm 4 \mathrm{~mm} / \mathrm{yr}$ of motion along the Enriquillo fault zone in the Dominican Republic, a significant fraction of overall North America-Caribbean motion. NUVEL-1A underestimates Caribbean-North America (and, by implication, Caribbean-South America) motion by roughly the slip rate on this fault because this rate represents that portion of plate motion not accommodated on the Cayman spreading center, NUVEL-1A's only Caribbean rate datum. REVEL-2000 provides a more accurate estimate of Caribbean-North America and Caribbean-South America motion, not only for the decade timescale but perhaps for the last few million years as well, because it represents the total relative plate motion, not just motion accommodated at the spreading center.

4.3.6. Eurasia. [36] We define the Eurasian plate using 15 sites (Figure 5 and Table 1) and obtain a well-defined 


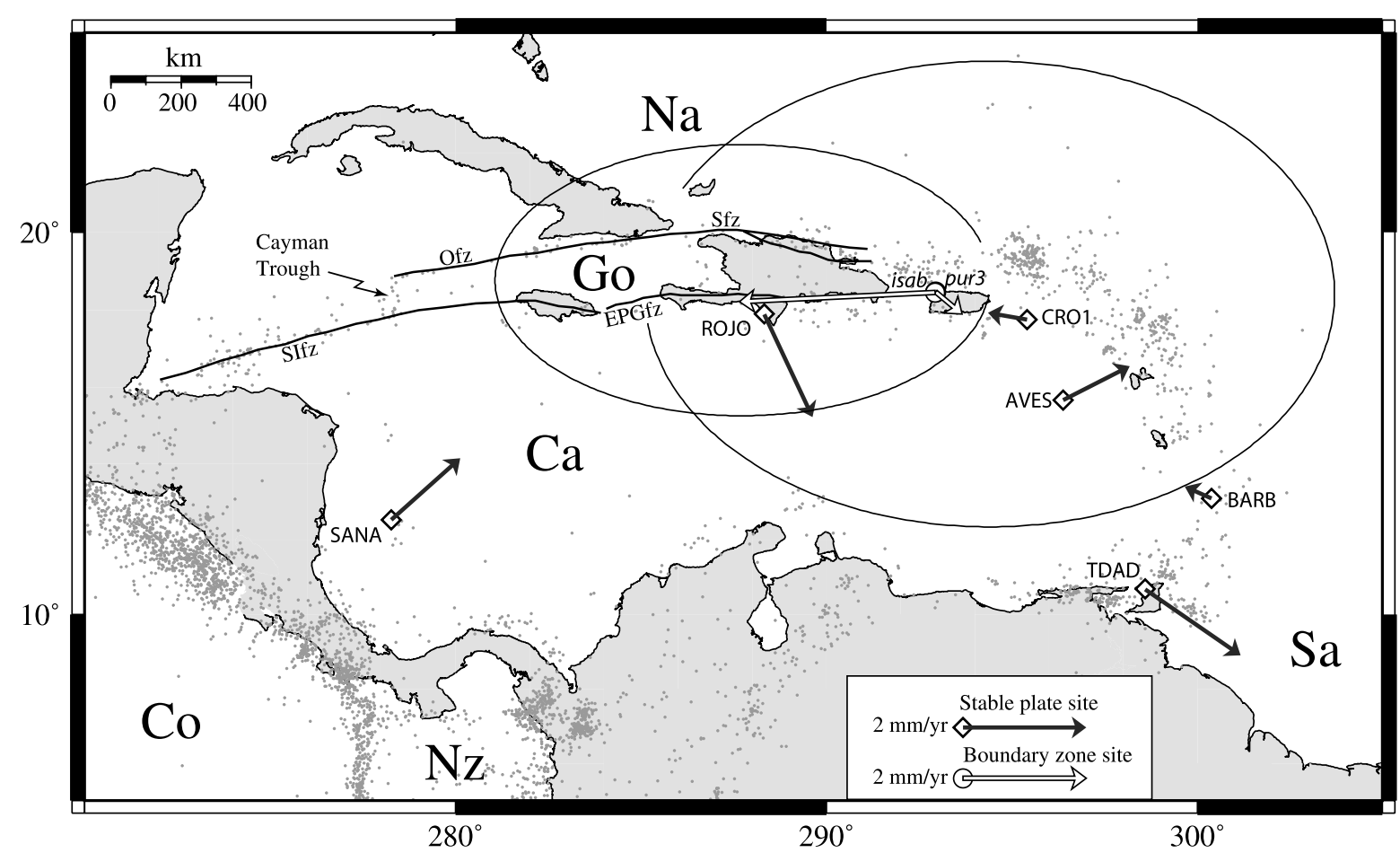

Figure 11. Similar to Figure 10, for the Caribbean plate. EPGfz, Enriquillo-Plantain Garden fault zone; Go, Gonave block; Ofz, Oriente fault zone; Sfz, Septentrional fault zone; SIfz, Swan Island fault zone.

solution $\left(\chi_{v}^{2}=1.02\right.$, mean rate residual of $\left.0.71 \mathrm{~mm} / \mathrm{yr}\right)$. We exclude all sites south of significant seismicity reflecting the Nubia-Eurasia collision (e.g., south of the Pyrenees we exclude casc, ebre, and vill [Ribeiro et al., 1996; Herraiz et al., 2000]), all sites in or south of the Alps and south of the Carpathians (geno, noto, penc, sofi) [Calais, 1999; Grenerczy et al., 2000], all sites in central Asia south of $45^{\circ} \mathrm{N}$ because they may be affected by the India-Eurasia collision (kit3, pol2, sumk, urum), and all sites east of $130^{\circ} \mathrm{W}$ that may be on the North American plate or in the deforming zone between North America and Eurasia (bili). We also exclude sites in or near the Rhine Graben and Roer Graben (dour, kosg, wsrt) and sites west of the Rhine Graben and south of the Roer Graben (brst, brus, hers, mans, mlvl, sjdv, toul) similar to Nocquet et al. [2001]. These grabens were active in late Pliocene time and are also the current locus of significant seismicity [Plenefisch and Bonjer, 1997]. In addition, irkt is excluded because it is $<100 \mathrm{~km}$ from the active Baikal Rift [Doser, 1991; Delvaux et al., 1997] (see section 4.3.1), and yakz is excluded because of problems with its time series that we do not understand (our time series for this site shows a large seasonal variation in all three components). We also omitted kiru, mets, onsa, and trom due to sensitivity to GIA, as discussed earlier. Site brst shows a large residual motion with respect to Eurasia, possibly reflecting neotectonic effects in the area [van Vliet-Lanoe et al., 1997]. A Eurasia solution that excludes TIXI, near the possible plate boundary with North America, results in an angular velocity that is negligibly different (14-site solution $58.37^{\circ} \mathrm{N},-102.06^{\circ} \mathrm{E}, 0.258^{\circ} / \mathrm{Myr}, \sigma_{\text {maj }}=1.7$, $\sigma_{\min }=0.4, \sigma_{\omega}=0.004^{\circ} / \mathrm{Myr}, \chi_{v}^{2}=1.10, \mathrm{MRR}=0.7 \mathrm{~mm} / \mathrm{yr}$ ).

[37] Inclusion of the available six sites in stable western Europe (west of the Rhine Graben) in the Eurasia plate solution increases misfit $\left(\chi_{v}^{2}=1.70\right.$ for 21 sites compared to our preferred 15-site solution with $\left.\chi_{v}^{2}=1.02\right)$, but the difference is small enough that separate western Europe and Eurasian plates are not supported by an $F$ ratio test [Stein and Gordon, 1984] (angular velocity for the six sites west of the Rhine Graben with respect to ITRF-97 is $45.64^{\circ} \mathrm{N},-117.08^{\circ} \mathrm{E}, 0.215^{\circ} / \mathrm{Myr}, \sigma_{\text {maj }}=18.1, \sigma_{\min }=0.9$, $\sigma_{\omega}=0.016^{\circ} / \mathrm{Myr}, \chi_{v}^{2}=1.46, \mathrm{MRR}=0.8 \mathrm{~mm} / \mathrm{yr}$ ). The two plates may exist, but our data are insufficient to reliably resolve their relative motion. Given the observed seismicity and surface faulting in the Rhine Graben [Camelbeeck and Meghraoui, 1998; Meghraoui et al., 2000], we have taken the more conservative approach and used only sites east of the rift in our definition of Eurasia.

[38] We can nevertheless calculate relative motion across the Rhine Graben, using the admittedly noisy relative angular velocity vector between Asia and western Europe as defined above $\left(58.48^{\circ} \mathrm{N}, 2.35^{\circ} \mathrm{E}, 0.076^{\circ} / \mathrm{Myr}, \sigma_{\text {maj }}=9.4, \sigma_{\text {min }}=2.7\right.$, $\sigma_{\omega}=0.005^{\circ} / \mathrm{Myr}$ ). Calculated at a point on the upper Rhine Graben $\left(49^{\circ} \mathrm{N}, 8^{\circ} \mathrm{E}\right)$ we predict west-southwest/east-northeast extension, $1.5 \pm 0.4 \mathrm{~mm} / \mathrm{yr}$ at $73 \pm 17^{\circ}$. Note that this calculation does not require that relative motion between western Europe and Eurasia is accommodated exclusively in the Rhine Graben. Our calculated direction agrees with the orientation of minimum principal stress from earthquake focal mechanisms in the region [Plenefisch and Bonjer, 1997] and is consistent with Nocquet et al. [2001]. Westward motion of western Europe may reflect extrusion tectonics associated with the northward motion of Italy and the Nubian plate relative to Eurasia.

[39] Following Kogan et al. [2000], we evaluate if site bili in northeastern Russia lies on the North American or Eurasian plate by comparing the size of the corresponding velocity residual. This site has a residual rate of $6.4 \pm 2.0 \mathrm{~mm} / \mathrm{yr}$ with respect to Eurasia and $3.4 \pm 1.6 \mathrm{~mm} / \mathrm{yr}$ with respect to North America, suggesting that bili lies either on the North American plate or on the diffuse boundary between the two plates [Chapman and Solomon, 1976; Zonenshain and Savostin, 1981; Cook et al., 1986].

[40] We can also compare the predictions of our angular velocity estimate for Eurasia-North America with measured spreading rates and transform fault azimuths along the Mid-Atlantic Ridge and with the NUVEL-1A model. Figure 12 shows this comparison and also the predictions of Kogan et al. [2000]. REVEL-2000 shows good agreement with the geologic data along the Mid-Atlantic Ridge north of about $65^{\circ} \mathrm{N}$ but is systematically faster than a cluster of geologic data around $40^{\circ}-45^{\circ} \mathrm{N}$ by $\sim 2 \mathrm{~mm} / \mathrm{yr}$. At $64^{\circ} \mathrm{N}$, $20.5^{\circ} \mathrm{W}$ on the plate boundary in Iceland we predict spreading 

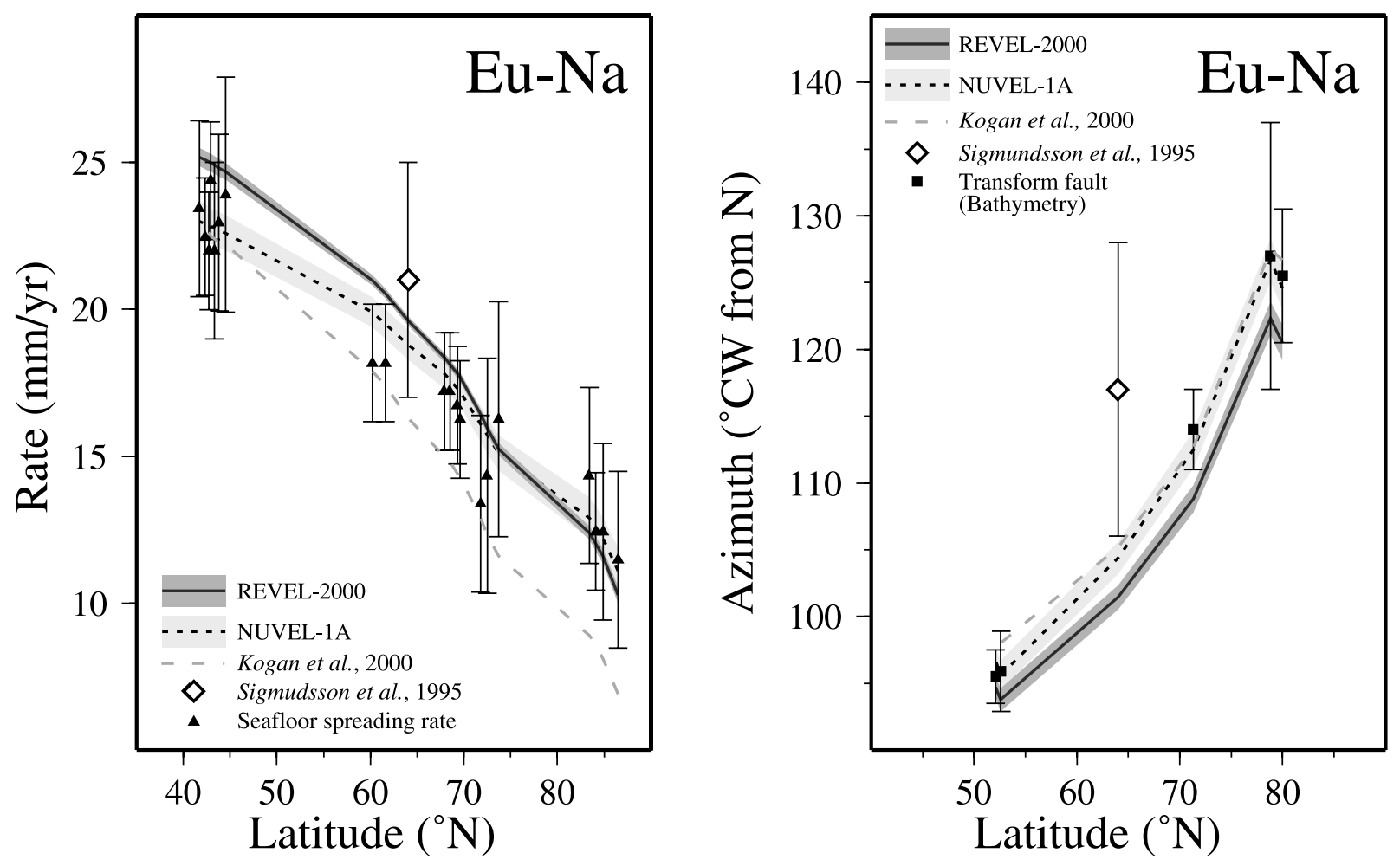

Figure 12. Similar to Figure 6, for Eurasia-North America.

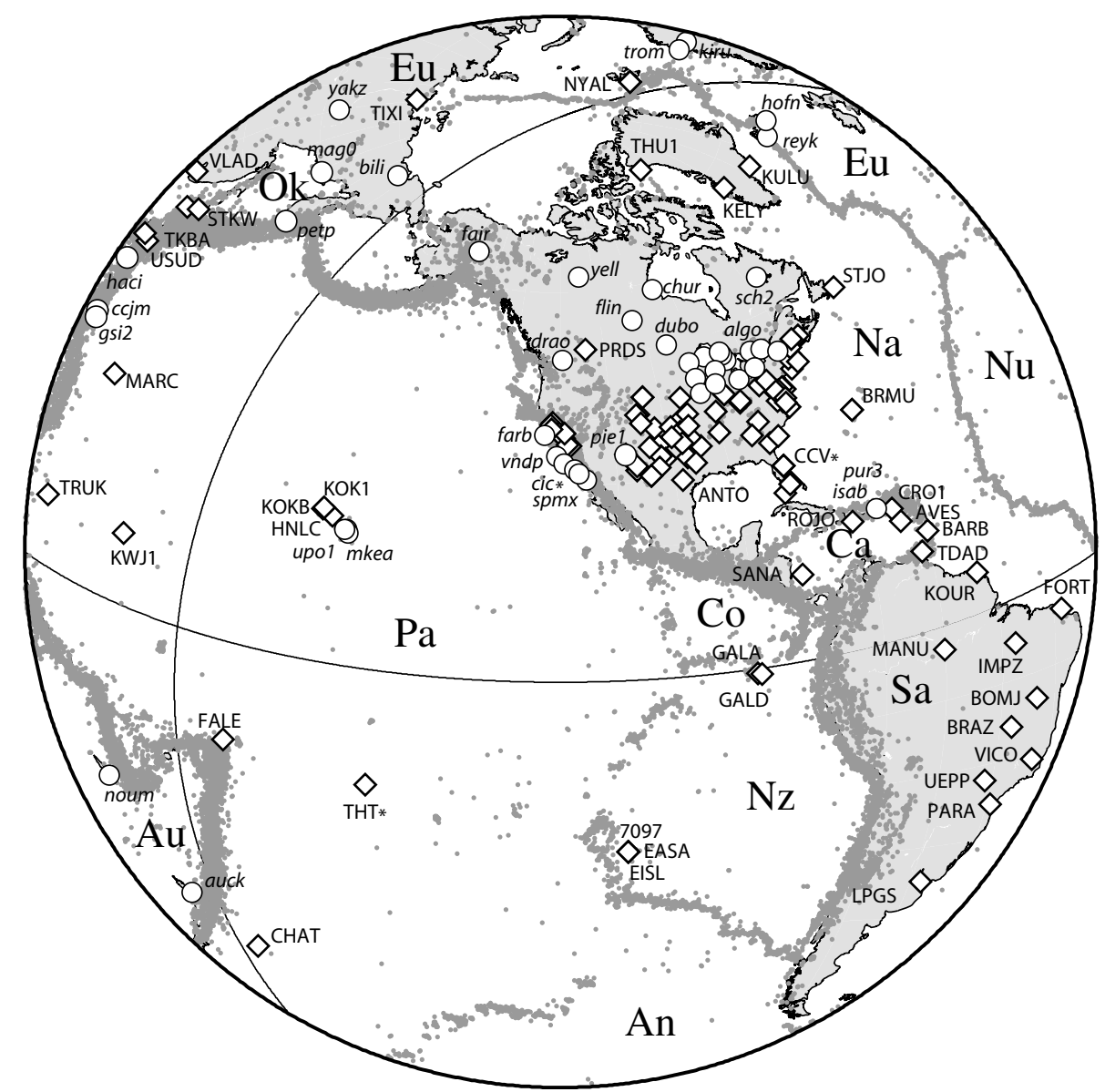

Figure 13. Similar to Figure 5 for the Pacific Ocean area. For clarity, sites on North America are not labeled. Co, Cocos plate. 

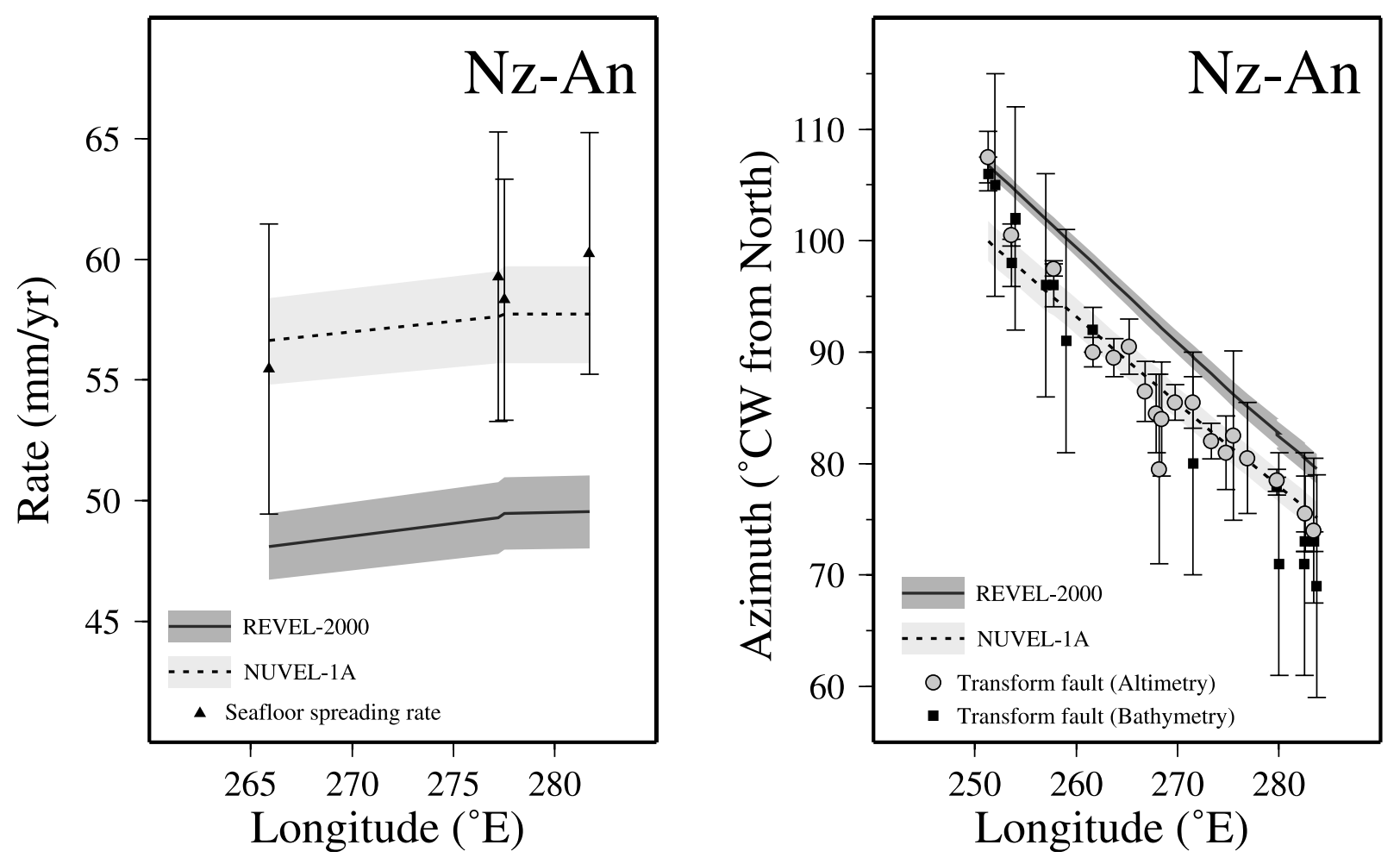

Figure 14. Similar to Figure 6, for Nazca-Antarctica.

at a rate of $19.9 \pm 0.2 \mathrm{~mm} / \mathrm{yr}$ at an azimuth of $102 \pm 1^{\circ}$, which compares well with the local measured velocity using episodic GPS data, $21 \pm 4 \mathrm{~mm} / \mathrm{yr}$ at azimuth of $117 \pm 11^{\circ}$ [Sigmundsson et al., 1995].

[41] We can determine what fraction of the total plate motion is accommodated within Iceland by comparing the velocity of hofn in the southeastern corner of the island relative to stable North America $\left(21.2 \pm 0.8 \mathrm{~mm} / \mathrm{yr}\right.$ at $\left.104.1 \pm 2.0^{\circ}\right)$ with the relative plate velocity computed at the same location $\left(19.7 \pm 0.2 \mathrm{~mm} / \mathrm{yr}\right.$ at $\left.104.2 \pm 0.9^{\circ}\right)$ (hofn is not used in the definition of Eurasia, so this is an independent test). Thus hofn's velocity is consistent with stable Eurasia's velocity within $95 \%$ confidence. Similarly reyk in southwestern Iceland is not used in the definition of stable North America and moves at $20.2 \pm 0.6 \mathrm{~mm} / \mathrm{yr}$ at $281.4 \pm 1.8^{\circ}$ relative to stable Eurasia, compared to the calculated relative plate velocity here of $19.9 \pm 0.2 \mathrm{~mm} / \mathrm{yr}$ at $280.8 \pm 0.9^{\circ}$, again equivalent within uncertainties. The baseline between hofn and reyk is more precise than the site velocities described above and is independent of any definition of stable North America or Eurasia. Its length rate of change, $20.3 \pm$ $0.2 \mathrm{~mm} / \mathrm{yr}$, is very similar to the predicted rate of change from REVEL-2000, $19.8 \pm 0.2 \mathrm{~mm} / \mathrm{yr}$, calculated at the point where the baseline between these two sites crosses the plate boundary $\left(64.2^{\circ} \mathrm{N}\right.$, $\left.18.8^{\circ} \mathrm{W}\right)$. These results confirm that essentially all of the plate motion between North America and Eurasia is accommodated within the island, consistent with earlier studies [Sigmundsson et al., 1995; Jonsson et al., 1997; Hreinsdottir et al., 2001].

4.3.7. India. [42] We define the Indian plate using three sites, HYDE, IISC, and MALD. Our mean rate residual $(1 \mathrm{~mm} / \mathrm{yr})$ is compatible with earlier rigidity studies of Paul et al. [1995] and Malaimani et al. [2000]. We agree with earlier findings suggesting that the Indian plate is moving slower than predicted by NUVEL-1A [Chen et al., 2000; Shen et al., 2000; Holt et al., 2000; Paul et al., 2001; Kreemer et al., 2000]. These studies show a range of velocity estimates (e.g., rates at IISC relative to Eurasia of 34.8, 41.9, 36, 43.7 , and $34.3 \mathrm{~mm} / \mathrm{yr}$, respectively), but all are slower than the corresponding NUVEL-1A estimate $(47.8 \mathrm{~mm} / \mathrm{yr})$. Our EurasiaIndia angular velocity predicts a rate of $35.2 \mathrm{~mm} / \mathrm{yr}$ at this location. It is unclear whether the difference between the geodetic and geologic rates reflects a true deceleration in the relative velocity of this plate pair or a systematic error in NUVEL-1A [Gordon et al., 1999].

4.3.8. Nazca and South America. [43] Owing to the paucity of GPS data on the Nazca plate (EISL, GALA) we include all available space geodetic data, adding SLR (7097) and DORIS (EASA, GALD) (Figure 13). Previous estimates for South America were also hampered by relatively sparse data. For example, Norabuena et al. [1998] had only four continuous GPS sites with a total of $\sim 3000$ station days of data to define stable South America, while Norabuena et al. [1999] had 6200 station days of data, also at four sites. For this study we have 11 GPS sites with 14,200 station days of data; hence South America's velocity is much better defined. Our resulting Nazca-South America rate is nevertheless consistent with previous results [Norabuena et al., 1998, 1999; Angermann et al., 1999] and significantly slower than the NUVEL-1A estimate. Nazca-Antarctica and Nazca-Pacific are also significantly slower than the geologic model (Figures 14 and 15). As has been shown in previous studies, both NazcaPacific and Nazca-South America have been decelerating during the last $25 \mathrm{Myr}$ [Tebbens and Cande, 1997; Somoza, 1998]. A similar deceleration applies to Nazca-Antarctica (Figure 16). These decelerations are sufficiently rapid that they can be observed as differences between geodetic plate motion estimates and NUVEL$1 \mathrm{~A}$ predictions.

[44] The REVEL-2000 velocity azimuths for Nazca-Antarctica and Nazca-Pacific also differ from NUVEL-1A (Figure 16). Although the Nazca site distribution is limited, the difference may be real, perhaps reflecting changing plate direction over time. Stage pole reconstructions back to $30 \mathrm{Ma}$ [Tebbens and Cande, 1997] allow us to look at a much longer time record and suggest changes in Nazca-Antarctica and Nazca-Pacific direction in the last 15 Myr in the same sense as we infer from the shorter epochs "sampled" by NUVEL-1A and REVEL-2000 (compare Figures 14 and 16).

4.3.9. North America. [45] The rigid North American plate is defined using 64 sites (Figure 13 and Table 1). We exclude all sites located in the Gulf Coast because of possible subsidence and 

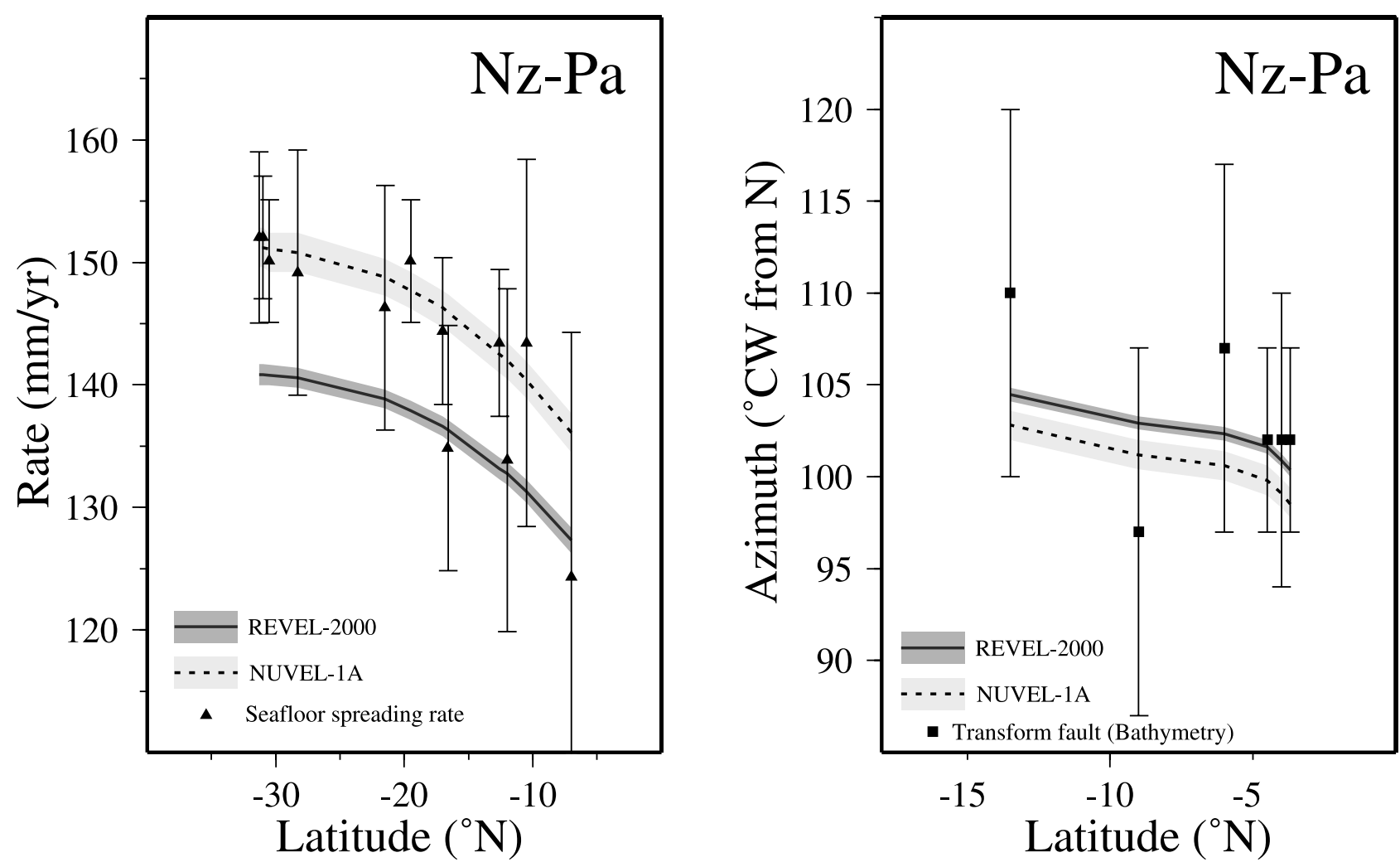

Figure 15. Similar to Figure 6, for Nazca-Pacific.

all sites west of the Rio Grande Rift and west of the Rocky Mountain front because of possible tectonic effects. Sites mem2 in Memphis, Tennessee, and cha1 in Charleston, South Carolina, are also omitted as they have both been the focus of major intraplate earthquakes in the last 200 years [Nuttli, 1973; Bollinger, 1972]. As discussed earlier, we omit 20 sites likely to be most affected by glacial isostatic adjustment in the northern part of the plate. A more detailed discussion of North America is in preparation.
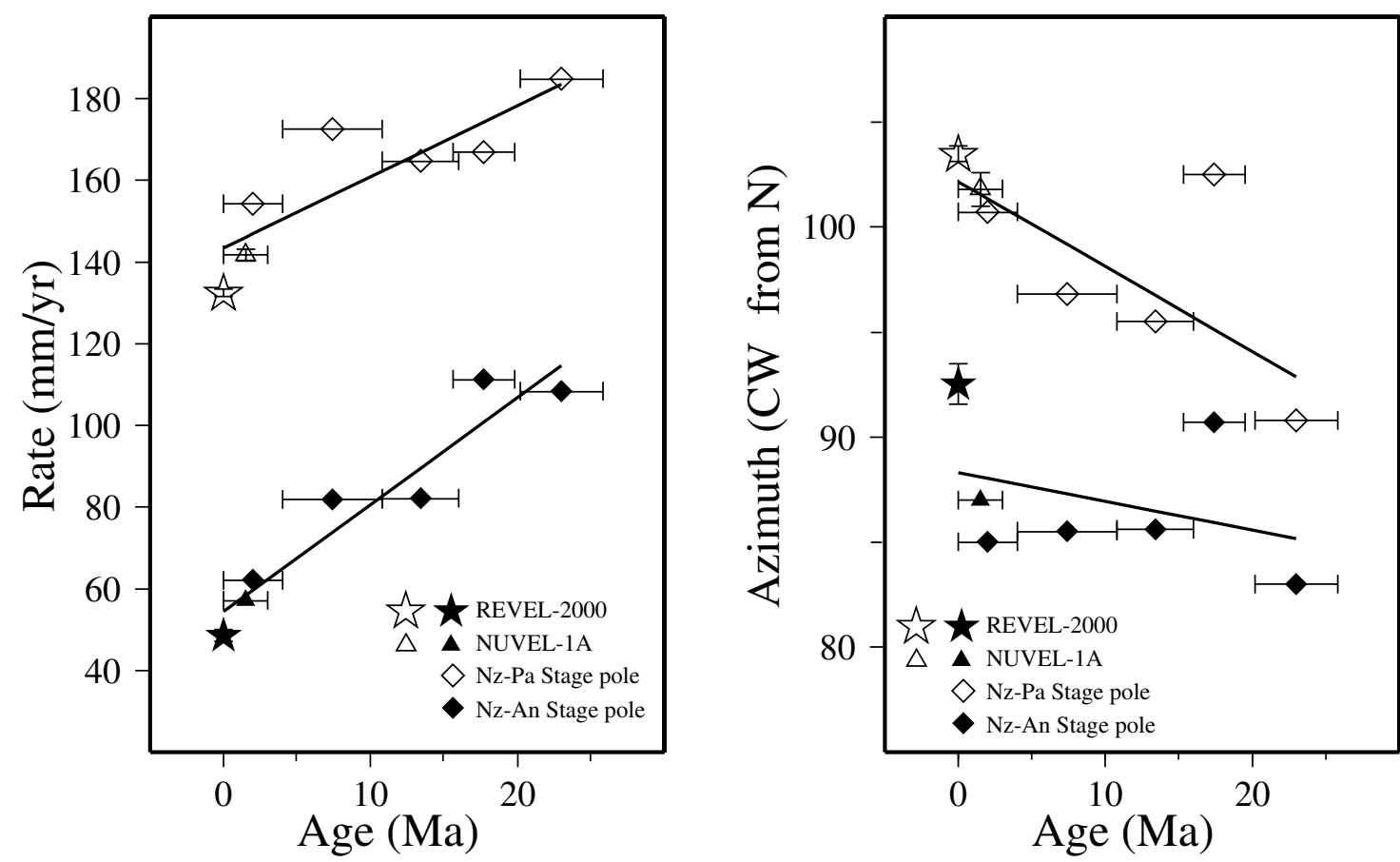

Figure 16. Spreading rates and transform azimuths predicted for Nazca-Pacific (calculated at $12^{\circ} \mathrm{S}, 250^{\circ} \mathrm{E}$ ) and Nazca-Antarctic (calculated at $40^{\circ} \mathrm{S}, 268^{\circ} \mathrm{E}$ ) plates using NUVEL-1A, REVEL-2000, and stage pole data of Tebbens and Cande [1997]. Note more rapid deceleration implied by post-10 Ma data. 


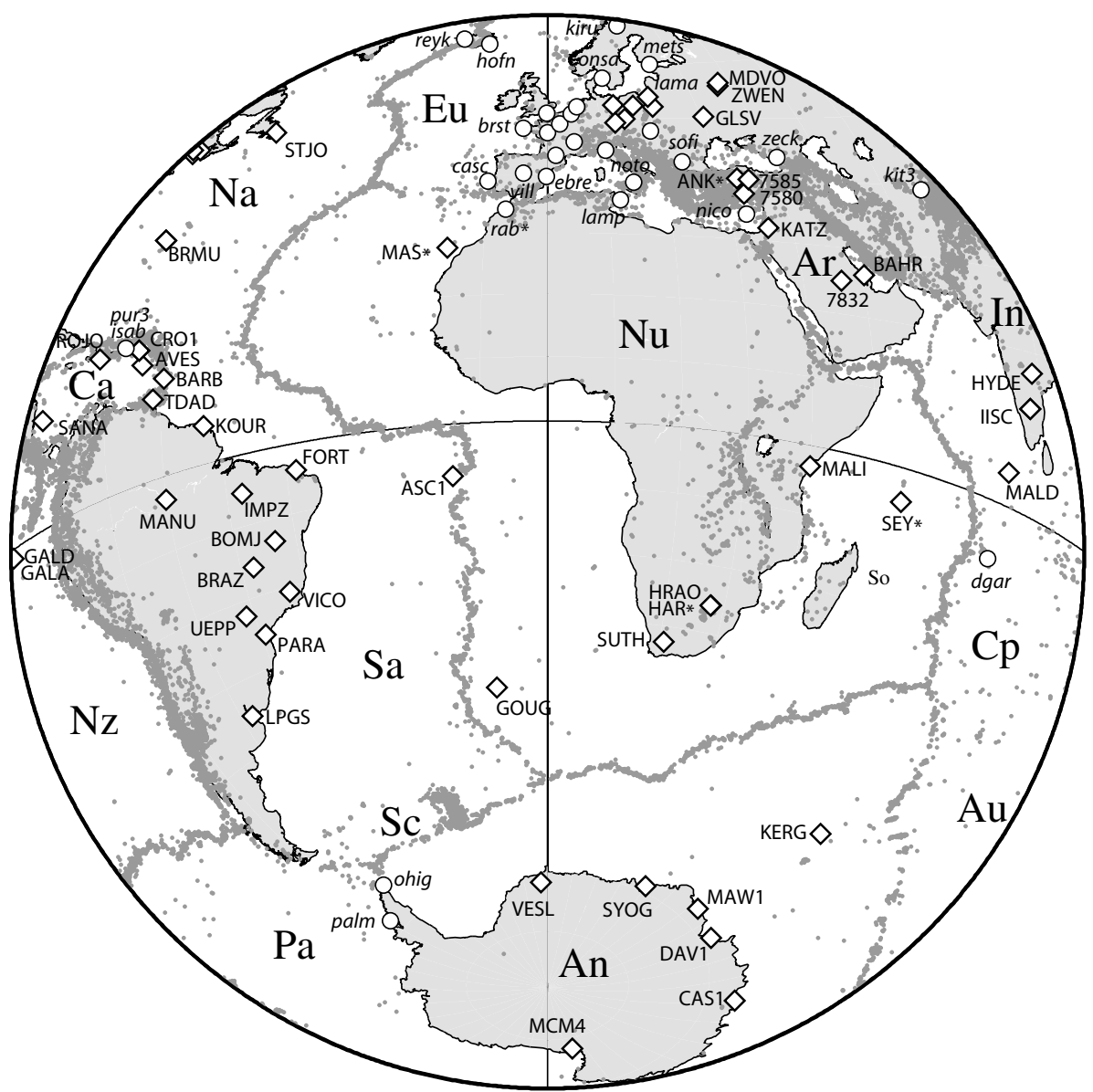

Figure 17. Similar to Figure 5 for the Atlantic Ocean area. Sc, Scotia plate.
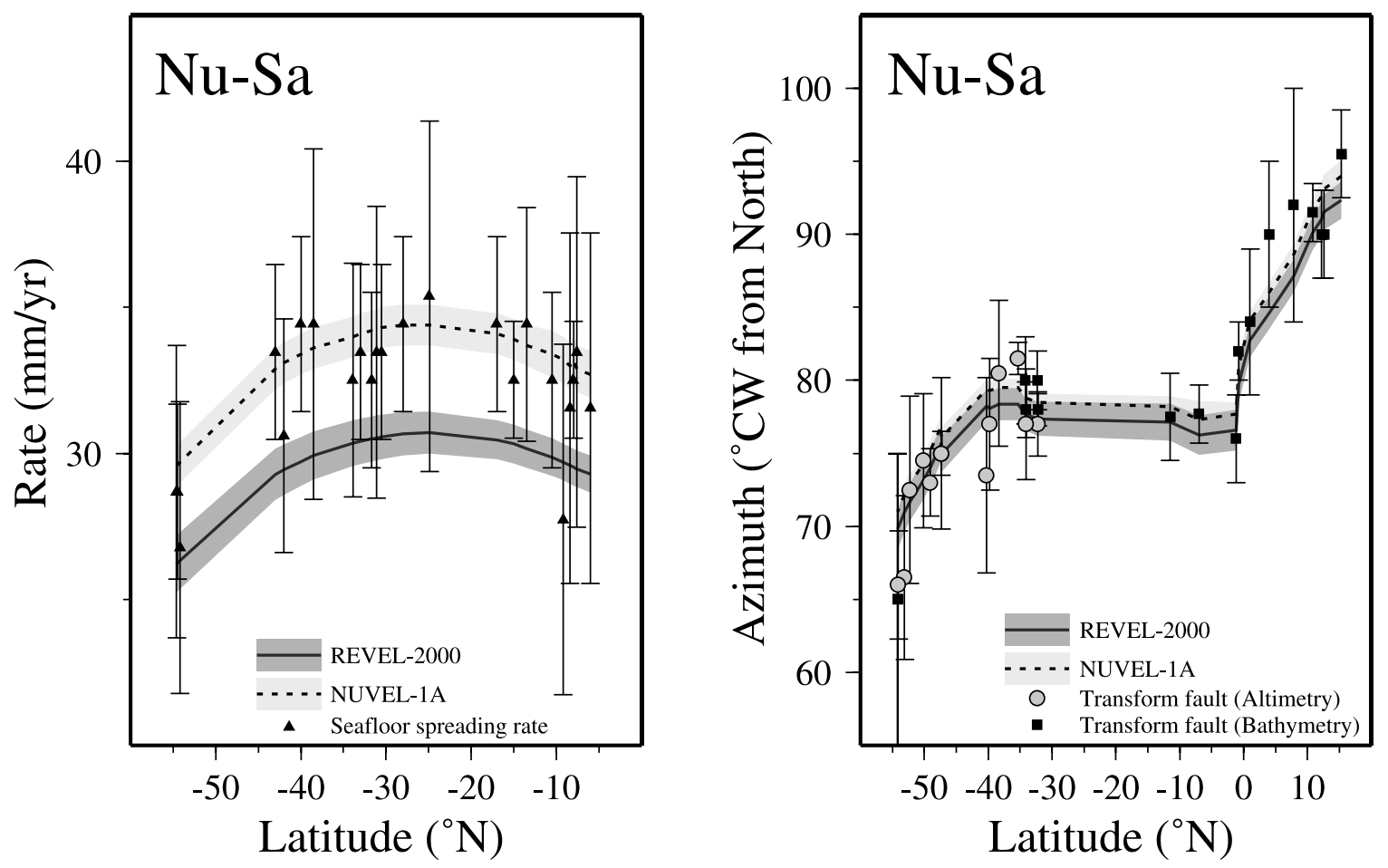

Figure 18. Similar to Figure 6, for Nubia-South America. 


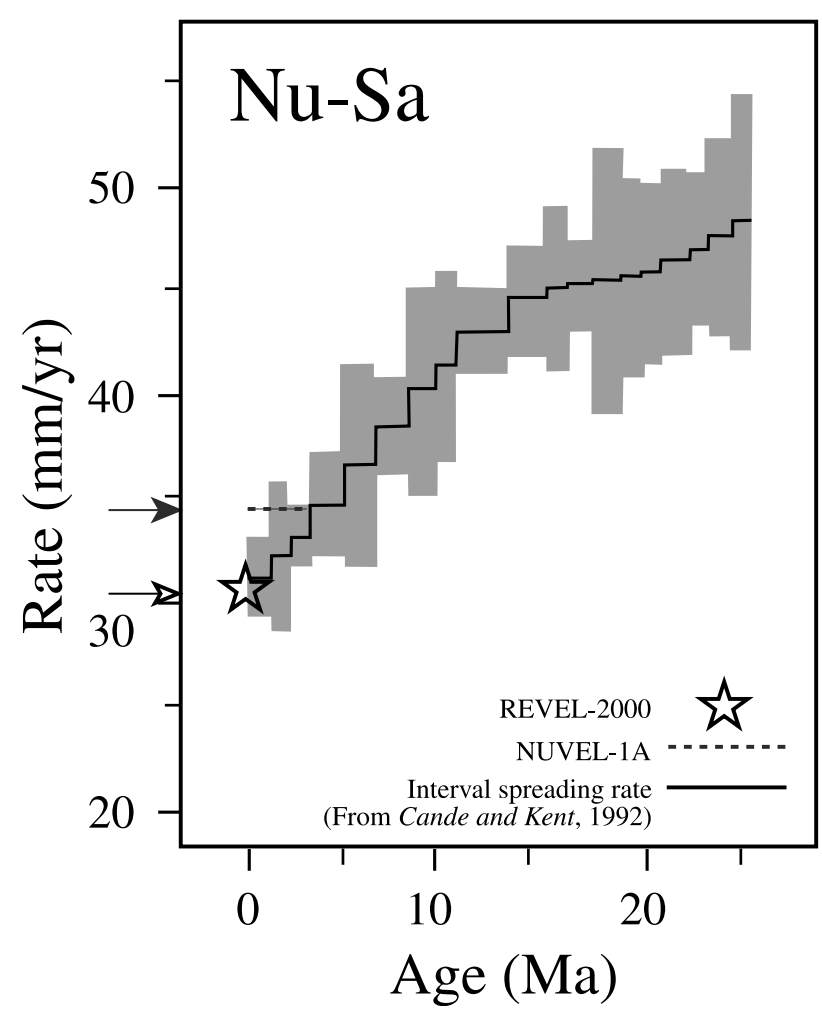

Figure 19. Nubia-South America spreading rates (full rate) versus age for last $25 \mathrm{Myr}$, from Cande and Kent [1992] (shaded area shows $\sim 1$ standard error), compared to NUVEL-1A and REVEL-2000 predictions (arrows) calculated at $28^{\circ} \mathrm{S}, 348^{\circ} \mathrm{E}$. REVEL-2000 differs significantly from NUVEL-1A but agrees closely with an extrapolation of interval spreading rates to Recent time.

[46] Fairbanks, Alaska (fair), has been used in our previous definitions of stable North America [e.g., Dixon et al., 1996]. It is excluded here because it is too close to the seismically active plate boundary zone and moves $2.2 \pm 0.5 \mathrm{~mm} / \mathrm{yr}$ at $171 \pm 11.4^{\circ}$ relative to stable North America (Table 1), somewhat faster than would be expected for a "stable plate" interior site.

4.3.10. Nubia. [47] We define an angular velocity for Nubia using five sites (GOUG, HAR*, HRAO, MAS*, SUTH). HRAO and HAR* in South Africa are located near an area of intense microseismicity (Figure 17). A three-site solution that omits these two sites has an angular velocity of $52.65^{\circ} \mathrm{N},-82.79^{\circ} \mathrm{E}, 0.249^{\circ}$ / Myr, $\sigma_{\max }=1.9, \sigma_{\min }=0.6, \sigma_{\omega}=0.004, \chi_{v}^{2}=0.27, \mathrm{MRR}=0.79$ $\mathrm{mm} / \mathrm{yr}$, very similar to our preferred five-site solution (Table 2), and suggests that sites HRAO and HAR* can be included in the stable plate solution.

[48] Our predicted Nubia-South America velocity should not be directly comparable to the NUVEL-1A model, since NUVEL-1A is based on a composite African plate, while we define separate Nubia and Somalia plates. However, we can still compare with the underlying geologic data (Table 5). Despite this difference, REVEL-2000 and NUVEL-1A azimuths in the South Atlantic are indistinguishable from each other and from the geologic data (Figure 18). However, the REVEL-2000 and NUVEL-1A rates differ significantly, with REVEL-2000 slower than both NUVEL-1A and the geologic data upon which it is based, by $\sim 4 \mathrm{~mm} / \mathrm{yr}$ through a large range of latitudes (Figure 18). The simplest interpretation is that this reflects true deceleration rather than a systematic bias in either model, since both models are well constrained in this region. Longer-term geologic data support this inference. Figure 19, modified from Cande and Kent [1992], shows a remarkable agreement between the REVEL-2000 geodetic rate and a longerterm trend of decelerating spreading in the south Atlantic going back $\sim 25$ Myr. This is roughly the time of initiation of the current phase of Andean crustal shortening (see discussion of slowing Nazca-South America convergence by Norabuena et al. [1999, and references therein]). It is tempting to speculate that the same process that has slowed convergence between South America and Nazca (e.g., formation of the Andes, associated crustal thickening, and possible increased resistance to subduction) may also contribute to a gradual slowing of South America's westward component of motion and consequent slowing of spreading in the south Atlantic. For example, growth of the Andes and high topography would increase South America's east directed gravitational body force, opposing the west directed body force associated with spreading at the Mid-Atlantic Ridge.

4.3.11. Pacific. [49] We define the Pacific plate using 9 sites (Table 1 and Figure 13), excluding two sites (mkea and upo1) on the Big Island of Hawaii that may be affected by deformation associated with the active Mauna Loa and Kilauea volcanoes. We agree with the conclusions of DeMets and Dixon [1999] that the NUVEL-1A angular velocity for Pacific-North America significantly underestimates present-day Pacific-North American motion. DeMets [1995] discussed possible biases in the NUVEL1A estimate for Pacific-North America, in particular, the rate data based on magnetic anomalies from the Alarcon Rise in the southern Gulf of California, which do not reflect the full plate motion due to faults to the west that have been active for most of the last 3 Myr. These may also be active today [Dixon et al., 2000b]. Thus the bias in NUVEL-1A's estimate of Pacific-North America motion has essentially the same explanation as biases in Caribbean-North America and Caribbean-South America motion, namely, incorporation of rate data in the geologic model that, because of tectonic complexity, do not reflect total plate motion.

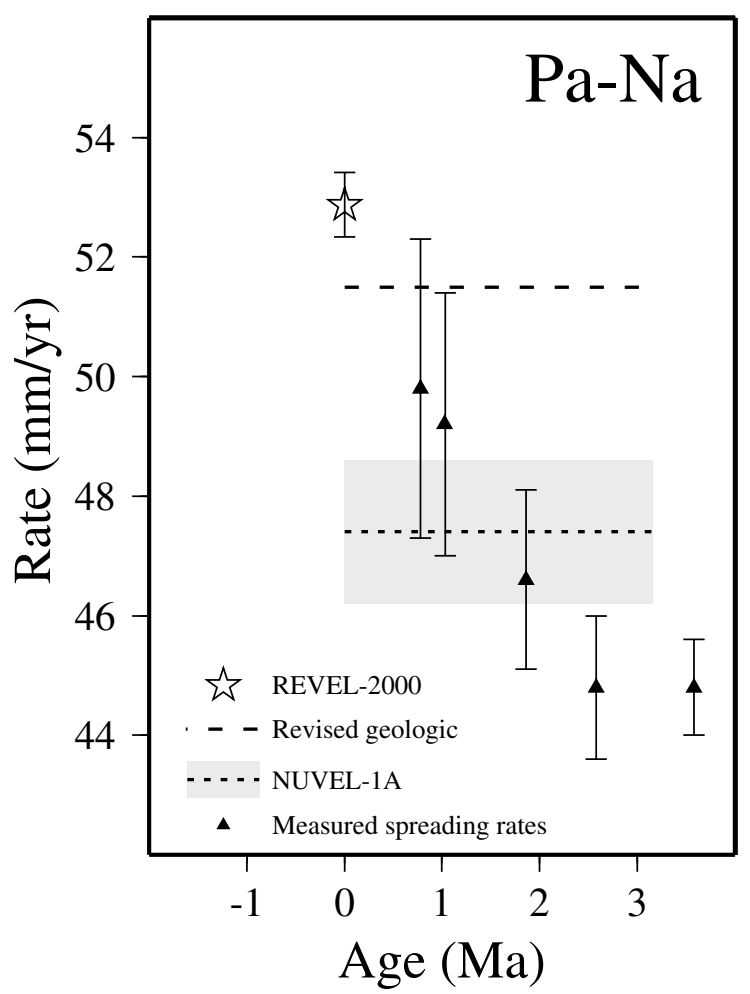

Figure 20. Measured seafloor spreading rate at various times in the southern Gulf of California corrected for outward displacement, from DeMets [1995], and predicted total motion from REVEL2000 calculated at $23.5^{\circ} \mathrm{N}, 251.5^{\circ} \mathrm{E}$. Revised geologic model from DeMets and Dixon [1999]. 


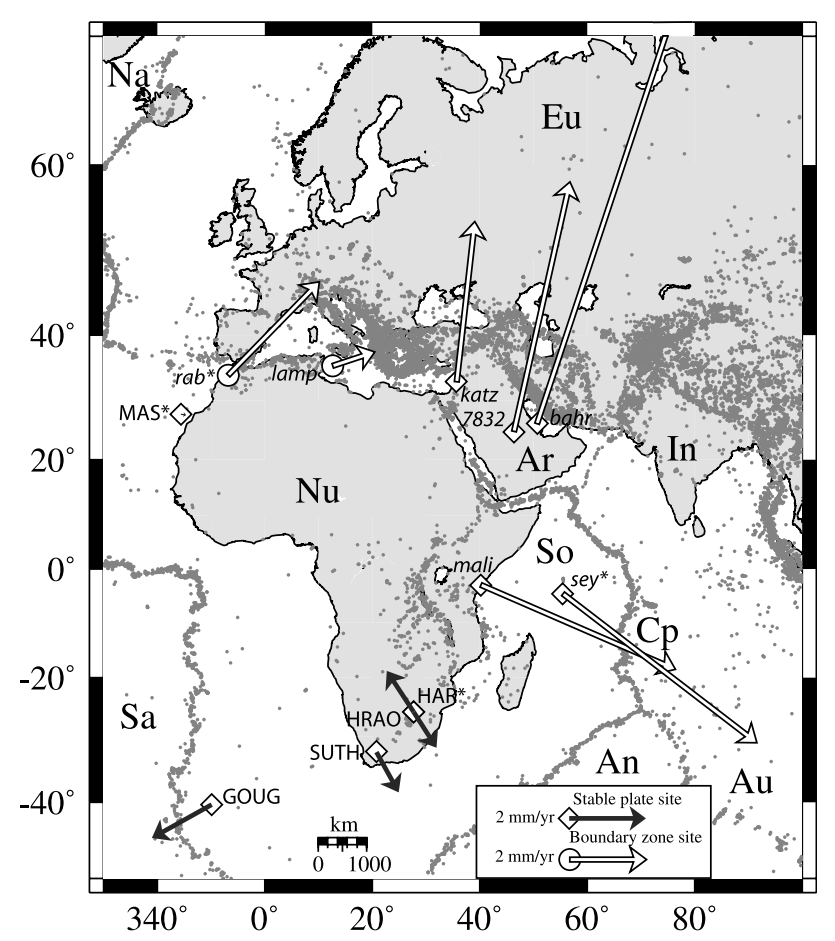

Figure 21. Similar to Figure 10, for the Nubian plate. Also shown are the velocity vectors for two sites on the Somalia plate (mali, sey*) and three sites on the Arabian plate (bahr, katz, 7832) relative to stable Nubia, indicating extension across the East African Rift, Red Sea, and Gulf of Aden. For clarity, error ellipses are omitted.

[50] Spreading in the southern Gulf of California shows a trend of increasing rate since $2.5 \mathrm{Ma}$ [DeMets, 1995], reflecting a combination of increased "focusing" of plate motion as spreading at the Alarcon Rise increasingly reflects the full plate motion and perhaps a small amount of acceleration. REVEL-2000's estimate of Pacific-North America motion is $1.4 \pm 0.5 \mathrm{~mm} / \mathrm{yr}$ faster than the geologic estimate of DeMets and Dixon [1999] for the southern Gulf of California (Figure 20).

[51] Six Pacific sites near the plate boundary zone with North America (cicz, farb, scip, sni1, spmx, vndp) have velocities slower than expected if they were on the rigid Pacific plate. Dixon et al. [2000b] and Beavan et al. [2002] explain these discrepancies by a combination of strain accumulation on locked faults of the San Andreas system, plus slip on additional faults offshore to the west [e.g., Sorlien et al., 1999]. Figure 20 suggests that such faults in the region of southern Baja California have a total slip rate less than $\sim 2 \mathrm{~mm} / \mathrm{yr}$ (the difference between the REVEL-2000 rate and estimated present-day spreading rate in the Alarcon Rise based on a linear fit to the magnetic anomaly rate data younger than $3 \mathrm{Ma})$.

[52] We are unable to directly verify our Australia-Pacific angular velocity against independent geologic data owing to the lack of transform faults or spreading ridges. Australia-Pacific stage pole data suggest little or no change over the last 11 Myr [Sutherland, 1995]. At a point on the Alpine fault in New Zealand $\left(43.5^{\circ} \mathrm{S}, 170^{\circ} \mathrm{E}\right), \mathrm{REVEL}-2000$ predicts $42.0 \pm 0.6 \mathrm{~mm} / \mathrm{yr}$ at an azimuth of $248 \pm 1^{\circ}$, giving $41 \pm 1 \mathrm{~mm} / \mathrm{yr}$ of fault-parallel slip (azimuth $236^{\circ}$ ) and $8 \pm 1 \mathrm{~mm} / \mathrm{yr}$ of shortening. The fault-parallel component is larger than the rate measured with episodic GPS, although the fault-normal component is comparable: Beavan et al. [1999] obtain $29.7 \pm 1.4 \mathrm{~mm} / \mathrm{yr}$ of fault-parallel slip and $9.9 \pm 1.8$ $\mathrm{mm} / \mathrm{yr}$ of shortening. Beavan et al. [1999] and Sutherland et al.
[2000] conclude that the Alpine fault and subparallel faults in New Zealand take up only $\sim 70-80 \%$ of total plate motion.

4.3.12. Philippine. [53] Angular velocity estimates for the Philippine plate by conventional geologic approaches are problematic because no spreading ridges bound the plate, and therefore geologic rate data are not available. This problem has been attacked with earthquake slip vector data [Seno et al., 1993] and GPS data [Kato et al., 1996, 1998; Kotake et al., 1998]. We use four sites (GSI1, GSI3, OKTO, and PAL*; $\chi_{v}^{2}=1.03$ ) to define the rigid Philippine plate (Figure 5). To test if sites close to the subducting Pacific plate experience strain accumulation or other nonrigid plate effects, we calculated residual velocities for ccjm, gsi2, and haci with respect to the rigid Philippine plate as defined above. Sites ccjm and gsi2, both $\sim 100 \mathrm{~km}$ from the trench, have residual velocities of $5.0 \pm 1.7 \mathrm{~mm} / \mathrm{yr}$ at $274 \pm 16^{\circ}$ and $3.0 \pm 1.8 \mathrm{~mm} / \mathrm{yr}$ at $142 \pm 32^{\circ}$, respectively. Site haci, $200 \mathrm{~km}$ from the trench, but within $100 \mathrm{~km}$ of Amuria (mainland Japan), has a residual of $11.1 \pm 1.7 \mathrm{~mm} / \mathrm{yr}$ at $119.9 \pm 5.4^{\circ}$. The eastward direction of the residuals at gsi2 and haci suggests that these sites are not strongly influenced by subduction strain accumulation from a seismically coupled Pacific plate (otherwise they would move west). Their eastward velocities may represent postseismic effects, or more likely slow spreading of a back arc basin behind the subduction zone. We cannot exclude the possibility that both effects occur, are of opposite sign, and sum to give the observed residual velocity. Site ccjm's westward residual velocity may reflect seismic coupling. Inclusion of any of these sites increases misfit $\left(4+\operatorname{ccjm}, \chi_{v}^{2}=2.05 ; 4+\right.$ gsi2, $\chi_{v}^{2}=1.19$; $4+$ haci, $\left.\chi_{v}^{2}=7.01\right)$. Site s102, located justoffshore Taiwan, clearly does not represent the stable interior of the Philippine plate $\left(4+\mathrm{s} 102, \chi_{v}^{2}=21.5\right)$ in agreement with $Y u$ et al. [1997, 1999].

[54] The velocity of guam with respect to the Philippine plate is a measure of spreading across the Mariana Trough, a back arc basin known to be actively extending. We predict a spreading velocity across the Mariana Trough at guam $\left(13.6^{\circ} \mathrm{N}, 144.9^{\circ} \mathrm{E}\right)$ of $46.6 \pm 1.5 \mathrm{~mm} / \mathrm{yr}$ at an azimuth of $096.3 \pm 1.8^{\circ}$, in broad agreement with published geologic rates (full spreading rate), 30-60 mm/yr [Bibee et al., 1980; Hussong and Uyeda, 1981; Ishihara et al., 2001]. Part of this range may reflect a change in spreading rate with latitude, with rate increasing to the south [Stern et al., 1984]. The subduction velocity of the Pacific plate relative to the overlying plate at the southern Mariana trench is defined by guam's velocity relative to the Pacific, $63.4 \pm 1.1 \mathrm{~mm} / \mathrm{yr}$ at an azimuth of $104.9 \pm 0.7^{\circ}$. The velocity of guam could be affected by strain accumulation from the subduction zone; thus the subduction rate estimate is a lower limit. However, seismic coupling in this region is believed to be low owing to the old age of the subducting plate [Uyeda and Kanamori, 1979].

[55] A similar analysis, and with the same caveats, can be applied to the western margin of the Philippine plate along the central Ryukyu arc, comparing Philippine and South China angular velocities with site oknw. The subduction rate of the Philippine plate in the central Okinawa Trough is defined by oknw's velocity relative to the Philippine plate, $98.1 \pm 1.3 \mathrm{~mm} / \mathrm{yr}$ at $131.9 \pm 0.8^{\circ}$. We predict a rate of spreading across the back arc basin at oknw of $21.4 \pm 1.4 \mathrm{~mm} / \mathrm{yr}$ at $173.7 \pm 4.1^{\circ}$ using our South China angular velocity. This is consistent with estimates of extension from seismic reflection data, 10-20 mm/yr [Park et al., 1998], and earthquake slip vectors azimuths $\left(\sim 150^{\circ}\right)$ in the central part of the Okinawa Trough [Fabbri and Fournier, 1999].

4.3.13. Somalia. [56] For the Somalia (East Africa) plate we have only two sites (MALI and SEY*) (Figure 17). SEY* is site SEY1 omitting the first year of data that is particularly noisy (Table 1) (this is the only example in this study where data were excluded). The resulting Somalia-Nubia angular velocity predicts extension across the East African Rift in an east-southeast to west-northwest direction, at rates and azimuths that are in 
approximate agreement with geologically based estimates [Jestin et al., 1994; Chu and Gordon, 1999] (Figure 21 and Table 5). For example, at the equator on the active western rift $\left(30^{\circ} \mathrm{E}\right)$ we predict $5.5 \pm 0.4 \mathrm{~mm} / \mathrm{yr}$ of extension at an azimuth of $98.3 \pm$ $4.3^{\circ}$. At $10^{\circ} \mathrm{N}, 40^{\circ} \mathrm{E}$, we predict $6.9 \pm 0.4 \mathrm{~mm} / \mathrm{yr}$ of extension at $107.9 \pm 3.7^{\circ}$

4.3.14. South China. [57] Three sites are used to define the angular velocity of the South China block: S012, SHAO, and WUHN (Figure 5). We can estimate the relative motion of adjoining plates or blocks by determining the velocity of selected sites with respect to South China or by predicting relative velocities across faults based on our angular velocity, for comparison to geologic data. Site kunm located southwest of the South China block, across the north-south striking, left-lateral Xiaojiang fault forming the block's southwest boundary, has a velocity of $10.2 \pm$ $2.2 \mathrm{~mm} / \mathrm{yr}$ with an azimuth of $200 \pm 16^{\circ}$. This is consistent with the geologic estimate of $5 \pm 3 \mathrm{~mm} / \mathrm{yr}$ for the Xiaojiang fault [England and Molnar, 1997] and with GPS measurements of Chen et al. [2000] that suggest $\sim 10 \mathrm{~mm} / \mathrm{yr}$ motion across the fault. The eastwest striking left-lateral Qinling fault defines the northern boundary with Amurian plate. At a point on the western part of the fault $\left(34.5^{\circ} \mathrm{N}, 109^{\circ} \mathrm{E}\right)$ our Amuria-South China angular velocity predicts $4.1 \pm 1.4 \mathrm{~mm} / \mathrm{yr}$ at $260 \pm 49^{\circ}$, compatible with geologic observations [Zhang et al., 1995] showing $7.2 \pm 2.2 \mathrm{~mm} / \mathrm{yr}$ of motion. On the eastern part of the fault $\left(30^{\circ} \mathrm{N}, 116^{\circ} \mathrm{E}\right)$ we predict $3.4 \pm 2.5 \mathrm{~mm} / \mathrm{yr}$ at $278 \pm 33^{\circ}$, where Zhang et al. [1995] measure $2 \pm 1 \mathrm{~mm} / \mathrm{yr}$.

\section{Conclusions}

[58] We have derived a kinematic model for the motion of 19 lithospheric plates and continental blocks based on high-precision space geodetic data. By excluding sites that may be influenced by seismic cycle effects within the plate boundary zone as well as sites affected by glacial isostatic adjustment, we believe the plate velocity model to be representative of geologically Recent motions (last $\sim 10,000$ years) and have termed it REVEL, for Recent velocities. Our model includes a rigorous and independent estimate for GPS velocity errors, facilitating tests of plate rigidity and comparison with plate velocities for different time periods. Departures from short-term rigid plate behavior due to glacial isostatic adjustment are clearly observed for North America, Eurasia, and Antarctica. Possible departures from plate rigidity due to longerterm intraplate deformation are observed in Australia and Eurasia; the latter can be interpreted to indicate extension across the Rhine Graben. We see statistically significant differences between the velocity predictions of REVEL-2000 and those of the NUVEL-1A geologic model for about one third of tested plate pairs. PacificNorth America motion and motion of the Caribbean plate with respect to North and South America are significantly faster than NUVEL-1A, presumably reflecting systematic errors in the geologic model because the relevant rate data do not reflect the full plate rate. Many other differences appear to reflect real velocity changes over the last few million years. Arabia-Nubia and ArabiaEurasia may be slowing, perhaps in response to the collision of Arabia with Eurasia and consequent increased resistance to Arabia's northward motion. Nazca-Pacific, Nazca-Antarctica, NazcaSouth America, and South America-Nubia are slower than the 3-Myr average, part of a longer-term slowing trend dating back 25-30 Myr, possibly associated with ongoing construction of the Andes [Norabuena et al., 1999].

[59] Acknowledgments. We thank the large number of individuals and institutions in the global geodetic community who generously make high-quality space geodetic data publicly available and provide long-term archives, in particular SOPAC, CDDIS, UNAVCO, and NGS. Additional RINEX data were provided by S. Miyazaki and Y. Kikuta, GSI; T. Kato, University of Tokyo, Japan; K. Duarte, IBGE, Brazil; P. Digney and
B. Twilley, AUSLIG, Australia; M. Sato, Hydrographic Department, Japan; S. Yu and J. Ng, Academia Sinica, Taiwan; E. C. Malaimani, NGRI, India; J. Cambell, University of Bonn, Germany; M. Caissy and D. O'Kane, GSD, Canada; J. Prieto-Moris, IGN, Spain; P. Nicolon, IGN, France; J. Pinto, IPCC, Portugal; S. Wdowinski and S. Naman, University of Tel Aviv, Israel; K. Larson, and J. Wahr, University of Colorado, USA; T. Van Dam, EUCGS, Luxembourg; P. Colucci, GeoDAF, Italy; and J. Weber, Grand Valley State University, USA. We thank R. Stern for suggesting the Philippine plate study; D. Jeffferson, K. Hurst, and M. Heflin at JPL, M. Mader at NGS; and Z. Altamimi, at IERS for technical assistance and advice. F. Farina and E. Norabuena provided critical help. C. Harrison, S. Stein, S. Wdowinski, P. Molnar, R. King, $\mathrm{R}$. Dokka, and an anonymous reviewer made a number of constructive comments which greatly improved the paper. This research was supported by NASA, NSF, ONR, and the Louisiana Board of Regents. All figures were made with the Generic Mapping Tools of Wessel and Smith [1995].

\section{References}

Agnew, D. C., NLOADF: A program for computing ocean-tide loading, J. Geophys. Res., 102, 5109-5110, 1997.

Alavi, M., Tectonics of the Zagros orogenic belt of Iran: New data and interpretations, Tectonophysics, 229, 211-238, 1994.

Angermann, D., J. Klotz, and C. Reigber, Space-geodetic estimation of the Nazca-South America angular velocity, Earth Planet. Sci. Lett., 171, 329-334, 1999.

Argus, D. F., and R. G. Gordon, Pacific-North American plate motion from very long baseline interferometry, compared with motion inferred from magnetic anomalies, transform faults and earthquake slip vectors, J. Geophys. Res., 95, 17,315-17,324, 1990.

Argus, D. F., and R. G. Gordon, No-net-rotation model of current plate velocities incorporating plate motion model NUVEL-1, Geophys. Res. Lett., 18, 2039-2042, 1991

Argus, D. F., and R. G. Gordon, Tests of the rigid-plate hypothesis and bounds on intraplate deformation using geodetic data from very long baseline interferometry, J. Geophys. Res., 101, 13,555-13,572, 1996.

Argus, D. F., and M. Heflin, Plate motion and crustal deformation estimated with geodetic data from the Global Positioning System, Geophys. Res. Lett., 22, 1973-1976, 1995.

Argus, D. F., W. R. Peltier, and M. M. Watkins, Glacial isostatic adjustment observed using very long baseline interferometry and satellite laser ranging geodesy, J. Geophys. Res., 104, 29,077-29,093, 1999.

Bar-Sever, Y. E., P. Kroger, and J. Borjesson, Estimating horizontal gradients of tropospheric path delay with a single GPS receiver, J. Geophys. Res., 103, 5019-5035, 1998.

Beavan, J., et al., Crustal deformation during 1994-1998 due to oblique continental collision in the central southern Alps, New Zealand, and implications for seismic potential of the Alpine fault, J. Geophys. Res., 104, 25,233-25,255, 1999.

Beavan, J., P. Tregoning, M. Bevis, T. Kato, and C. Meertens, The motion and rigidity of the Pacific plate, J. Geophys. Res, 10.1029/2001JB000282, in press, 2002

Bibee, L. D., G. G. Shor Jr., and R. S. Lu, Inter-arc spreading in the Mariana Trough, Mar. Geol., 35, 183-197, 1980.

Blewitt, G., M. B. Heflin, F. H. Webb, U. J. Lindqwister, and R. P. Malla, Global coordinates with centimeter accuracy in the International Terrestrial Reference Frame using GPS, Geophys. Res. Lett., 19, 853-856, 1992.

Bollinger, G. A., Historical and recent seismic activity in South Carolina, Bull. Seismol. Soc. Am., 62, 846-851, 1972.

Boucher, C., Z. Altamimi, and P. Sillard, The 1997 International Terrestrial Reference Frame (ITRF-97), IERS Tech. Note, 27, Obs. de Paris, Paris, 1999.

Bouin, M.-N., and C. Vigny, New constraints on Antarctic plate motion and deformation from GPS data, J. Geophys. Res., 105, 28,279-28,293, 2000.

Calais, E., Continuous GPS measurements across the western Alps, 19961998, J. Geophys. Int., 138, 221-230, 1999.

Calais, E., and S. Amarjargal, New constraints on current deformation in Asia from continuous GPS measurements at Ulan Baatar, Mongolia, Geophys. Res. Lett., 27, 1527-1530, 2000.

Camelbeeck, T., and M. Meghraoui, Geological and geophysical evidence for large palaeo-earthquakes with surface faulting in the Roer Graben (northwest Europe), Geophys. J. Int., 132, 347-362, 1998.

Cande, S. C., and D. V. Kent, A new geomagnetic polarity timescale for the Late Cretaceous and Cenozoic, J. Geophys. Res., 97, 13,915-13,917, 1992.

Cazenave, A., J. J. Valette, and C. Boucher, Positioning results with DORIS on SPOT-2 after first year of mission, J. Geophys. Res., 97, 7109-7119, 1992.

Cazenave, A., P. Gegout, L. Soudarin, K. Dominh, F. Barlier, P. Exertier, 
and Y. Boudon, Geodetic results from Lageos 1 and DORIS satellite data, in Contributions of Space Geodesy and Geodynamics: Crustal Dynamics, Geodyn. Ser., vol. 23, edited by D. E. Smith and D. L. Turcotte, pp. 81-98, AGU, Washington, D. C., 1993.

Chamot-Rooke, N., and X. Le Pichon, GPS determined eastward Sundaland motion with respect to Eurasia confirmed by earthquakes slip vectors at Sunda and Philippine trenches, Earth Planet. Sci. Lett., 173, 439-455, 1999.

Chapman, M. E., and S. C. Solomon, North American-Eurasian plate boundary in northeast Asia, J. Geophys. Res., 81, 921-930, 1976.

Chase, C. G., The N plate problem of plate tectonics, Geophys. J. R. Astron. Soc., 29, 117-122, 1972 .

Chase, C. G., Plate kinematics: The Americas, East Africa, and the rest of the world, Earth Planet. Sci. Lett., 37, 353-368, 1978.

Chen, Z., B. C. Burchfiel, Y. Liu, R. W. King, L. H. Royden, W. Tang, E. Wang, J. Zhao, and X. Zhang, Global Positioning System measurements from eastern Tibet and their implications for India/Eurasia intercontinental deformation, J. Geophys. Res., 105, 16,215-16,227, 2000.

Chu, D., and R. G. Gordon, Current plate motions across the Red Sea, Geophys. J. Int., 135, 313-328, 1998.

$\mathrm{Chu}$, D., and R. G. Gordon, Evidence for motion between Nubia and Somalia along the Southwest Indian Ridge, Nature, 398, 64-67, 1999.

Coates, R. J., H. Frey, G. D. Mead, and J. M. Bosworth, Space age geodesy: The NASA crustal dynamics project, IEEE Trans. Geosci. Remote Sens., GE-23, 360-368, 1985.

Conder, J. A., and D. W. Forsyth, Do the 1998 Antarctic plate earthquake and its aftershocks delineate a plate boundary, Geophys. Res. Lett., 27, 2309-2312, 2000.

Conder, J. A., and D. W. Forsyth, Seafloor spreading on the Southeast Indian Ridge over the last one million years: A test of the Capricorn plate hypothesis, Earth Planet. Sci. Lett., 188, 91-105, 2001.

Cook, D. B., K. Fujita, and C. A. McMullen, Present-day interactions in northeast Asia: North American, Eurasian, and Okhotsk plates, J. Geodyn., 6, 33-51, 1986.

Cretaux, J. F., L. Soudarin, A. Cazenave, and F. Bouille, Present-day tectonic plate motions and crustal deformations from the DORIS space system, J. Geophys. Res., 103, 30,167-30,181, 1998.

Davis, J. L., J. X. Mitrovica, H.-G. Scherneck, and H. Fan, Investigation of Fennoscandian glacial isostatic adjustment using modern sea level records, J. Geophys. Res., 104, 2733-2747, 1999.

Delvaux, D., R. Moeys, G. Stapel, C. Petit, K. Levi, A. Miroshnichenko, V. Ruzhich, and V. San'kov, Paleostress reconstructions and geodynamics of the Baikal region, central Asia, part 2, Cenozoic rifting, Tectonophysics, 282, 1-38, 1997.

DeMets, C., A reappraisal of seafloor spreading lineations in the Gulf of California: Implications for the transfer of Baja California to the Pacific plate and estimates of Pacific-North American motion, Geophys. Res. Lett., 22, 3545-3548, 1995.

DeMets, C., and T. H. Dixon, New kinematic models for Pacific-North America motion from $3 \mathrm{Ma}$ to present, 1, Evidence for steady motion and biases in the NUVEL-1A model, Geophys. Res. Lett., 26, $1921-$ 1924, 1999

DeMets, C., R. G. Gordon, and D. F. Argus, Intraplate deformation and closure of the Australia-Antarctica-Africa plate circuit, J. Geophys. Res., 93, 11,877-11,897, 1988.

DeMets, C., R. G. Gordon, D. F. Argus, and S. Stein, Current plate motions, Geophys. J. Int., 101, 425-478, 1990.

DeMets, C., R. G. Gordon, D. F. Argus, and S. Stein, Effect of recent revisions to the geomagnetic time scale on estimates of current plate motion, Geophys. Res. Lett., 21, 2191-2194, 1994.

DeMets, C., P. Jansma, G. Mattioli, T. Dixon, F. Farina, R. Bilham, E. Calais, and P. Mann, GPS geodetic constraints on Caribbean-North America plate motion, Geophys. Res. Lett., 27, 437-440, 2000.

Deng, J., and L. R. Sykes, Determination of Euler pole for contemporary relative motion of Caribbean and North American plates using slip vectors of interplate earthquakes, Tectonics, 14, 39-53, 1995.

Dixon, T. H., An introduction to the Global Positioning System and some geological applications, Rev. Geophys., 29, 249-276, 1991.

Dixon, T. H., GPS measurement of strain accumulation across the middle America trench and relative motion of the Cocos and Caribbean plates, Geophys. Res. Lett., 20, 2167-2179, 1993.

Dixon, T. H., and A. Mao, A GPS estimate of relative motion between North and South America, Geophys. Res. Lett., 24, 535-538, 1997.

Dixon, T. H., G. Gonzalez, S. M. Lichten, D. M. Tralli, G. Ness, and P. Dauphin, A preliminary determination of Pacific-North America relative motion in the southern Gulf of California using the Global Positioning System, Geophys. Res. Lett., 18, 861-864, 1991a.

Dixon, T. H., G. Gonzalez, S. Lichten, and E. Katsigris, First epoch geodetic measurements with the Global Positioning System across the northern Caribbean plate boundary zone, J. Geophys. Res., 96, 2397-2415, 1991 b.
Dixon, T. H., M. Bursik, S. K. Wolfe, M. Heflin, F. Webb, F. Farina, and S. Robaudo, Constraints on deformation of the resurgent dome, Long Valley Caldera from space geodesy, in Contributions of Space Geodesy and Geodynamics: Crustal Dynamics, Geodyn. Ser., vol. 23, edited by D. E. Smith and D. L. Turcotte, pp. 193-213, AGU, Washington, D. C., 1993.

Dixon, T. H., A. Mao, and S. Stein, How rigid is the stable interior of the North American plate, Geophys. Res. Lett., 23, 3035-3038, 1996.

Dixon, T. H., A. Mao, M. Bursik, M. Heflin, J. Langbein, R. Stein, and F. Webb, Continuous monitoring of surface deformation at Long Valley Caldera with GPS, J. Geophys. Res., 102, 12,017-12,034, 1997.

Dixon, T. H., F. Farina, C. DeMets, P. Jansma, P. Mann, and E. Calais, Relative motion between the Caribbean and North American plates based on a decade of GPS observations, J. Geophys. Res., 103, 15,157-15,182, 1998.

Dixon, T. H., M. Miller, F. Farina, H. Wang, and D. Johnson, Present-day motion of the Sierra Nevada block, and some tectonic implications for the Basin and Range province, North American Cordillera, Tectonics, 19, $1-24,2000$ a.

Dixon, T. H., C. DeMets, F. Suarez-Vidal, J. Fletcher, B. Marquez-Azua, M. Miller, O. Sanchez, and P. Umhoefer, New kinematic models for Pacific-North America motion from $3 \mathrm{Ma}$ to Present, II, Evidence for a Baja California shear zone, Geophys. Res. Lett., 27, 3961-3964, 2000b.

Doser, D. I., Faulting within the western Baikal rift as characterized by earthquake studies, Tectonophysics, 196, 87-107, 1991.

England, P., and P. Molnar, The field of crustal velocity in Asia calculated from Quaternary rates of slip on faults, Geophys. J. Int., 130, 551-582, 1997.

Fabbri, O., and M. Fournier, Extension in the southern Ryukyu arc (Japan): Link with oblique subduction and back arc rifting, Tectonics, 18, 486497, 1999

Gordon, R. G., The plate tectonic approximation: plate non-rigidity, diffuse plate boundaries, and global plate reconstructions, Annu. Rev. Earth Planet. Sci., 26, 615-642, 1998.

Gordon, R. G., C. DeMets, and J.-Y. Royer, Evidence for long-term diffuse deformation of the lithosphere of the equatorial Indian Ocean, Nature, 395, 370-374, 1998.

Gordon, R. G., D. F. Argus, and M. B. Heflin, Revised estimate of the angular velocity of India relative to Eurasia, Eos Trans. AGU, 80(46), Fall Meet. Suppl., F273, 1999.

Grenerczy, G., A. Kenyeres, and I. Fejes, Present crustal movement and strain distribution in central Europe inferred from GPS measurements, J. Geophys. Res., 105, 21,835-21,846, 2000.

Heflin, M., et al., Global geodesy using GPS without fiducial sites, Geophys. Res. Lett., 19, 131-134, 1992.

Heki, K., S. Miyazaki, H. Takahashi, M. Kasahara, F. Kimata, S. Miura, N. F. Vasilenko, A. Ivashenko, and K.-D. An, The Amurian plate motion and current plate kinematics, J. Geophys. Res., 104, 29,147-29,155, 1999.

Herraiz, M., et al., The recent (upper Miocene to Quaternary) and present tectonics stress distributions in the Iberian Peninsula, Tectonics, 19, $762-786,2000$

Hillis, R. R., and S. D. Reynolds, The Australian stress map, J. Geol. Soc. London, 157, 915-921, 2000.

Holt, W. E., N. Chamot-Rooke, X. Le Pichon, A. J. Haines, B. Shen-Tu, and J. Ren, Velocity field in Asia inferred from Quaternary fault slip rates and Global Positioning System observations, J. Geophys. Res., 105, $19,185-19,209,2000$

Hreinsdottir, S., P. Einarsson, and F. Sigmundsson, Crustal deformation at the oblique spreading Reykjanes Peninsula, SW Iceland: GPS measurements from 1993 to 1998, J. Geophys. Res., 106, 13,803-13,816, 2001.

Hussong, D. M., and S. Uyeda, Tectonic processes and the history of the Mariana Arc, A synthesis of the results of Deep Sea Drilling Project Leg 60, Initial Rep. Deep Sea Drill. Proj., 60, 909-929, 1981.

Ishihara, T., R. J. Stern, P. Fryer, S. Bloomer, and N. C. Becker, Seafloor spreading in the southern Mariana Trough inferred from 3-componenet magnetometer data, Eos Trans. $A G U$, 82(47), Fall Meet. Suppl., Abstract T41C-0895, 2001.

Izzeldin, A. Y., Transverse structures in the central part of the Red Sea and implications on early stages of oceanic accretion, Geophys. J., 96, 117-129, 1989.

Jansma, P. E., G. S. Mattioli, A. Lopez, C. DeMets, T. H. Dixon, P. Mann, and E. Calais, Neotectonics of Puerto Rico and the Virgin Islands, northeastern Caribbean, from GPS geodesy, Tectonics, 19, 1021-1037, 2000.

Jestin, F., P. Huchon, and J. M. Gaulier, The Somalia plate and the East African Rift System: Present-day kinematics, Geophys. J. Int., 116, 637-654, 1994.

Jonsson, S., P. Einarsson, and F. Sigmundsson, Extension across a divergent plate boundary, the Eastern Volcanic Rift Zone, south Iceland, 1967-1994, observed with GPS and electronic distance measurements, J. Geophys. Res., 102, 11,913-11,929, 1997. 
Jordan, T. H., The present-day motions of the Caribbean plate, J. Geophys. Res., 80, 4433-4439, 1975.

Kato, T., Y. Kotake, T. Chacin, Y. Iimuta, S. Miyazaki, T. Kanazawa, and K. Suyehiro, An estimate of the Philippine Sea plate motion derived from the Global Position System observation at Okino Torishima Japan, J. Geod. Soc. Jpn., 42, 233-243, 1996.

Kato, T., et al., Initial results from WING, the continuous GPS network in the western Pacific area, Geophys. Res. Lett., 25, 369-372, 1998.

Klepeis, K. A., and L. A. Lawver, Tectonics of the Antarctic-Scotia plate boundary near Elephant and Clarence Islands, West Antarctica, J. Geophys. Res., 101, 20,211-20,231, 1996.

Klinger, Y., J. P. Avouac, N. Abou Karaki, L. Dorbath, D. Bourles, and J. L. Reyss, Slip rate on the Dead Sea transform fault in northern Araba valley (Jordan), Geophys. J. Int., 142, 755-768, 2000.

Kogan, M. G., G. M. Steblov, R. W. King, T. A. Herring, D. I. Frolov, S. G. Egorov, V. Y. Levin, A. Lerner-Lam, and A. Jones, Geodetic constraints on the rigidity and relative motion of Eurasia and North America, Geophys. Res. Lett., 27, 2041-2044, 2000.

Kotake, Y., T. Kato, S. Miyazaki, and A. Sengoku, Relative motion of the Philippine Sea plate derived from GPS observations and tectonics of south-western Japan, J. Seismol. Soc. Jpn., 51, 171-180, 1998.

Kreemer, C., J. Haines, W. E. Holt, G. Blewitt, and D. Lavalee, On the determination of a global strain rate model, Earth Planets Space, 52, $765-770,2000$

Langbein, J., and H. Johnson, Correlated errors in geodetic time series: Implications for time-dependent deformation, J. Geophys. Res., 102, 591-603, 1997 .

Larson, K. M., J. T. Freymueller, and S. Philipsen, Global plate velocities from the Global Positioning System, J. Geophys. Res., 102, 9961-9981, 1997.

Leroy, S., A. Mauffret, P. Patriat, and B. Mercier de Lepinay, An alternative interpretation of the Cayman trough evolution from a re-identification of magnetic anomalies, Geophys. J. Int., 141, 539-557, 2000.

Macdonald, K. C., and T. L. Holcombe, Inversion of magnetic anomalies and sea-floor spreading in the Cayman Trough, Earth Planet. Sci. Lett. 40, 407-414, 1978

Mader, G. L., GPS antenna calibration at the National Geodetic Survey, GPS Solutions, 3, 50-58, 1999.

Malaimani, E. C., J. Campbell, B. Gorres, H. Kotthoff, and S. Smaritschnik Indian plate kinematic studies by GPS-geodesy, Earth Planets Space, 52 $741-745,2000$

Mann, P., F. W. Taylor, R. Edwards, and T. L. Ku, Actively evolving microplate formation by oblique collision and sideways motion along strike-slip faults: An example from the northeastern Caribbean plate margin, Tectonophysics, 246, 1-69, 1995.

Mao, A., Geophysical applications of the Global Positioning System, dissertation, Univ. of Miami, Coral Gables, Fla., 1998.

Mao, A., C. G. A. Harrison, and T. H. Dixon, Noise in GPS coordinate time series, J. Geophys. Res., 104, 2797-2816, 1999.

McClusky, S., et al., Global Positioning System constraints on plate kinematics and dynamics in the eastern Mediterranean and Caucasus, J. Geophys. Res., 105, 5695-5719, 2000

Meghraoui, M., T. Camelbeeck, K. Vanneste, M. Brondeel, and D. Jongmans, Active faulting and paleoseismology along the Bree fault, lower Rhine graben, Belgium, J. Geophys. Res., 105, 13,809-13,841, 2000.

Minster, J. B., and T. H. Jordan, Present-day plate motions, J. Geophys. Res., 83, 5331-5354, 1978.

Minster, J. B., T. H. Jordan, P. Molnar, and E. Haines, Numerical modeling of instantaneous plate tectonics, Geophys. J. R. Astron. Soc., 36, $541-$ $576,1974$.

Mitrovica, J. X., A. M. Forte, and M. Simons, A reappraisal of postglacial decay times from Richmond Gulf and James Bay, Canada, Geophys. J. Int., 142, 783-800, 2000.

Newman, A., S. Stein, J. Weber, J. Engeln, A. Mao, and T. Dixon, Slow deformation and lower seismic hazard at the New Madrid seismic zone, Science, 284, 619-621, 1999.

Niell, A. E., Global mapping functions for the atmosphere delay at radio wavelengths, J. Geophys. Res., 101, 3227-3246, 1996

Nocquet, J.-M., E. Calais, Z. Altamimi, P. Sillard, and C. Boucher, Intraplate deformation in western Europe deduced from an analysis of the International Terrestrial Reference Frame 1997 (ITRF97) velocity field, J. Geophys. Res., 106, 11,239-11,258, 2001.

Norabuena, E. O., L. Leffler-Griffin, A. Mao, T. H. Dixon, S. Stein, I. S. Sacks, L. Ocola, and M. Ellis, Space geodetic observations of NazcaSouth American convergence across the central Andes, Science, 279 $358-362,1998$

Norabuena, E. O., T. H. Dixon, S. Stein, and C. G. A. Harrison, Decelerating Nazca-South America and Nazca-Pacific plate motions, Geophys. Res. Lett., 26, 3405-3408, 1999.

Nuttli, O. W., The Mississippi Valley earthquakes of 1811-1812: Intensi- ties, ground motions, and magnitudes, Bull. Seismol. Soc. Am., 63, 227-248, 1973

Park, J.-O., H. Tokyama, M. Shinohara, K. Suyehiro, and A. Taira, Seismic record of tectonics evolution and backarc rifting in the southern Ryukyu island arc system, Tectonophysics, 294, 21-42, 1998

Paul, J., et al., Microstrain stability of Peninsular India 1864-1994, Proc. Indian Acad. Sci., 104, 131-146, 1995.

Paul, J., et al., The motion and active deformation of India, Geophys. Res. Lett., 28, 647-650, 2001.

Pe'eri, S., S. Wdowinski, A. Shtibelman, N. Bechor, Y. Bock, R. Nikolaidis, and M. Van Domselaar, Current plate motion across the Dead Sea Fault from three years of continuous GPS monitoring, Geophys. Res. Lett, 10.1029/2001GL013879, in press, 2002.

Pelayo, A. M., and D. A. Wiens, Seismotectonics and relative plate motions in the Scotia Sea region, J. Geophys. Res., 94, 7293-7320, 1989.

Peltier, W. R., Ice age paleotopography, Science, 265, 195-201, 1994.

Peltier, W. R., Postglacial variations in the level of the sea: Implications for climate dynamics and solid-earth geophysics, Rev. Geophys., 36, 603-689, 1998a.

Peltier, W. R., A space geodetic target for mantle viscosity discrimination: Horizontal motions induced by glacial isostatic adjustment, Geophys. Res. Lett., 25, 543-546, 1998b.

Peltzer, G., and F. Saucier, Present-day kinematics of Asia derived from geologic fault rates, J. Geophys. Res., 101, 27,943-37,956, 1996.

Perez, O. M., R. Bilham, R. Bendick, J. R. velandia, N. Hernandez, C. Moncayo, M. Hoyer, and M. Kozuch, Velocity field across the southern Caribbean plate boundary and estimates of Caribbean/South-American plate motion using GPS geodesy 1994-2000, Geophys. Res. Lett., 28, 2987-2990, 2001

Plenefisch, T., and K.-P. Bonjer, The stress field in the Rhine Graben area inferred from earthquake focal mechanisms and estimation of frictional parameters, Tectonophysics, 275, 71-97, 1997.

Pollitz, F., Postseismic relaxation theory on the spherical earth, Bull. Seismol. Soc. Am., 82, 422-453, 1992.

Pollitz, F. F., and T. H. Dixon, GPS measurements across the northern Caribbean plate boundary: impact of postseismic relaxation following historic earthquakes, Geophys. Res. Lett., 25, 2233-2236, 1998.

Pollitz, F., and S. Sacks, Fault model of the 1891 Nobi earthquake from historic triangulation and leveling, J. Phys. Earth, 42, 1-43, 1994.

Pubellier, M., A. Mauffret, S. Leroy, J. M. Vila, and H. Amilcar, Plate boundary readjustment in oblique convergence: Example of the Neogene of Hispaniola, Greater Antilles, Tectonics, 19, 630-648, 2000.

Rangin, C., X. Le Pichon, S. Mazzotti, M. Pubellier, N. Chamot-Rooke, M. Aurelio, A. Walpersdorf, and R. Quebral, Plate convergence measured by GPS across the Sundaland/Philippine Sea plate deformed boundary: The Philippines and eastern Indonesia, Geophys. J. Int., 139, 296-316, 1999.

Reilinger, R., S. C. McClusky, M. G. Oral, R. W. King, M. N. Tosoz, A. A. Barka, I. Kinkik, O. Lenk, and I. Sani, Global Positioning System measurements of present-day crustal movements in the Arabia-Africa-Eurasia plate collision zone, J. Geophys. Res., 102, 9983-9999, 1997.

Ribeiro, A., J. Cabral, R. Baptista, and L. Matias, Stress pattern in Portugal mainland and the adjacent Atlantic region, West Iberia, Tectonics, 15 , $641-659,1996$.

Robaudo, S., and C. G. A. Harrison, Plate tectonics from SLR and VLBI data, in Contributions of Space Geodesy and Geodynamics: Crustal Dynamics, Geodyn. Ser., vol. 23, edited by D. E. Smith and D. L. Turcotte, pp. 51-71, AGU, Washington, D. C., 1993.

Robbins, J. W., D. E. Smith, and C. Ma, Horizontal crustal deformation and large scale plate motions inferred from space geodetic techniques, in Contributions of Space Geodesy and Geodynamics: Crustal Dynamics, Geodyn. Ser., vol. 23, edited by D. E. Smithand D. L. Turcotte, pp. 21-36, AGU, Washington, D. C., 1993.

Rosencrantz, E., and P. Mann, SeaMarc-II mapping of transform faults in the Cayman Trough, Caribbean Sea, Geology, 19, 690-693, 1991.

Rosencrantz, E., M. I. Ross, and J. G. Sclater, Age and spreading history of the Cayman Trough as determined from depth, heat flow and magnetic anomalies, J. Geophys. Res., 93, 2141-2157, 1988.

Royer, J.-Y., and R. G. Gordon, The motion and boundary between the Capricorn and Australian plates, Science, 277, 1268-1274, 1997.

Ryan, J. W., T. A. Clark, C. Ma, D. Gordon, D. S. Caprette, and W. E. Himwich, Global scale tectonic plate motions measured with CDP VLBI data, in Contributions of Space Geodesy and Geodynamics, Crustal Dynamics, Geodyn. Ser, vol. 23, edited by D. E. Smith and D. L. Turcotte, pp. 37-49, AGU, Washington, D. C., 1993.

Sato, K., Tectonic plate motion and deformation inferred from very long baseline interferometry, Tectonophysics, 220, 69-87, 1993.

Schweig, E. S., and M. A. Ellis, Reconciling short recurrence intervals with minor deformation in the New Madrid seismic zone, Science, 264, $1308-1311,1994$ 
Schwiderski, E. W., On charting ocean tides, Rev. Geophys., 18, 243-268, 1980.

Sengoku, A., A plate motion study using Ajisai SLR data, Earth Planets Space, 50, 611-627, 1998.

Seno, T., S. Stein, and A. E. Gripp, A model for the motion of the Philippine Sea plate consistent with NUVEL-1A and geological data, J. Geophys. Res., 98, 17,941-17,948, 1993.

Seno, T., T. Sakurai, and S. Stein, Can the Okhotsk plate be discriminated from the North American plate?, J. Geophys. Res., 101, 11,305-11,315, 1996.

Shen, Z.-K., C. Zhao, A. Yin, Y. Li, D. D. Jackson, P. Fang, and D. Dong, Contemporary crustal deformation in east Asia constrained by Global Positioning System measurements, J. Geophys. Res., 105, 5721-5734, 2000.

Sigmundsson, F., P. Einarsson, R. Bilham, and E. Sturkell, Rift-transform kinematics in south Iceland: Deformation from Global Positioning System measurements, 1986 to 1992, J. Geophys. Res., 100, 6235-6248, 1995.

Sillard, P., Z. Altamimi, and C. Boucher, The ITRF96 realization and its associated velocity field, Geophys. Res. Lett., 25, 3222-3226, 1998.

Smith, D., R. Kolenkiewicz, P. J. Dunn, J. W. Robbins, M. H. Torrence, S. Klosko, R. G. Williamson, E. C. Pavlis, N. B. Douglas, and S. K. Fricke, Tectonic motion and deformation from satellite laser ranging to LAGEOS, J. Geophys. Res., 95, 22,013-22,041, 1990.

Somoza, R., Updated Nazca (Farallon)-South America relative motions during the last $40 \mathrm{My}$ : Implications for mountain building in the central Andean region, J. S. Am. Earth Sci., 11, 211-215, 1998.

Sorlien, C. C., M. Kamerling, and D. Mayerson, Block rotation and termination of the Hosgri strike slip fault, California, from three-dimensional map restoration, Geology, 27, 1039-1042, 1999.

Soudarin, L., and A. Cazenave, Global geodesy using DORIS data on SPOT-2, Geophys. Res. Lett., 20, 289-292, 1993.

Soudarin, L., and A. Cazenave, Large-scale tectonics plate motions measured with the DORIS space geodesy system, Geophys. Res. Lett, 22, 469-472, 1995.

Spitzak, S., and C. DeMets, Constraints on present-day plate motions south of $30^{\circ} \mathrm{S}$ from satellite altimetry, Tectonophysics, 253, 167-208, 1996.

Stein, S., and R. G. Gordon, Statistical tests of additional plate boundaries from plate motion inversions, Earth Planet. Sci. Lett., 69, 401-412, 1984.

Stein, S., C. DeMets, R. G. Gordon, J. Brodhold, D. Argus, J. F. Engeln, P. Lundgren, C. Stein, D. Wiens, and D. Woods, A test of alternative Caribbean plate relative motion models, J. Geophys. Res., 93, 30413050, 1988.

Stern, R. J., N. C. Smoot, and M. Rubin, Unzipping of the volcano arc, Japan, Tectonophysics, 102, 153-174, 1984.

Sutherland, R., The Australian-Pacific boundary and Cenozoic plate motions in the SW Pacific: Some constraints from Geosat data, Tectonics, 14, 819-831, 1995.

Sutherland, R., F. Davey, and J. Beavan, Plate boundary deformation in South Island, New Zealand, is related to inherited lithospheric structure, Earth Planet. Sci. Lett., 177, 141-151, 2000.

Sykes, L. R., W. R. McCann, and A. L. Kafka, Motion of the Caribbean plate during the last 7 million years and implications for earlier Cenozoic movements, J. Geophys. Res., 87, 10,656-10,676, 1982.
Tebbens, S. F., and S. C. Cande, Southeast Pacific tectonic evolution from early Oligocene to Present, J. Geophys. Res., 102, 12,061-12,084, 1997.

Uyeda, S., and H. Kanamori, Back-arc opening and the mode of subduction, J. Geophys. Res., 84, 1049-1061, 1979.

van Vliet-Lanoe, B., S. Bonnet, B. Hallegouet, and M. Laurent, Neotectonics and seismic activity in the Armorican and Cornubian massifs: Regional stress field with glacio-isostatic influence?, J. Geodyn., 24, 219-239, 1997.

Walpersdorf, A., C. Vigny, P. Manurung, C. Subarya, and S. Sutisna, Determining the Sula block kinematics in the triple junction area in Indonesia by GPS, Geophys. J. Int., 135, 351-361, 1998.

Ward, S. N., Pacific-North America plate motions: New results from very long baseline interferometry, J. Geophys. Res., 95, 21,965-21,981, 1990.

Weber, J., S. Stein, and J. Engeln, Estimation of intraplate strain accumulation in the New Madrid seismic zone from repeat GPS surveys, Tectonics, 17, 250-266, 1998.

Weber, J., et al., A GPS estimate of relative motion between the Caribbean and South American plates, and geologic implications for Trinidad and Venezuela, Geology, 29, 75-78, 2001.

Wei, D., and T. Seno, Determination of the Amurian plate motion, in Mantle Dynamics and Plate Interactions in East Asia, Geodyn. Ser., vol. 27, edited by M. F. J. Flower et al., pp. 337-346, AGU, Washington, D. C., 1998 .

Wessel, P., and W. H. F. Smith, New version of generic mapping tools released, Eos Trans. $A G U, 76,329,1995$.

Wiens, D. A., et al., A diffuse plate boundary model for Indian Ocean tectonics, Geophys. Res. Lett., 12, 429-432, 1985.

Yu, S.-B., H.-Y. Chen, and L.-C. Kuo, Velocity field of GPS stations in the Taiwan area, Tectonophysics, 274, 41-59, 1997.

Yu, S.-B., L.-C. Chen, R. S. Punongbayan, and E. G. Ramos, GPS observation of crustal deformation in the Taiwan-Luzon region, Geophys. Res. Lett., 26, 923-926, 1999.

Zhang, J., Y. Bock, H. Johnson, P. Fang, S. Williams, J. Genrich, S. Wdowinski, and J. Bahr, Southern California permanent GPS geodetic array: Error analysis of daily position estimates and site velocities, J. Geophys, Res., 102, 18,035-18,055, 1997.

Zhang, Y. Q., J. L. Mercier, and P. Vergely, Extension in the graben systems around the Ordos (China), and its contribution to the extrusion tectonics of south China with respect to Gobi-Mongolia, Tectonophysics, 285, $21-41,1995$.

Zonenshain, L. P., and L. A. Savostin, Geodynamics of the Baikal rift zone and plate tectonics of Asia, Tectonophysics, 76, 1-45, 1981.

Zumberge, J. F., M. Heflin, D. Jefferson, M. Watkins, and F. Webb, Precise point positioning for efficient analysis of GPS data, J. Geophys. Res., 102, 5005-5017, 1997

T. H. Dixon and A. Mao, Rosenstiel School of Marine and Atmospheric Science, University of Miami, 4600 Rickenbacker Causeway, Miami, FL 33149, USA. (tdixon@rsmas.miami.edu; amao@rsmas.miami.edu)

G. F. Sella, Department of Geological Sciences, Northwestern University, 1847 Sheridan Rd, Evanston, IL 60208, USA. (sella@earth.northwestern. edu) 


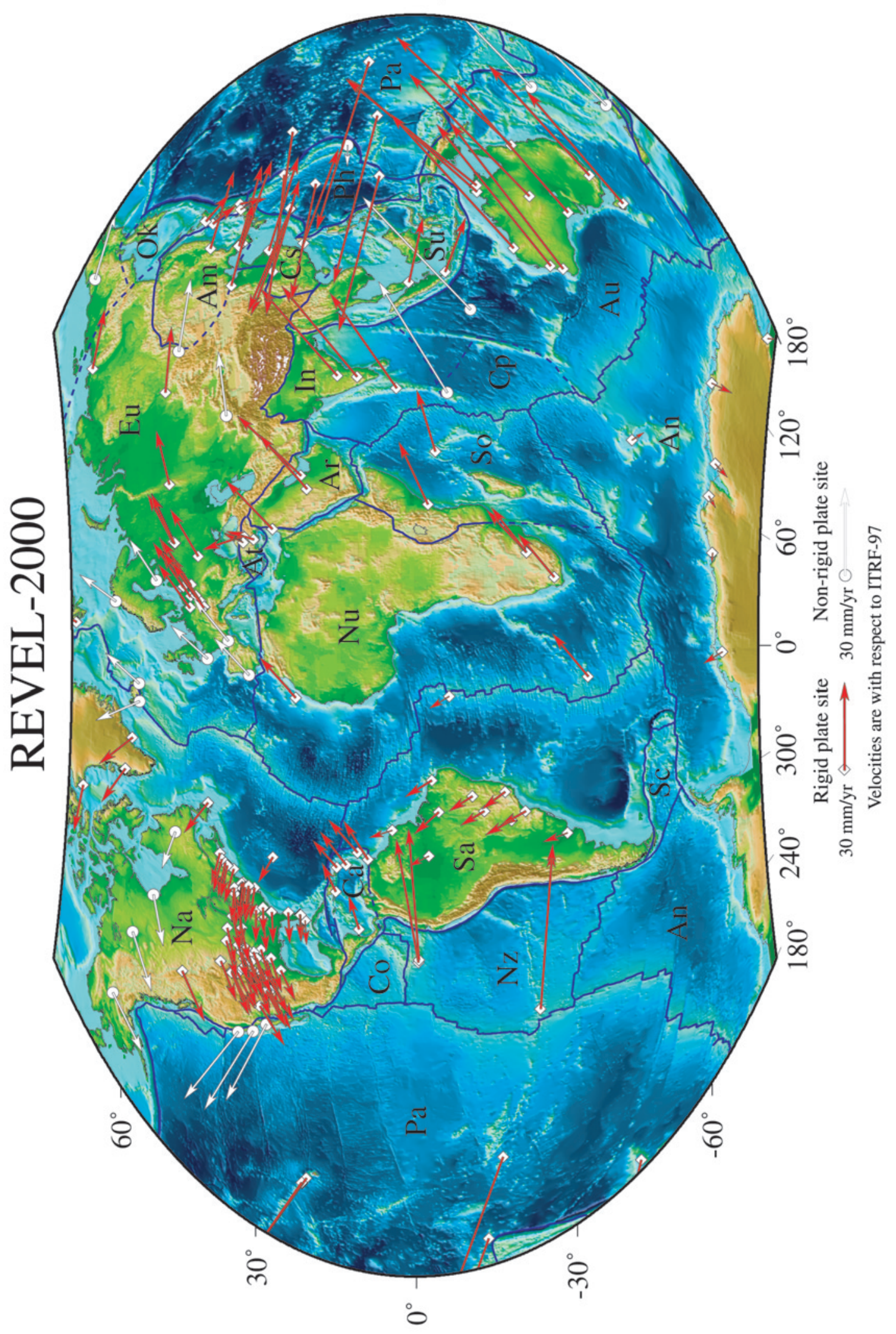

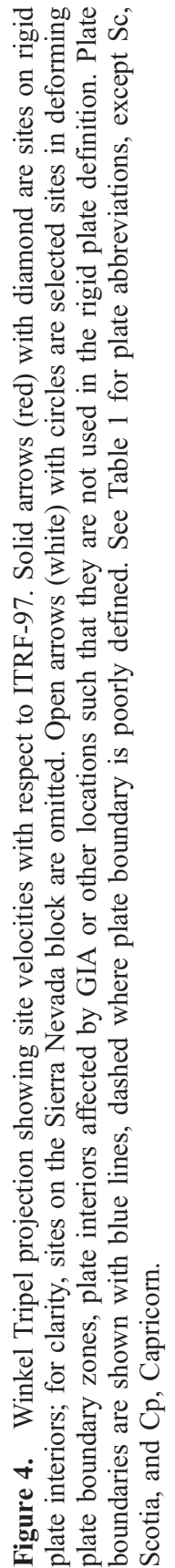

